\title{
DEEPLY-TOWED UNDERWATER VEHICLE SYSTEMS: A Verified Analytical Procedure for Creating Parameterized Dynamic Models
}

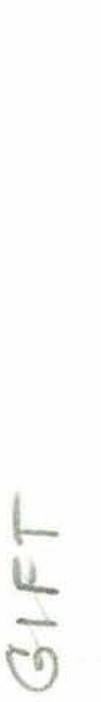

\author{
by \\ Franz S. Hover \\ B.S.M.E., Ohio Northern University (1987) \\ Submitted to the \\ Massachusetts Institute of Technology/ \\ Woods Hole Oceanographic Institution \\ Joint Program in Oceanography and Oceanographic Engineering \\ in Partial Fulfillment of the Requirements \\ for the Degree of \\ Master of Science \\ at the \\ Massachusetts Institute of Technology \\ and the \\ Woods Hole Oceanographic Institution
}

\begin{tabular}{|c|}
\hline MARINE \\
BIOLOGICAL \\
LABORATORY \\
\hline LIBRARY \\
\hline $\begin{array}{c}\text { WOODS HOLE, MASS. } \\
\text { W. H. O. I. }\end{array}$ \\
\hline
\end{tabular}

August 1989

Copyright (c) Franz S. Hover 1989

The author hereby grants to MIT permission to reproduce and to distribute copies of this thesis document in whole or in part.

Signature of Author

Joint Program in Oceanography and Oceanographic Engineering Massachusetts Institute of Technology/ Woods Hole Oceanographic Institution

Certified by

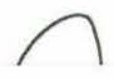

$\frown$ 


\title{
DEEPLY-TOWED UNDERWATER VEHICLE SYSTEMS: A Verified Analytical Procedure for Creating Parameterized Dynamic Models
}

\author{
by \\ Franz S. Hover \\ Submitted to the Massachusetts Institute of Technology/ \\ Woods Hole Oceanographic Institution \\ Joint Program in Oceanographic Engineering \\ on August 11, 1989, in partial fulfillment of the \\ requirements for the degree of \\ Master of Science
}

\begin{abstract}
The dynamics of deeply-towed cable/vehicle systems are governed by nonlinear partial differential equations and as a result, trajectory control is generally difficult using the available techniques. This work examines the possibility of utilizing parametric dynamic models in differential equation form, to present a far more tractable controls problem. A learning-model method for generating accurate approximations of this type is used, and the identification process is unique in that an analytically-based model provides the primary data sets, allowing for a priori characterization of system responses without using any real data. The performances of the parametric forms are then verified through comparison of model output against actual sea data obtained during recent cruises in the Caribbean and Mediterranean Seas. The respective merits and limitations of several different model structures are discussed, with respect to both pure performance and identification efficiency.
\end{abstract}

Thesis Supervisor: Dr. Dana R. Yoerger

Title: Associate Scientist, Woods Hole Oceanographic Institution 


\section{Dedication}

I will probably always dedicate the important things to my parents.

\section{Acknowledgements}

I thank the following persons for their many contributions to this work: Dana Yoerger, Mike Triantafyllou, Mark Grosenbaugh, Mike Drooker at DESLAB, and other lab members at WHOI and at MIT. My closest friends (you know who you are) were responsible for my sanity during this time.

The Office of Naval Research is gratefully acknowledged for its financial support of my graduate education, under Contract N00014-85-G-0084; in addition, this work has been sponsored in part by the National Science Foundation under Contract OCE-8511431. Finally, the International Business Machines Corporation is acknowledged for graciously providing the computing facilities that were critical to this thesis. 


\section{Table of Contents}

Abstract $\quad 2$

Dedication $\quad 4$

Table of Contents $\quad 5$

List of Figures $\quad 7$

List of Tables $\quad 9$

1. Introduction $\quad 10$

1.1 Motivation 10

$\begin{array}{ll}1.2 \text { Outline of the Thesis } & 13\end{array}$

2. Analytical Model Derivation $\quad 15$

2.1 Introduction $\quad 15$

2.2 Equations of Motion and Statics $\quad 15$

2.3 Compatibility Relations 19

2.4 Solving the Modified Wave Equation 23

2.5 Vehicle Dynamics … 31

2.6 Implementation Notes $\quad 34$

2.6.1 Statics 34

2.6.2 Solving the Dynamics $\quad 34$

2.6.3 Forcing Functions $\quad 35$

$\begin{array}{ll}2.7 \text { Appendix } & 36\end{array}$

2.7.1 Effective Tension $\quad 36$

2.7.2 Propagating the Euler Angles 36

3. Verification of the Analytical Model 39

3.1 AUTEC Range Run 39

3.2 Tyrrhenian Sea Data $\quad 44$

3.3 Conclusions 48

4. Observations on Cable Behavior 49

4.1 Time Delay 49

4.2 Settling Time and Optimized Ship Motions 53

5. The Identification Method 57

5.1 Analytical Model Use in Identification $\quad 57$

5.2 Model Identification Method $\quad 59$

5.2.1 Learning Model Method 60

5.2.2 Nelder-Meade Simplex Algorithm 61

6. Model Forms and Results $\quad 64$

6.1 Ship Trajectory for Identification 64

6.2 Real Sea Data for Verification 65

6.3 Second-Order Models $\quad 70$

6.4 Higher-Order Models $\quad 71$

$\begin{array}{ll}\text { 6.5 Model Performances } & 73\end{array}$

$\begin{array}{ll}\text { 6.6 Massless Models } & 75\end{array}$ 
$-6-$

6.7 Relationship of Link Model Parameters to Real Quantities

7. Conclusions and Recommendations 


\section{List of Figures}

Figure 1-1: The ARGO/JASON System 1.1

Figure 2-1: Free Body Diagram for Cable Element 2.2

Figure 2-2: Geometric Compatibility 2.3

Figure 2-3: Harmonic Cable Configuration Dynamics 2.4

Figure 2-4: Effective Tension 2.7 .1

Figure 2-5: Euler Angle Tranformation $\quad 2.7 .2$

Figure 3-1: Top View of AUTEC run $\quad 3.1$

Figure 3-2: Compressed AUTEC run 3.1

Figure 3-3: Simulated AUTEC Response Error 3.1

Figure 3-4: Top View of Typical Med Run $\quad 3.2$

Figure 3-5: Typical Time Series for Med Runs 3.2

Figure 3-6: Magnitude Response; Simulated vs. Real 3.2

Figure 3-7: Phase Lag Response; Simulated vs. Real 3.2

Figure 4-1: Simulated Step Response vs. Cable Length 4.1

Figure 4-2: Effect of Cable Length on Simulated Vehicle Response 4.1

Figure 4-3: Effect of Vehicle Weight on Simulated Step Response 4.1

Figure 4-4: Cable Evolution in Direct Point-to-Point Maneuver 4.2

Figure 4-5: Smart Point-to-Point Maneuver 4.2

Figure 5-1: Overall Identification and Verification Method 5.1

Figure 5-2: Learning-Model Method Flow Graph $\quad 5.2 .1$

Figure 5-3: Nelder-Meade Simplex Algorithm $\quad 5.2 .2$

Figure 6-1: Input/Output Spectrum for a 2500-meter Experiment 6.1

Figure 6-2: Identification Ship Velocity Series and Responses 6.1

Figure 6-3: 740-meter Experimental Data: Velocity 6.2

Figure 6-4: 740-meter Experimental Data: Position 6.2

Figure 6-5: 1200-meter Experimental Data: Velocity 6.2

Figure 6-6: 1200-meter Experimental Data: Position 6.2

Figure 6-7: 2500-meter Experimental Data: Velocity 6.2

Figure 6-8: 2500-meter Experimental Data: Position 6.2

$\begin{array}{ll}\text { Figure 6-9: Second-Order System } & 6.3\end{array}$

Figure 6-10: Higher-Ordered Cable Model $\quad 6.4$

Figure 6-11: First- vs. Second-Order Models: Identification Run 6.6

Figure 6-12: Fourth- vs. Eighth-Order Models: Identification Run 6.6

Figure 6-13: First- vs. Second-Order Models: Experimental Run 6.6

Figure 6-14: Fourth- vs. Eighth-Order Models: Experimental Run 6.6

Figure 6-15: Identification Run for 740 Meters: Velocity Error 6.8

Figure 6-16: Identification Run for 740 Meters: Position Error 6.8

Figure 6-17: Verification Run for 740 Meters: Velocity Error 6.8

Figure 6-18: Verification Run for 740 Meters: Position Error 6.8

Figure 6-19: Identification Run for 1200 Meters: Velocity Error 6.8

Figure 6-20: Identification Run for 1200 Meters: Position Error 6.8

Figure 6-21: Verification Run for 1200 Meters: Velocity Error 6.8

Figure 6-22: Verification Run for 1200 Meters: Position Error 6.8

Figure 6-23: Identification Run for 2500 Meters: Velocity Error 6.8

Figure 6-24: Identification Run for 2500 Meters: Position Error 6.8

Figure 6-25: Verification Run for 2500 Meters: Velocity Error 6.8

Figure 6-26: Verification Run for 2500 Meters: Position Error 6.8 
$-8-$

Figure 7-1: Actual and Simulated Fish Response--740 meters 7

Figure 7-2: Actual and Simulated Fish Response--1200 meters

Figure 7-3: Actual and Simulated Fish Response--2500 meters 
$-9-$

\section{List of Tables}

Table 3-I: Parameters for AUTEC Run

3.1

Table 3-II: "Tunable" Parameters for AUTEC Run

3.1

Table 3-III: Tyrrhenian Sea Parameters

3.2

Table 6-I: RMS Velocity Error, $\mathrm{m} / \mathrm{s}$

6.5

Table 6-II: RMS Position Error, $\mathrm{m} / \mathrm{s}$

6.5

Table 6-III: Comparison of Initial Parameter Estimates to Identified

6.7 Parameters 


\section{Chapter 1}

\section{Introduction}

\subsection{Motivation}

In ocean industry and science this decade, there has been increased interest in creating remote systems to work in deep water. The new remotely-operated vehicles (ROV's) are controlled from the surface or are entirely autonomous, and generally have capabilities similar to those of their manned counterparts, through remote cameras, manipulators, and other tools. The new technology can host a safer human working environment and indefinite mission duration; further, submersible design, no longer so strongly influenced by the need for life support, can take a more operation-oriented stance. As a result, the new vehicles can present a variety of completely new capabilities. Overall, there are clear advantages to using such systems, but the penalties have been found to be comparatively small.

The ARGO/JASON system developed at the Deep Submergence Laboratory of the Woods Hole Oceanographic Institution was conceived in this spirit to provide improved capabilities for the study of the seafloor. ARGO/JASON is a two-vehicle system operated entirely from the surface of the ocean, and is depicted in Figure 1-1. Major components include a very long cable (up to 6000 meters), the passive instrument platform ARGO, a slack 200-foot tether connection between the vehicles, and the maneuverable JASON vehicle, which is able to perform demanding manipulation and survey tasks on the seafloor. While ARGO carries only lights and sensor packages, JASON is equipped with thrusters and a manipulator, in addition to its own sensor arsenal. In the sequel, any ARGO-like vehicle is referred to as "the fish", or simply as "the vehicle". 


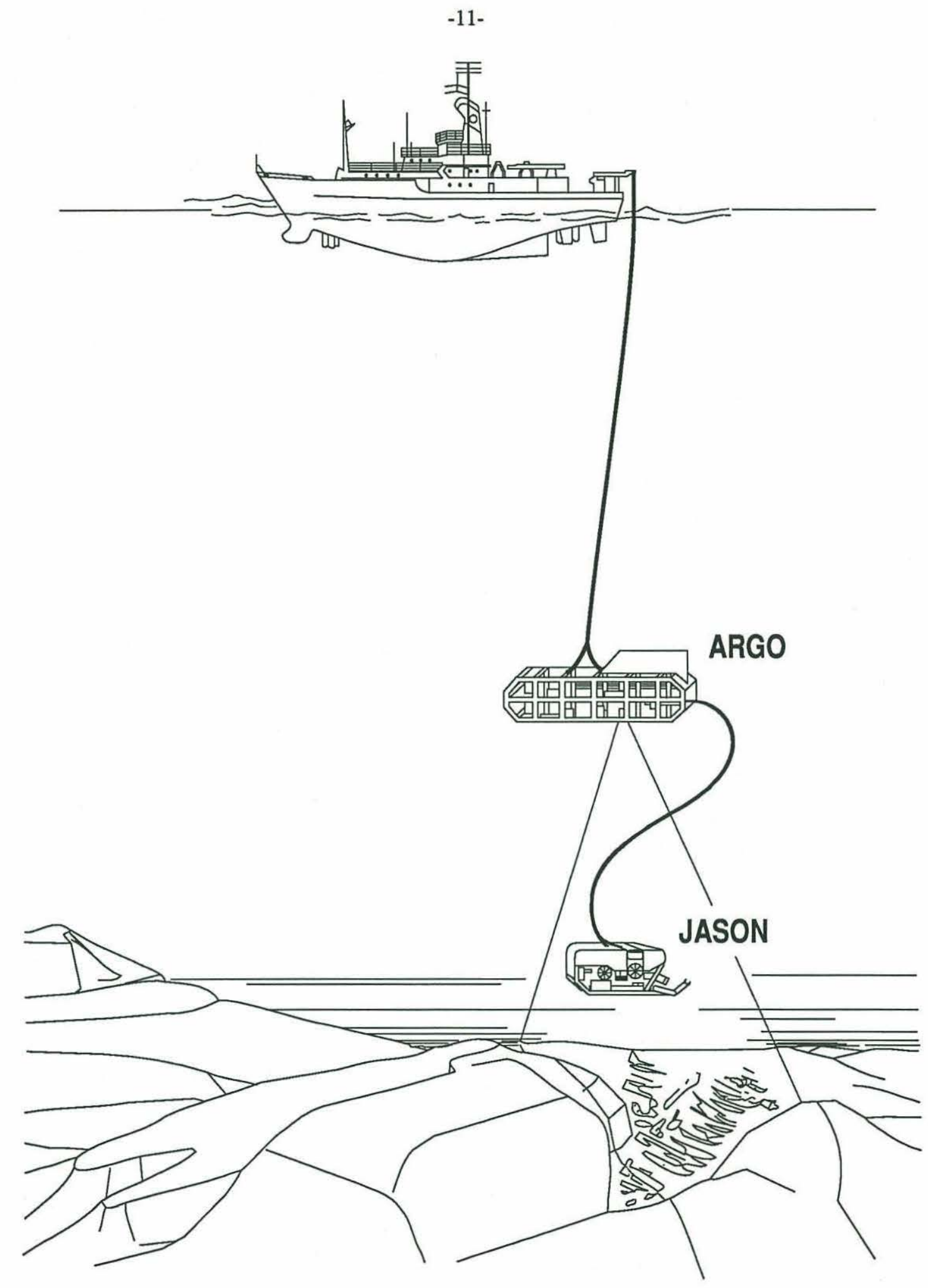

Figure 1-1: The ARGO/JASON System 
A first note about the configuration is that it decouples the high-frequency heave motions of the ship from JASON; this is essential for the precision maneuvering required by detailed surveys and manipulator use. While heave at the outboard sheave on the ship clearly has a strong interaction with ARGO, the length of tether between ARGO and JASON is meant to completely absorb these adverse vertical excursions. However, by design, we have accepted that the heave characteristics of ARGO may be similar to those of the ship in sea waves.

Another rationale for this configuration has to do with the horizontal location of ARGO/JASON: even in deep water the vehicles stay almost directly under the ship, due to ARGO's significant in-water weight. (High tension in the cable also keeps the system away from the ship's propulsion.) Thus, position control of JASON is comprised of two parts: local JASON commands to achieve desired motions in the world frame, and dynamic positioning of the surface ship to keep ARGO in the vicinity of JASON near the bottom. It is upon the second of these challenges that this work will focus.

High tension in the cable and its inclination at the lower end are the "forcing function" influencing the motions of ARGO. Clearly, they involve the ship motions (operator, dynamic positioning system, waves, and currents) and the overall state of the cable. For a long cable whose dynamics are described by partial differential equations with quadratic drag, the transfer function relating the top and bottom motions is not trivial. Intuition alone, however, can provide a basic description of this function: the cable acts like a low-pass filter, much like a damped pendulum.

In the ocean science community, there have been a number of cable simulation programs, mostly of the finite-difference variety. In this work, an approach is presented which takes advantage of the relatively smooth configuration of the cable by solving with a spectral method. All of these analytical forms are based on the fundamentals of mechanics and dynamics, and use the most basic physical quantities as parameters, such 
$-13-$

as cable mass per unit length, and Young's Modulus. In this sense, they are excellent models, and they possess an intrinsic consistency. Unfortunately, these models have two serious drawbacks in common: first, they are a computational burden, often requiring that realtime simulations be carried out on large, fast machines. More importantly, the analytical forms are not invertible; from a controls point of view, finite sets of differential equations are far preferred.

Conceivably, the system can be adequately approximated by finite-ordered differential equations, possibly with pure delays and probably nonlinear in nature. As one would expect for this type of approximation, however, the parameters for the short models may have dubious relations to real world quantities. This thesis presents a method for identification of these parametric models, using the universality of the analytical model to create them without using actual sea data. Verification, on the other hand, is achieved using real data from two cruises, with two different vehicles, at depths of 740,1200 , and 2500 meters. It is shown that this a priori identification/verification process can yield models which will work with existing control methodologies to provide improved system performance for a range of typical maneuvers.

\subsection{Outline of the Thesis}

Chapter 2 presents the derivation and implementation of the analytical model. Separate sections detail the fundamental dynamic concepts in the cable equations, and the conditioning of those results for use in a spectral approach. Vehicle dynamics are outlined, and notes for implementation are given concerning solving the static problem, and the dynamic response.

Qualitative verification of analytical model results against real sea data is shown in Chapter 3. First, a transient run is examined, in which there are significant sudden accelerations and long periods of nonzero towing speed. In addition, data taken for 
frequency analysis is presented. In both cases, the qualititative fits are found to be quite good, with a minimum of parameter tuning.

From the model derived in Chapter 2, Chapter 4 addresses several topics on the general behavior of the cable/vehicle system. In particular, typical pure time delays are quantified, and their origins are discussed; cable length and in-water vehicle weight are shown to be large factors in these delays and in the overall low-pass characteristics of the system. Further, the value of a dynamic model is illustrated by suggesting how ship motions might be optimized to enhance vehicle control during a point-to-point maneuver. Chapter 5 describes the identification and verification process to be used in the sequel, and discusses the overall approach. Actual model forms are derived in Chapter 6; emphasis is given to the influence of quadratic drag, and to higher-order forms which physically resemble the actual system. Results are presented for several of these models, and the potential of inertia-free models is addressed. In addition, the relationship between known physical quantities and parameters found via identification is briefly discussed. Finally, we point to some of the limitations and the practical aspects of the modelling process.

Conclusions and recommendations for future work are given in Chapter 7. 


\section{Chapter 2}

\section{Analytical Model}

\section{Derivation}

\subsection{Introduction}

The following cable and vehicle model has its origins in [Triantafyllou 86, Triantafyllou 87]. In it, advantage is taken of the smooth character of the cable shape, and the unwieldy finite-difference equations can be avoided in favor of a finite Fourier series with far fewer computational elements. The vehicle motions present a fairly standard problem in rigid-body dynamics, and they provide the boundary conditions for the lower end of the cable; the upper end motions are exactly those of the ship. One major feature of the model is that it solves the dynamics around a predefined static configuration, created by a current profile and/or steady tow speed condition.

\subsection{Equations of Motion and Statics}

Newton's Law applied to a differential element of the cable provides the equations of motion for the cable. We first note that if the unstretched length of an element is $d s$, then the stretched length $d p$ is related to $d s$ by the following equation:

$$
\frac{d p}{d s}=1+\varepsilon
$$

where $\varepsilon$ is the strain in the element. Letting $m$ be the mass per unit stretched length, $\mathbf{v}$ the absolute velocity vector of the element, $\mathbf{R}$ the applied force per unit stretched length, and F the internal force, Newton's Law gives (see Figure 2-1): 


$$
\frac{d}{d t}(m \mathbf{v} d p)=d \mathbf{F}+\mathbf{R} d p
$$

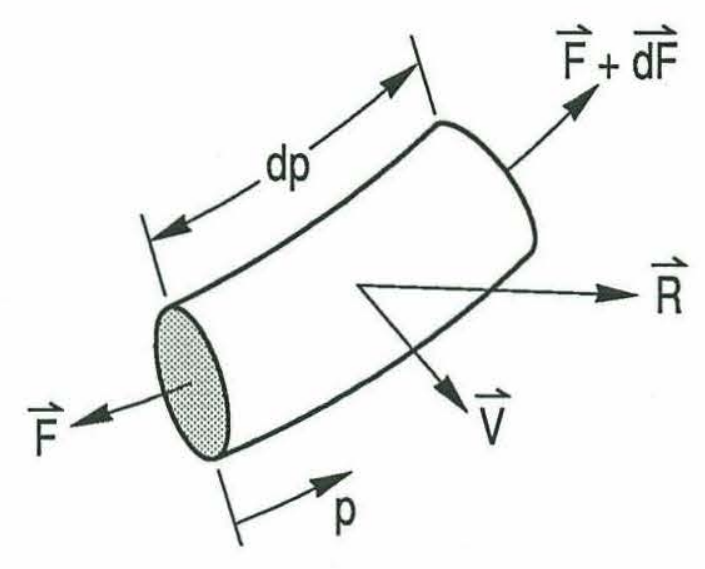

Figure 2-1: Free Body Diagram for Cable Element

Employing mass conservation, we are left with the simpler form of Newton's Law,

$$
m \frac{d \mathbf{v}}{d t}=\frac{d \mathbf{F}}{d p}+\mathbf{R}
$$

Several simplifications can be made at this stage. First, all bending moment effects are neglected; for a cable under high tension, the moments associated with the curvature alone are much higher than the internal moments due to bending stresses. The effects of torsional moments can be neglected also, since the eigenvalue problem worked out for torsional dynamics shows the first modes to be exceptionally fast. Similarly, the tension in the cable is assumed to be quasi-static; that is, there are no elastic travelling waves. Again, the eigenvalue problem gives very high frequency modes for this situation.

We choose to solve the problem in cable coordinates, and select the cable frame to coincide with the material tangential, normal, and binormal vectors. The rotation vector $\omega$ is used to account for time variance of the reference frame, and similarly the Darboux 
$-17-$

vector $\Omega$, which facilitates changes in the reference frame with respect to the Lagrangian cable coordinate $s$. For an arbitrary vector $\mathbf{f}$ in the cable frame, total derivatives can be expressed as:

$$
\begin{aligned}
& \frac{d \mathbf{f}}{d t}=\frac{\partial \mathbf{f}}{\partial t}+\omega \mathbf{x} \mathbf{f} \\
& \frac{d \mathbf{f}}{d s}=\frac{\partial \mathbf{f}}{\partial s}+\Omega \mathbf{x} \mathbf{f}
\end{aligned}
$$

Three Euler Angles are normally used to transform quantities between inertial and cable reference frames--it is convenient to express both the rotation and Darboux vectors in terms of these angles.

In general, $\mathbf{R}$ consists of gravity and drag effects, while $\mathbf{F}$ is simply ( $T \mathbf{t})$, where $\mathbf{t}$ is the tangent vector, and $T$ is the tension scalar. Assuming that a static configuration may contain some stretching, this result is given by

$$
0=\frac{d(T \mathbf{t})}{d t}+\mathbf{R}
$$

or

$$
0=\frac{\partial T}{\partial p} \mathbf{t}+T \frac{d \mathbf{t}}{d p}+\mathbf{R}
$$

Only the uncoupled two-dimensional problem will be dealt with in this work; in this special case, $\frac{d t}{d p}$ is the spatial rate of change of the cable's inclination angle $\phi$, and Frenet's relations from [Hildebrand 76] show that this derivative is in the binormal direction. We eventually arrive at the following two equations which completely describe the static configuration in the tangential and normal directions:

$$
0=w \sin (\phi)-\frac{d T}{d p}
$$


$-18$

$$
0=w \cos (\phi)-T \frac{d \phi}{d p}-\frac{1}{2} \rho C_{d} d U \sin (\phi)|U \sin (\phi)|
$$

where $\mathrm{w}$ is the cable weight in water per stretched unit length, and the velocity $U$ is provided by an arbitrary current field. Elongation of the cable is due to tension (Hooke's Law) and to hydrostatic pressure; both of these quantities typcially change with depth. See the description of the hydrostatic pressure effect in Appendix 2.7.1. Cable diameter $d$ is reduced from these elongations, through Poisson's Ratio. Finally, it is assumed that the cable is very nearly vertical, enough so that the tangential drag is negligible.

The initial conditions, $\phi$ and $T$ at the bottom of the cable, are found by setting the aggregate forces and moments on the vehicle equal to zero--this also provides the static inclination angle of the vehicle. In the sequel, the static cable angle is termed $\phi_{S}$. 


\subsection{Compatibility Relations}

The dynamics are described by (2.3) as well as the statics. The strategy will be to solve for the dynamics as deflections from the static configuration, which can be found beforehand. For this, we need to identify the geometric compatibility relations.

Compatibility can be expressed in terms of positions or velocities. The velocity-based results have the advantage that they are independent of the reference system, but in this work we have used the position-based relations. To begin, let the '0' subscript denote quantities in the static sense; deflections from these static configurations will be studied. In Figure 2-2, if $\mathbf{r}_{0}$ is the static position vector, and $\mathbf{r}$ is the dynamic position vector, then the deflection $\underline{\mathbf{r}}$ is defined as:

$$
\underline{\mathbf{r}}=\mathbf{r}-\mathbf{r}_{0}
$$

Letting $\Omega_{0}$ be the Darboux vector expressed in the static cable coordinate frame, we find that

$$
\frac{d \mathbf{r}}{d p_{0}}-\frac{d \mathbf{r}_{0}}{d p_{0}}=\frac{\partial\left(\mathbf{r}-\mathbf{r}_{0}\right)}{\partial p_{0}}+\Omega_{0} \mathbf{x}\left(\mathbf{r}-\mathbf{r}_{0}\right)
$$

Now, let

$$
\mathbf{t}=\frac{d \mathbf{r}}{d p}
$$

and

$$
\mathbf{t}_{0}=\frac{d \mathbf{r}_{0}}{d p_{0}}
$$

Combining terms into (2.11), we get 


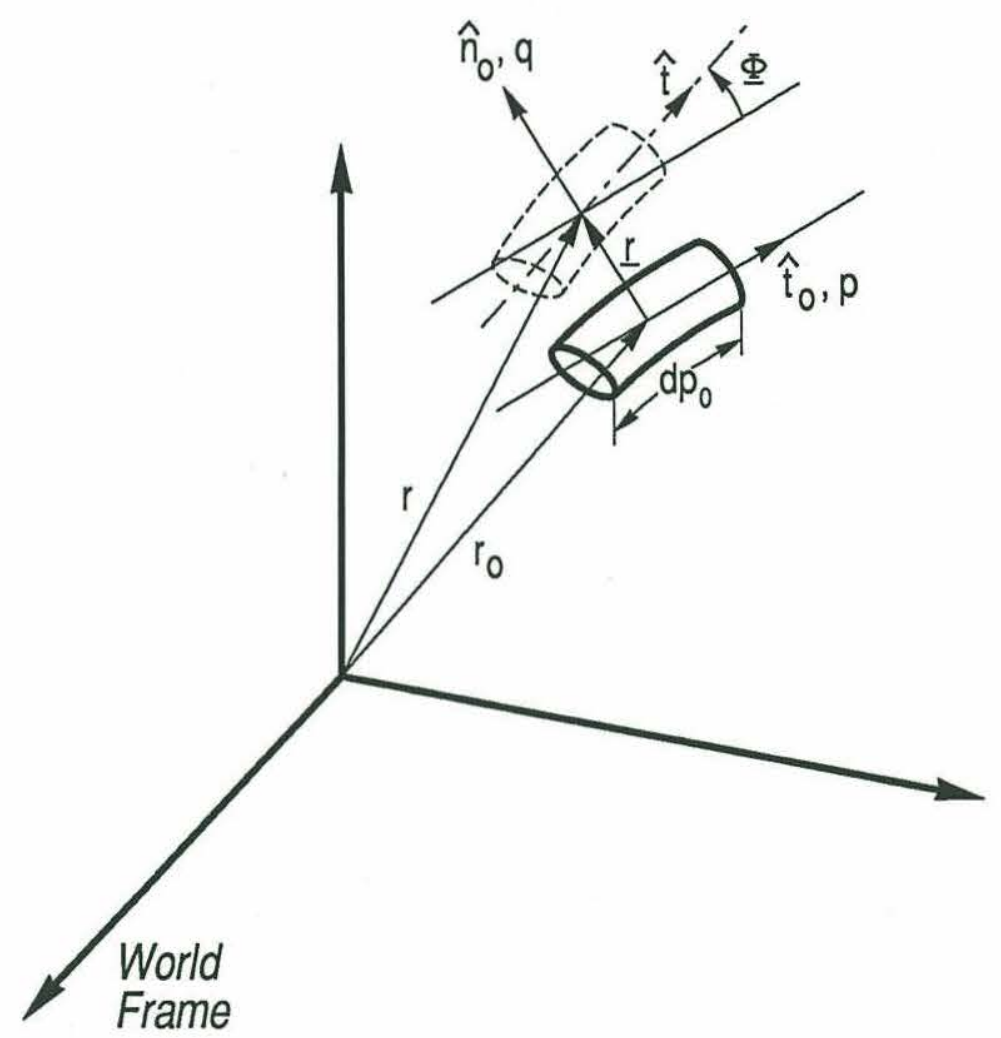

Figure 2-2: Geometric Compatibility

$$
(1+\varepsilon) \mathbf{t}-\left(1+\varepsilon_{0}\right) \mathbf{t}_{0}=\frac{\partial\left(\mathbf{r}-\mathbf{r}_{0}\right)}{\partial s}+\Omega_{0} \mathbf{x}\left(\mathbf{r}-\mathbf{r}_{0}\right)\left(1+\varepsilon_{0}\right.
$$

For reference, the velocity relations are easily found by differentiating this relation with respect to time. For the two-dimensional, position-based analysis, $\underline{\mathbf{r}}$ is projected onto the static cable reference system $\left\langle\mathbf{t}_{0}, \mathrm{n}_{0}\right\rangle$. Letting $p$ be the axial deflection and $q$ be the lateral deflection, $\underline{\mathbf{r}}$ is written as

$$
\underline{\mathbf{r}}=\mathbf{t}_{0} p+\mathbf{n}_{0} q
$$

and the dynamic tangent vector is expressed in terms of the angular deflection $\phi$, in the static cable reference frame: 


$$
\mathbf{t}=\mathbf{t}_{0} \cos (\phi)+\mathbf{n}_{0} \sin (\phi)
$$

For the two-dimensional case,

$$
\Omega_{0}=\frac{\partial \phi_{s}}{\partial p_{0}} \mathbf{b}_{0}
$$

Making a small-angle assumption for $\phi$, the final result turns out to be:

$$
\begin{aligned}
& \underline{\varepsilon}=\frac{\partial p}{\partial s}-q \frac{\partial \phi_{s}}{\partial s} \\
& \Phi(1+\varepsilon)=\frac{\partial q}{\partial s}+p \frac{\partial \phi_{s}}{\partial s}
\end{aligned}
$$

Each of these results is now incorporated in the equations of motion that were outlined above. First, (2.18) integrated over the length of the cable yields the dynamic elongation of the cable, taking into account the effects of static curvature. Namely,

$$
\Delta L=p_{L}-p_{0}-\int_{0}^{L} \frac{\partial \phi_{s}}{\partial s} q d s
$$

Along with the constitutive law, this gives the dynamic tension in the cable due to elongation and transverse deflections. It follows from the static equation (2.8), subject to our simplification of the axial dynamics, that

$$
T_{d}=\frac{E A}{L}\left[p_{L}-p_{0}-\int_{0}^{L} \frac{\partial \phi_{s}}{\partial s} q d s\right]-F_{d \mathbf{t}} p
$$

where $F_{d t}$ is the dynamic external force in the tangential direction, which acts beyond the force found for the static configuration.

Concerning the second compatibility relation (2.19), it is assumed that the dynamic angle 
due to axial motion of the cable is small with respect to the angle induced by dynamic lateral motions. This is motivated by and substantiated by consideration of the physical system: the vertical motions are small compared to the length. Coupling the dynamic and static terms in Newton's form, we have, after some work,

$$
m \frac{\partial^{2} q}{\partial^{2} t}=\left(T_{s}+T_{d}\right) \frac{\partial^{2} q}{\partial^{2} p}+T_{d} \frac{\partial \phi_{s}}{\partial p}+F_{d \mathbf{n}}
$$

Here, the pure static component from the coupling multiplication falls out, having been solved in the static case. Physically, the second term on the right side represents the cable's ability to straighten under a dynamic tension. Again, $F_{d \mathbf{n}}$ is the dynamic external force in the normal direction. 


\subsection{Solving the Modified Wave Equation}

The problem is greatly facilitated by some nondimensionalizations. First, let $\alpha=\frac{\partial \phi_{s}}{\partial s}$, and let $T_{r}$ be some representative tension in the cable(i.e., at the midpoint). The following definitions are made:

$$
\begin{aligned}
& \omega_{n}=\frac{\pi}{L} \sqrt{T_{r} / m} \\
& \tau=t \omega_{n} \\
& x=\frac{s}{L} \\
& \eta=\frac{q}{d} \\
& \xi=\frac{p}{d} \\
& \alpha_{0}=\frac{\alpha}{L} \\
& H_{s}=\frac{T_{s}}{T_{r}} \\
& H_{d}=\frac{T_{d}}{T_{r}}
\end{aligned}
$$

Noting a slight change in derivative notation, we begin with the dimensional form of the wave equation (2.22):

$$
m q_{t t}=\left(T_{d}+T_{s}\right) q_{s s}+T_{d} \alpha-F_{d \mathbf{n}}
$$

and using the new nondimensional variables, obtain 


$$
\eta_{\tau \tau}=\frac{T\left(H_{d}+H_{s}\right)}{m L^{2} \omega_{n}^{2}} \eta_{x x}+\frac{T H_{d} \alpha_{o} L}{m d \omega_{n}^{2}}-\frac{F_{d \mathbf{n}}}{m d \omega_{n}^{2}}
$$

$F_{d n}$ will be defined as the external normal force per unit length in the negative-q direction:

$$
F_{d \mathbf{n}}=\frac{1}{2} \rho C_{d} d\left|V_{r}\right| V_{r}
$$

where $V_{r}$ is composed of the combination of absolute incident flow, and the imposed flow due to the cable's own motion. About the equilibrium point, this normal force is

$$
F_{d \mathbf{n}}=\frac{1}{2} C_{d} d \rho_{w}\left(\left|q_{t}-\underline{U}\right|\left(q_{t}-\underline{U}\right)-|-\underline{U}|(-\underline{U})\right.
$$

with $\underline{U}=U \sin \left(\phi_{S}\right)$. Nondimensionalization and substitution into (2.25) gives:

$$
\eta_{\tau \tau}=\frac{T\left(H_{d}+H_{s}\right)}{m L^{2} \omega_{n}^{2}} \eta_{x x}+\frac{T H_{d} \alpha_{o}}{m d \omega_{n}^{2} L}-\frac{1}{2} D Z
$$

The simplified terms $D$ and $Z$ are given as follows:

$$
\begin{aligned}
& D=\frac{\rho d^{2}}{m} \\
& Z=C_{d}\left(\left|\eta_{\tau}-\frac{\underline{U}}{\omega_{n} d}\right|\left(\eta_{\tau}-\frac{\underline{U}}{\omega_{n} d}\right)+\frac{\underline{U}^{2}}{d^{2} \omega_{n}^{2}}\right)
\end{aligned}
$$

Making further use of the nondimensional terms, (2.28) becomes:

$$
\eta_{\tau \tau}=\frac{\left(H_{s}+H_{d}\right)}{\pi^{2}} \eta_{x x}+\frac{H_{d} \alpha_{o} L}{\pi^{2} d}-\frac{1}{2} D Z
$$

The solution to this partial differential equation is expanded using the Fourier theorem into an infinite sum of sinusoids plus additional terms to accomodate the boundary conditions, i. e., 


$$
\eta(x, t)=\eta_{1}(1-x)+\eta_{2} x+\sum_{n=1}^{\infty} Q_{n} \sin (n \pi x)
$$

The construction of a dynamic cable configuration is shown in Figure 2-3; a) shows the static catenary in a steady tow state, and b), c), and d) superimpose the first two, four, and eight harmonics, respectively. While complex cable shapes are possible with a small number of sinusoids, the true solution requires an infinite number.

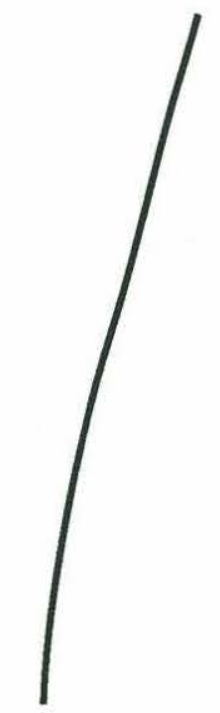

a) No

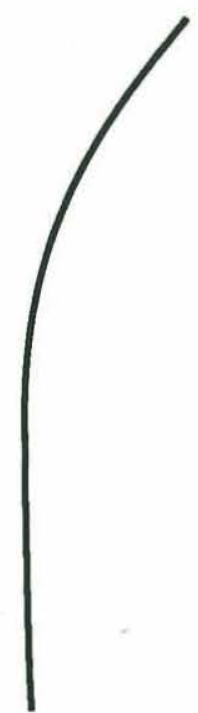

b) 2

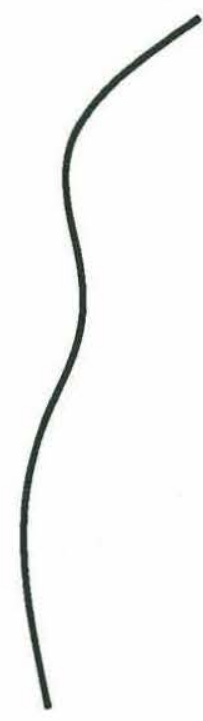

c) 4

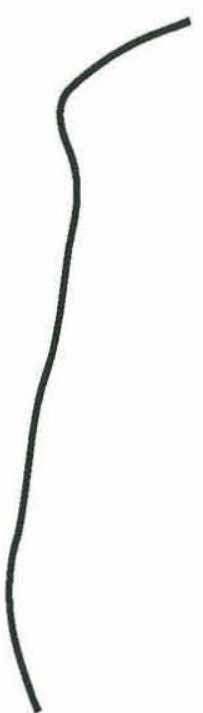

d) 8

Harmonics Harmonics Harmonics Harmonics

The nondimensional dynamic tension on the cable is expressed as (from (2.23)):

$$
H_{d}=\frac{E A d}{T_{r} L}\left(\xi_{2}-\xi_{2}-\int_{0}^{L} \alpha_{o} \eta d s\right)+\frac{F_{d \mathrm{t}} s}{T_{r}}
$$

where 


$$
F_{d \mathbf{t}}=\frac{1}{2} \rho C_{f}\left|V_{\mathrm{t}}\right| V_{\mathrm{t}} d
$$

In this case, it is assumed that $\cos \left(\phi_{S}\right)$ is small enough that tangential drag effects due to the flow field in the fixed frame can be neglected, when compared to those generated by the cable motion alone. Then $V_{\mathrm{t}}$ is the velocity of the cable itself, which can be averaged along the cable as

$$
V_{\mathbf{t}}=\frac{p_{2 t}-p_{1 t}}{2}
$$

or

$$
V_{\mathbf{t}}=\frac{1}{2} d \omega_{n}\left(\xi_{\tau 2}+\xi_{\tau 1}\right)
$$

Some manipulation gives a new form for (2.33):

$$
\begin{aligned}
H_{d}=\frac{E A d}{T_{r} L}\left(\xi_{2}-\xi_{1}-\int_{0}^{1} \alpha_{o} \eta d x\right)+ \\
\quad \frac{\rho C_{f} d^{3} \omega_{n}^{2} L x}{8 T_{r}}\left|\xi_{\tau 2}+\xi_{\tau 1}\right|\left(\xi_{\tau 2}+\xi_{\tau 1}\right)
\end{aligned}
$$

We will find more utility in a simpler form for the dimensionless dynamic tension: namely,

$$
H_{d}=H_{d}{ }^{o}+\delta H_{d} x \Xi
$$

where the definitions are made:

$$
H_{d}^{o}=\frac{E A d}{T_{r} L}\left(\xi_{2}-\xi_{1}-\int_{0}^{1} \alpha_{o} \eta d x\right)
$$




$$
\begin{aligned}
& \delta H_{d}=\frac{\rho C_{f} d^{3} \omega_{n}{ }^{2} L}{8 T_{r}} \\
& \Xi=\left|\xi_{\tau 2}+\xi_{\tau 1}\right|\left(\xi_{\tau 2}+\xi_{\tau 1}\right)
\end{aligned}
$$

Insertion of the solution from (2.32) into the expression for $H_{d}{ }^{o}$ yields

$$
\begin{gathered}
H_{d}{ }^{o}=\frac{E A d}{T_{r} L}\left(\xi_{2}-\xi_{1}-\int_{0}^{1} \alpha_{o}\left(\eta_{1}(1-x)+\eta_{2} x+\right.\right. \\
\left.\left.\sum_{n=1}^{n s} Q_{n} \sin (n \pi x)\right) d x\right)
\end{gathered}
$$

This is

$$
H_{d}{ }^{o}=\frac{E A d}{T_{r} L}\left(\xi_{2}-\xi_{1}-\eta_{1}\left(I_{2}-I_{1}\right)-\eta_{2} I_{1}-\sum_{n=1}^{n s} Q_{n} I_{3}{ }^{n}\right)
$$

where the integrals $I$ are defined as follows:

$$
\begin{aligned}
& I_{1}=\int_{0}^{1} \alpha_{o} x d x \\
& I_{2}=\int_{0}^{1} \alpha_{o} d x \\
& I_{3}^{n}=\int_{0}^{1} \alpha_{o} \sin (n \pi x) d x
\end{aligned}
$$

We let the static tension be comprised of a primary part and a spatially-varying part: $H_{s}=1+\delta H_{s}$. This leaves the wave equation (2.31) in the following form:

$$
\eta_{\tau \tau}=\frac{1}{\pi^{2}}\left(1+\delta H_{s}+H_{d}^{o}+\delta H_{d} x \Xi\right) \eta_{x x}+\frac{L}{\pi^{2} d} H_{d} \alpha_{o}-\frac{1}{2} D Z
$$


and insertion of the assumed solution (2.32) in its expanded form gives

$$
\begin{gathered}
\eta_{1 \tau \tau}(1-x)+\eta_{2 \tau \tau} x+\sum_{n=1}^{n s} Q_{n \tau} \sin (n \pi x)= \\
\frac{1+\delta H_{s}+H_{d}{ }^{o}+\delta H_{d} x \Xi}{\pi^{2}} \sum_{n=1}^{n s} Q_{n}(n \pi)^{2}(-\sin (n \pi x))+ \\
\frac{L}{\pi^{2} d}\left(H_{d}{ }^{o}+\delta H_{d} x \mathrm{~N}\right) \alpha_{o}-\frac{1}{2} D Z
\end{gathered}
$$

The equations are subsequently projected along the components of the Fourier expansions; i.e., each term is multiplied by $\sin (\mathrm{m} \pi \mathrm{x})$, for $\mathrm{m}$ an integer, and integrated over the length. Making use of the identities

$$
\begin{aligned}
& \int_{0}^{1} x \sin (n \pi x) d x=\frac{-(-1)^{n}}{n \pi} \\
& \int_{0}^{1} \sin (n \pi x) d x=\frac{1-(-1)^{n}}{n \pi}
\end{aligned}
$$

and of orthogonality, lengthy manipulation eventually gives

$$
\begin{aligned}
Q_{m \tau \tau}+m^{2} Q_{m}= & \frac{2}{m \pi}\left(-\eta_{1 \tau \tau}+\eta_{2 \tau \tau}(-1)^{m}\right)- \\
& 2 \sum_{n=1}^{n s} Q_{n} n^{2} \int_{0}^{1} \delta H_{s} \sin (n \pi x) \sin (m \pi x) d x- \\
& 2 \sum_{n=1}^{n s} Q_{n} n^{2} H_{d}^{o} \int_{0}^{1} \sin (n \pi x) \sin (m \pi x) d x- \\
& 2 \sum_{n=1}^{n s} Q_{n} n^{2} \delta H_{d} \mathrm{~N} \int_{0}^{1} \sin (n \pi x) \sin (m \pi x) x d x+
\end{aligned}
$$


$-29-$

$$
\begin{aligned}
& \frac{2 L H_{d}^{o}}{\pi^{2} d} \int_{0}^{1} \alpha_{o} \sin (m \pi x) d x+ \\
& \frac{2 L \delta H_{d} \mathrm{~N}}{\pi^{2} d} \int_{0}^{1} x \alpha_{o} \sin (m \pi x) d x- \\
& D \int_{0}^{1} Z \sin (m \pi x) d x
\end{aligned}
$$

For simplification, we define

$$
\begin{aligned}
& I_{4}^{n m}=-2 n^{2} \int_{0}^{1} \delta H_{s} \sin (n \pi x) \sin (m \pi x) d x \\
& I_{5}^{n m}=-2 n^{2} \delta H_{d} \int_{0}^{1} x \sin (n \pi x) \sin (m \pi x) d x \\
& I_{6}^{m}=\frac{2 L}{\pi^{2} d} \int_{0}^{1} \alpha_{0} \sin (m \pi x) d x \\
& I_{7}^{m}=\frac{2 L \delta H_{d}}{\pi^{2} d} \int_{0}^{1} x \alpha_{o} \sin (m \pi x) d x
\end{aligned}
$$

providing the equations in the form:

$$
\begin{aligned}
Q_{m \tau \tau}+m^{2} Q_{m}= & \frac{2}{m \pi}\left(-\eta_{1 \tau \tau}+\eta_{2 \tau \tau}(-1)^{m}\right)+\sum_{n=1}^{n s} Q_{n} I_{4}{ }^{n m}- \\
& Q_{m^{m}}{ }^{2} H_{d}^{o}+\Xi \sum_{n=1}^{n s} Q_{n} I_{5}^{n m}+I_{6}{ }^{m} H_{d}^{o}+\Xi I_{7}^{m}- \\
& D \int_{0}^{1} Z \sin (m \pi x) d x
\end{aligned}
$$

Also we note that 


$$
\begin{aligned}
& \int_{0}^{1} x \sin (n \pi x) \sin (m \pi x) d x=-2 n m \frac{1-(-1)^{n+m}}{\left(n^{2}-m^{2}\right)^{2}} \text { if } n \neq m \\
& \int_{0}^{1} x \sin (n \pi x) \sin (m \pi x) d x=\frac{1}{4} \text { if } n=m
\end{aligned}
$$

The final cable equations are contained in (2.49); each component presents a highly nonlinear problem. We note that for small deflections at least, the in-plane motions can be treated independently from the out-of-plane motions, but with exactly the same formulation. This will approximate the solution in three dimensions. When both motions are being modelled, care must be taken to see that the drag forces reflect the vector nature of the overall drag effect.

The vehicle dynamics must be next addressed to establish the response of the lower endpoint. 


\subsection{Vehicle Dynamics}

This section addresses the full three-dimensional dynamic vehicle response to uncoupled in-plane and out-of-plane cable motions. The Morison formulation is used to account for drag and inertial forces.

Newton's Law in translation and rotation gives:

$$
\begin{aligned}
& m \frac{d \mathbf{v}}{d t}=\mathbf{F}_{\text {added mass }}+\mathbf{F}_{\text {buoy }}+\mathbf{F}_{w t}+\mathbf{F}_{\text {tension }}+\mathbf{F}_{\text {drag }} \\
& \mathbf{I}_{\nu} \frac{d \omega}{d t}=\mathbf{M}_{\text {addedmass }}+\mathbf{M}_{\text {buoy }}+\mathbf{M}_{w t}+\mathbf{M}_{\text {tension }}+\mathbf{M}_{\text {drag }}
\end{aligned}
$$

It is simplest to let the velocity vector $\mathbf{v}$ and the rotation vector $\omega$ lie in the vehicle reference frame--for this reason, the Euler Angle rotations are again convenient. We define a matrix $\mathbf{C}_{v}$ which maps quantities in an inertial frame into the vehicle frame, through three Euler Angles. Further, $\mathbf{C}_{c}$ is a matrix which transforms quantities in the inertial frame to the cable reference frame, at the lower end of the cable.

The partial accelerations can be found by expanding the total derivatives with the rotation vector, as before. We find that:

$$
\begin{gathered}
\frac{\partial \mathbf{v}}{\partial t}=\left(m \mathbf{I}+\mathbf{A}_{m}\right)^{-1}\left(\mathbf{F}_{\text {buoy }}+\mathbf{F}_{w t}+\mathbf{F}_{\text {tension }}+\mathbf{F}_{\text {drag }}-m \omega \mathbf{x} \mathbf{v}\right) \\
\frac{\partial \omega}{\partial t}=\mathbf{I}_{v}^{-1}\left(\mathbf{M}_{\text {buoyancy }}+\mathbf{M}_{\text {weight }}+\mathbf{M}_{\text {tension }}+\mathbf{M}_{\text {drag }}-\right. \\
\left.\mathbf{r}_{a m} \mathbf{x} \mathbf{A}_{m} \frac{\partial \mathbf{v}}{\partial t}-\left(\mathbf{I}_{\nu} \omega\right) \mathbf{x} \omega\right)
\end{gathered}
$$

Here, $\mathbf{I}$ is the identity matrix, $\mathbf{A}_{m}$ is the added mass matrix, and $\mathbf{r}_{a m}$ is the vector which locates the added mass centers on the body.

The various forces are specified in the vehicle frame with the use of the transformation 
matrices $\mathbf{C}_{c}$ and $\mathbf{C}_{v}$ :

$$
\begin{aligned}
& \mathbf{F}_{\text {buoyancy }}=B \mathbf{C}_{\nu} \mathbf{z} \\
& \mathbf{F}_{\text {weight }}=-W \mathbf{C}_{\nu} \mathbf{z} \\
& \mathbf{F}_{\text {tension }}=T \mathbf{C}_{\nu} \mathbf{C}_{c}^{-1} \mathbf{t} \\
& \mathbf{F}_{\text {drag }}=-\frac{1}{2} \rho C_{d} \mathbf{v}(\mathbf{v} \cdot \mathbf{A})
\end{aligned}
$$

where $\mathbf{z}$ is the unit vector in the inertial vertical direction, and $\mathbf{A}$ is the area vector. The corresponding moments are the cross products of these forces with their respective location vectors.

With the above, we are able to propagate the body-referenced translational and rotational velocity; as for zeroeth derivatives, the location is preferred in inertial coordinates, and the three Euler Angles themselves. The world velocity is simply the inverse-transformed body-referenced velocity vector:

$$
\frac{\partial \mathbf{x}}{\partial t}=\mathbf{C}_{v}^{-1} \mathbf{v}
$$

The time rate of change of the Euler Angles is a little more difficult. First, the rotation vector in the world frame is the sum of the Euler Angle rates, each going through only some part of the whole transformation. Given the order of the rotations, it is possible through substitution to extract the individual Euler Angle rates, as a function of the current angles and the rotation vector; see Appendix 2.7.2. This transformation matrix we call S, and we get:

$$
\frac{\partial \Theta}{\partial t}=\mathbf{S} \omega
$$

where $\Omega$ is the vector of Euler Angles. It now remains only to transform the motions of 
$-33-$

the vehicle's cable connection point back into cable coordinates so that the cable problem can be propagated. The total motions can be considered as the summed effects of rotations of the vehicle and translation of its center, and we use the transformation matrices $\mathbf{C}_{c}$ and $\mathbf{C}_{v}$ as before to move between reference systems. The following results are in the cable frame at the bottom of the cable:

$$
\begin{aligned}
& \mathbf{x}_{c}=\mathbf{C}_{c}\left(\mathbf{C}_{v}^{-1} \mathbf{r}_{c}+\mathbf{x}\right) \\
& \frac{d \mathbf{x}_{c}}{d t}=\mathbf{C}_{c} \mathbf{C}_{v}^{-1}\left(\mathbf{v}+\omega \mathbf{x} \mathbf{r}_{c}\right) \\
& \frac{d^{2} \mathbf{x}_{c}}{d t^{2}}=\mathbf{C}_{c} \mathbf{C}_{v}^{-1}\left(\frac{\partial \mathbf{v}}{\partial t}+\frac{d \omega}{d t} \times \mathbf{r}_{c}+\omega \mathbf{x}\left(\omega \mathbf{x} \mathbf{r}_{c}\right)\right)
\end{aligned}
$$

Here, $\mathbf{r}_{c}$ is the cable connection point radius vector, in vehicle coordinates. These relations provide the lower-end quantities required for (2.49). For the top end of the cable, excitation motions need to be transformed into cable coordinates, similarly to what was done for the vehicle end. 


\subsection{Implementation Notes}

\subsubsection{Statics}

Before any of the dynamics can be addressed, it is necessary to determine the steady-state configuration of the system, in the presence of a flow field due to towing or a current profile. Equations (2.51) and (2.52), without added mass effects or derivative terms are the basis for finding the vehicle's orientation. The vehicle inclination and the cable angle at the point of attachment are the unknowns, and it is a simple matter to recursively solve for both quantities using (2.51) and (2.52). Following this, the cable angle and tension are integrated up to the surface using (2.8) and (2.9). Quantities which need to be saved during the space integration include the local tension, flow field, and curvature.

\subsubsection{Solving the Dynamics}

The resulting equations of Section $\mathbf{2 . 4}$ are actually solvable by any ordinary integrator. The two-dimensional problem was originally solved using Newmark's Method with a corrector loop, which is well suited to second-order nonlinear systems. However, if vehicle motions are to be followed in three dimensions, the differential equations in velocity are distinct from those in position, and we have chosen the standard Classical Runge-Kutta algorithm for propagation of all the first-order equations. Although the dynamics in surge for a long cable are known to be very slow, the dynamic tension itself may turn out to be very stiff: as a result, the integration time step must typically be much smaller than the horizontal response alone would dictate.

Obviously, for increasing resolution along the cable, and for increasing numbers of harmonics, the accuracy of the analytical model improves. In general, however, good results have been obtained with about thirty cable sections, and not many more than ten sinusoids. Certain responses, such as the step response, may require higher resolution in both respects, due to the importance of total cancellation at the lower end of the cable in 
the first seconds after the input is applied. It should be noted that an effect akin to aliasing can occur if the number of spectral components is too great compared to the number of cable segments; this will lead to incorrect, if not outright unstable, system responses.

\subsubsection{Forcing Functions}

With a relatively small arsenal of input capabilities, it is possible to investigate a wide array of cable behaviors. We have typically used the following:

1. Multiple superimposed, phase-shifted sinusoids in heave and surge

2. Arbitrary input from data file

3. Multiple ramped velocities

4. Multiple first-order velocities

5. Closed- or open-loop winch control

6. Superimposition of any of the above

One function not yet implemented but of great potential is a random sequence approximating the motions of a ship in a significant sea state. 


\subsection{Appendix}

\subsubsection{Effective Tension}

A cable section under high ambient pressure experiences an elongation due to the pressure, in addition to the stretching induced by pulling on the ends. The basis of this extra elongation is seen in Figure 2-4, where Archimedes' view of the cable element is appended with a tensile pressure effect to result in the actual physical state of the cable element. The contribution of this added pressure component is:

$$
T_{\text {eff }}=T_{\text {pulling }}+p A
$$

where $p$ is the ambient pressure, and $A$ is the cross-sectional area of the cable. In our work, the effective tension is most applicable when the static cable configuration is under study; the dynamic motions which affect this quantity are horizontal in general.

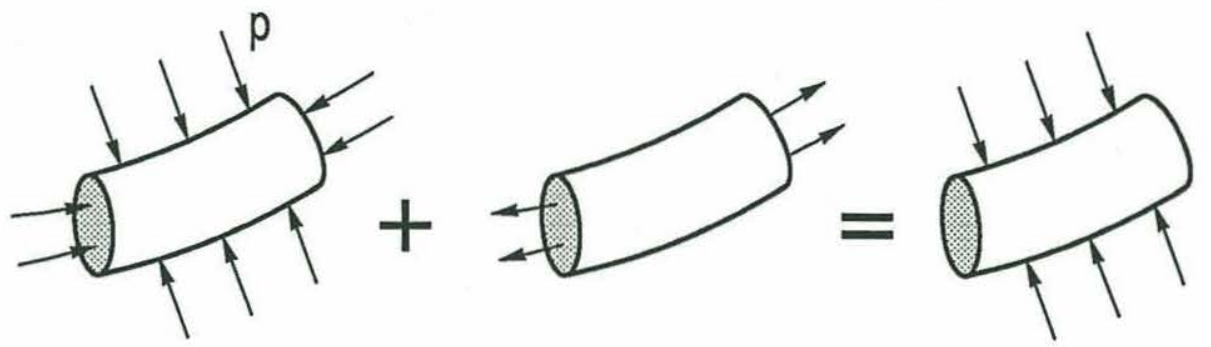

Figure 2-4: Effective Tension

\subsubsection{Propagating the Euler Angles}

This section describes how the matrix $\mathbf{S}$ can be found, given the order of rotation.

For this example, we let the rotations from the inertial frame occur in the following order: 
1. Rotation about the (inertial-frame) z-axis ( $\mathrm{z}$ ) by $\theta$

2. Rotation about the new y-axis $\left(\mathrm{y}_{1}\right)$ by $\phi$

3. Rotation about the final new $\mathrm{x}$-axis $\left(\mathrm{x}_{2}\right)$ by $\psi$

and the vehicle axes are $\left\langle x_{3}, y_{3}, z_{3}\right\rangle$. See Figure 2-5.

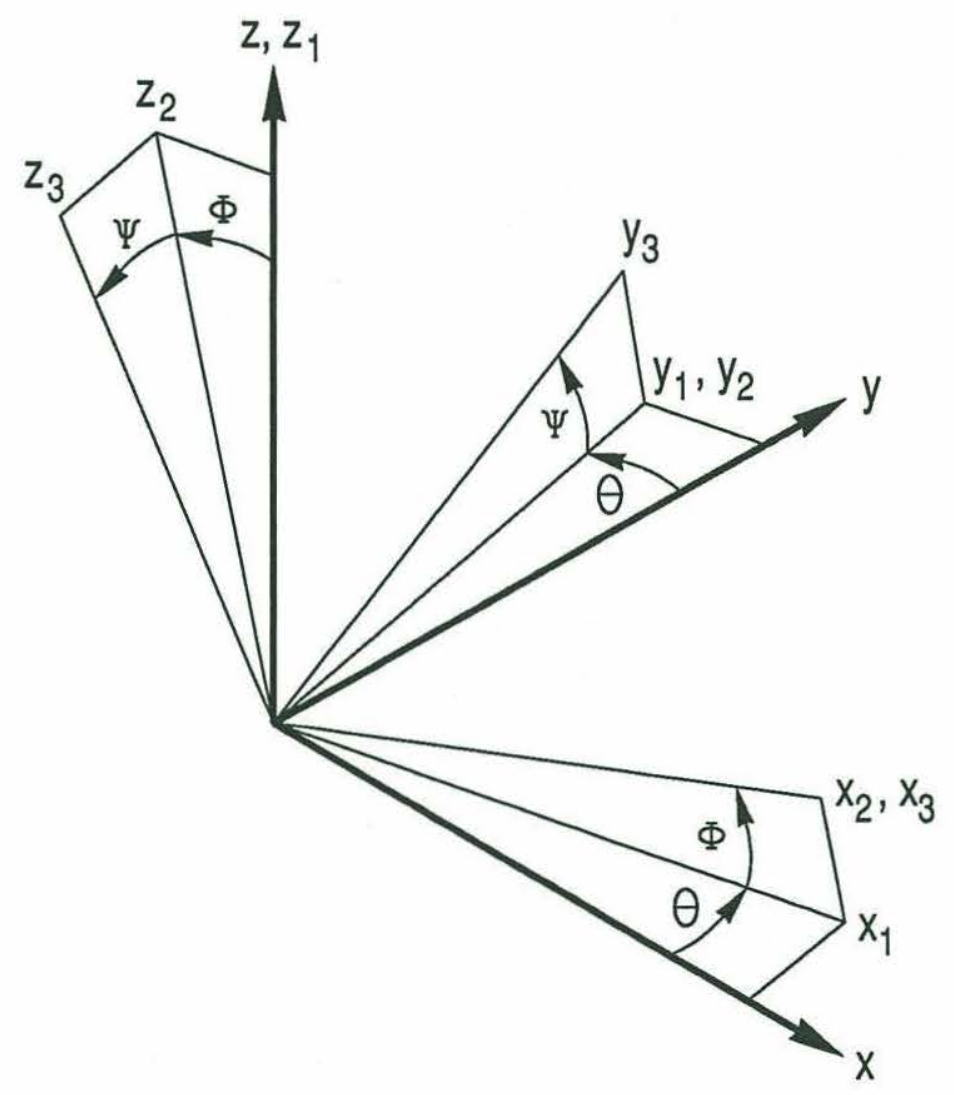

Figure 2-5: Euler Angle Tranformation

For each of the rotations, a simple transformation matrix can be found, and carrying them out successively leads to: $\mathbf{C}_{v}=\mathbf{C}_{\psi}, \mathbf{C}_{\phi} \mathbf{C}_{\theta}$. Each of the Euler rotations gives a distinct component of the rotation vector, and they are related to the intermediate unit vectors as follows: 


$$
\begin{aligned}
& \omega_{1}=\frac{\partial \psi}{\partial t} \mathbf{x}_{2} \\
& \omega_{2}=\mathbf{C}_{\psi} \frac{\partial \phi}{\partial t} \mathbf{y}_{1} \\
& \omega_{3}=\mathbf{C}_{\psi} \mathbf{C}_{\phi} \frac{\partial \theta}{\partial t} \mathbf{z}_{w}
\end{aligned}
$$

In fact, $\omega=\omega_{1}+\omega_{2}+\omega_{3}$, and this is sufficient knowledege to break up the rotation vector into its three components in the vehicle frame $<\omega_{x_{3}}, \omega_{y_{3}}, \omega_{z_{3}}>$. Next, substitution is used to isolate the Euler Angle time rates of change, in terms of the angles themselves and these rotation vector components. Working through it, we obtain a result for $\mathbf{S}$ :

$$
\mathbf{S}=\left[\begin{array}{lll}
0 & \frac{\sin (\psi)}{\cos (\phi)} & \frac{\cos (\psi)}{\cos (\phi)} \\
0 & \cos (\psi) & -\sin (\psi) \\
1 & \frac{\sin (\phi) \sin (\psi)}{\cos (\phi)} & \frac{\sin (\phi) \cos (\psi)}{\cos (\phi)}
\end{array}\right]
$$




\section{Chapter 3}

\section{Verification of the}

\section{Analytical Model}

The preceding chapter outlined a method for solving the dynamics of a towed cable and vehicle, about a static configuration created by an arbitrary current profile and/or due to a steady-state tow speed. In this section, the merits of this formulation are addressed qualitatively, by showing comparisons of model-predicted trajectories against real sea data. Two sets of real data from separate cruises are used, and comparisons are made in a time-domain as well as a frequency-domain sense.

\subsection{AUTEC Range Run}

The Navy's AUTEC Range in the Bahamas contains an extremely-well calibrated acoustic navigation net, and was the site of an April 1987 cruise to investigate the highfrequency transverse oscillations of a cable in tow. Although the primary measurements from this experiment were via accelerometers placed in bottles along the cable length, acoustic pingers on the ship, along the cable, and on the steel sphere at the bottom gave good overall tracking data. Table 3-I shows the relevant physical parameters for the system.

Although the ship tracks were generally not on a straight line, there were data sets appropriate for the purpose of verifying the analytical model. The assumption that outof-plane motions are decoupled from the in-plane ones, if deflections are small, is the key to compressing two-dimensional ship and vehicle trajectories into one dimension. Figure 3-1 shows a birds-eye view of the data set of interest. By regarding only the projections along a line about forty-five degrees from the eastern direction, it is possible to derive 


\begin{tabular}{|l|c|}
\hline Cable Density, $\frac{\mathrm{kg}}{\mathrm{m}^{3}}$ & 4400 \\
\hline Cable Diameter, $\mathrm{m}$ & .0173 \\
\hline Cable Length, $\mathrm{m}$ & 1200 \\
\hline Young's Modulus, $\frac{\mathrm{N}}{\mathrm{m}^{2}}$ & $6.2 \times 10^{10}$ \\
\hline Vehicle Wt. in Water, $\mathrm{N}$ & 19,170 \\
\hline Vehicle Mass, $\mathrm{kg}$ & 2240 \\
\hline Vehicle Projected Area, $\mathrm{m}^{2}$ & .56 \\
\hline Water Density, $\frac{\mathrm{kg}}{\mathrm{m}^{3}}$ & 1025 \\
\hline
\end{tabular}

Table 3-I: Parameters for AUTEC Run

quite a good approximation to two-dimensional motion. The result of this compression is illustrated in Figure 3-2, and these points now comprise the norm to be emulated by simulation.

It is clear from the Figures that a steady-state offset of around 40 meters separates the ship and fish positions along the compression line: it is due to possible current effects, and the separation between the outboard sheave and the ship's transducer. Neither of these quantities was available at the time of this writing, and a simplification has been adopted in which the dynamic effects of small offsets in position are ignored, and the model predicts responses as if there were none. The simplest way to avoid relying on a 
$-41-$

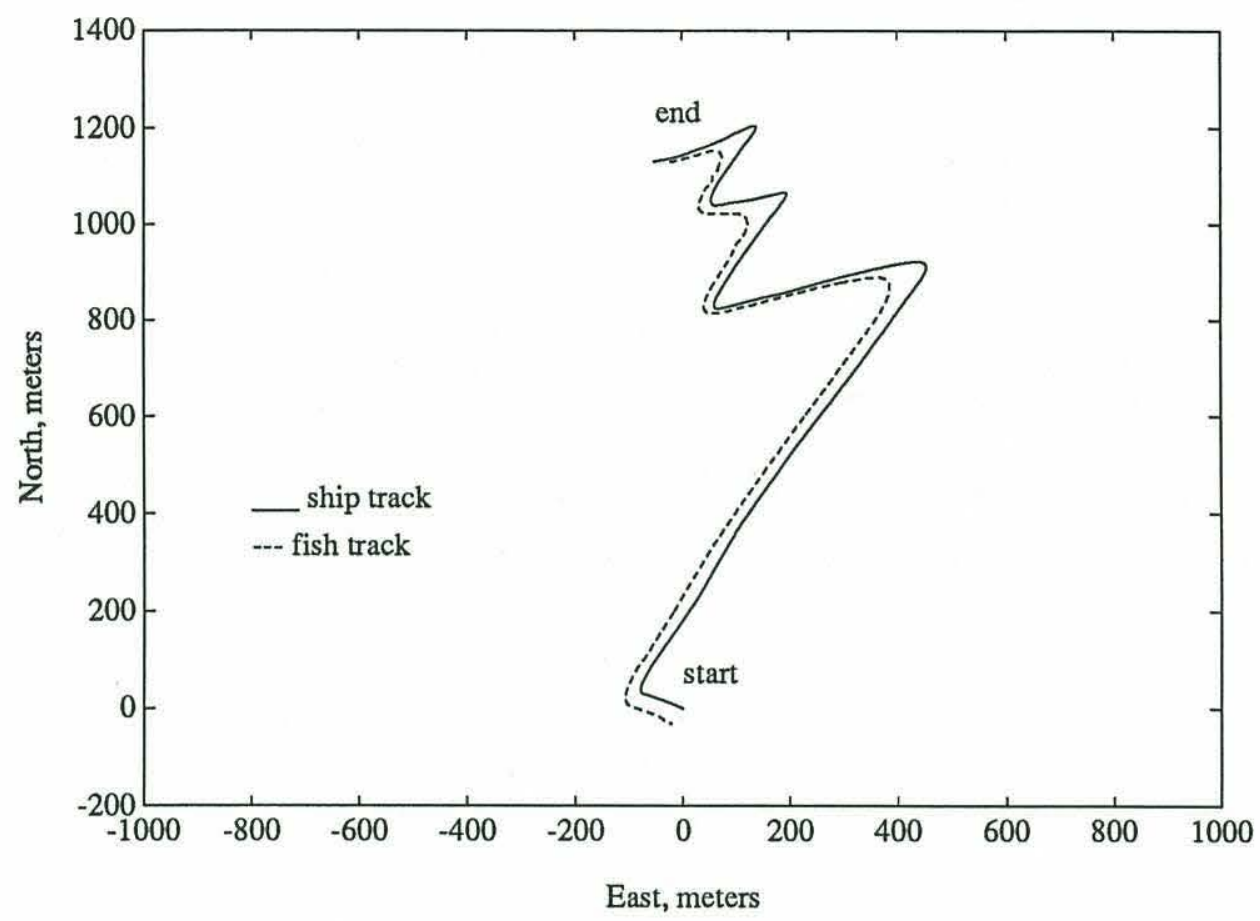

Figure 3-1: Top View of AUTEC run

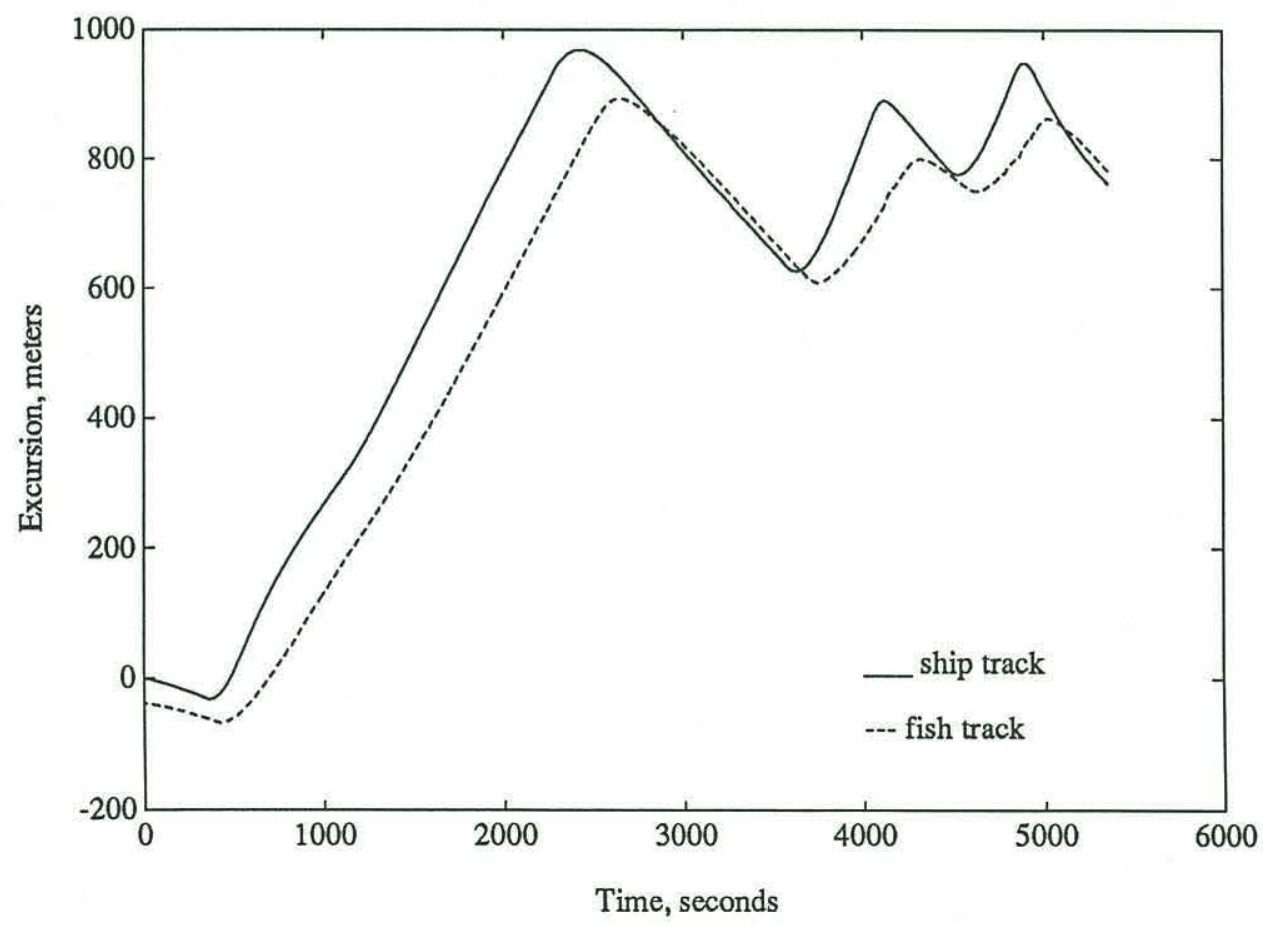

Figure 3-2: Compressed AUTEC run 
constant bias in correlation is to stress the velocity fits instead. This is the view adhered to in the sequel, because all dynamic effects due to the offsets have been found to be small. Position errors obtained by direct subtraction of the offset are often referenced as a matter of interest, but are considered less meaningful, and the identification schemes do not use them.

It is also apparent that the cable is probably not in a steady state at the start of the file and as a result, the initial configuration of the system cannot be exactly (or otherwise) defined. In other words, the known velocity profile at [time $=0]$, plus the ship offset, does not necessarily imply in simulation the offset given by the data. For this reason, our cable will always be assumed at zero deflection and speed at the start; since this assumption puts additional offset into the cable, our earlier statements about the importance of the velocity correlations, and a more casual treatment of the position bias, fit in well with this plan.

Figure 3-3 shows the performance of the analytical model on this data set. The rootmean-squared velocity error is $.028 \mathrm{~m} / \mathrm{s}$ (rms velocity is $.351 \mathrm{~m} / \mathrm{s}$ ), and the rms position error is $5.0 \mathrm{~m}$; the maximum position error is $10.4 \mathrm{~m}$, in excursions of nearly one thousand. The fundamental note for these plots is that only one parameter was "tuned" to enhance the fit; this was the drag coefficient of the cable, set tentatively at 1.6. A good number of other quantities were known exactly, such as the cable stiffness, water density, vehicle weight in water, et cetera. Others, such as the drag characteristics of the vehicle, and the added masses of the vehicle and cable, were estimated, and the output seemed less sensitive to changes in these values than in the cable $\mathrm{C}_{d}$. Table 3-II shows the "tunable" values used for the final run. 

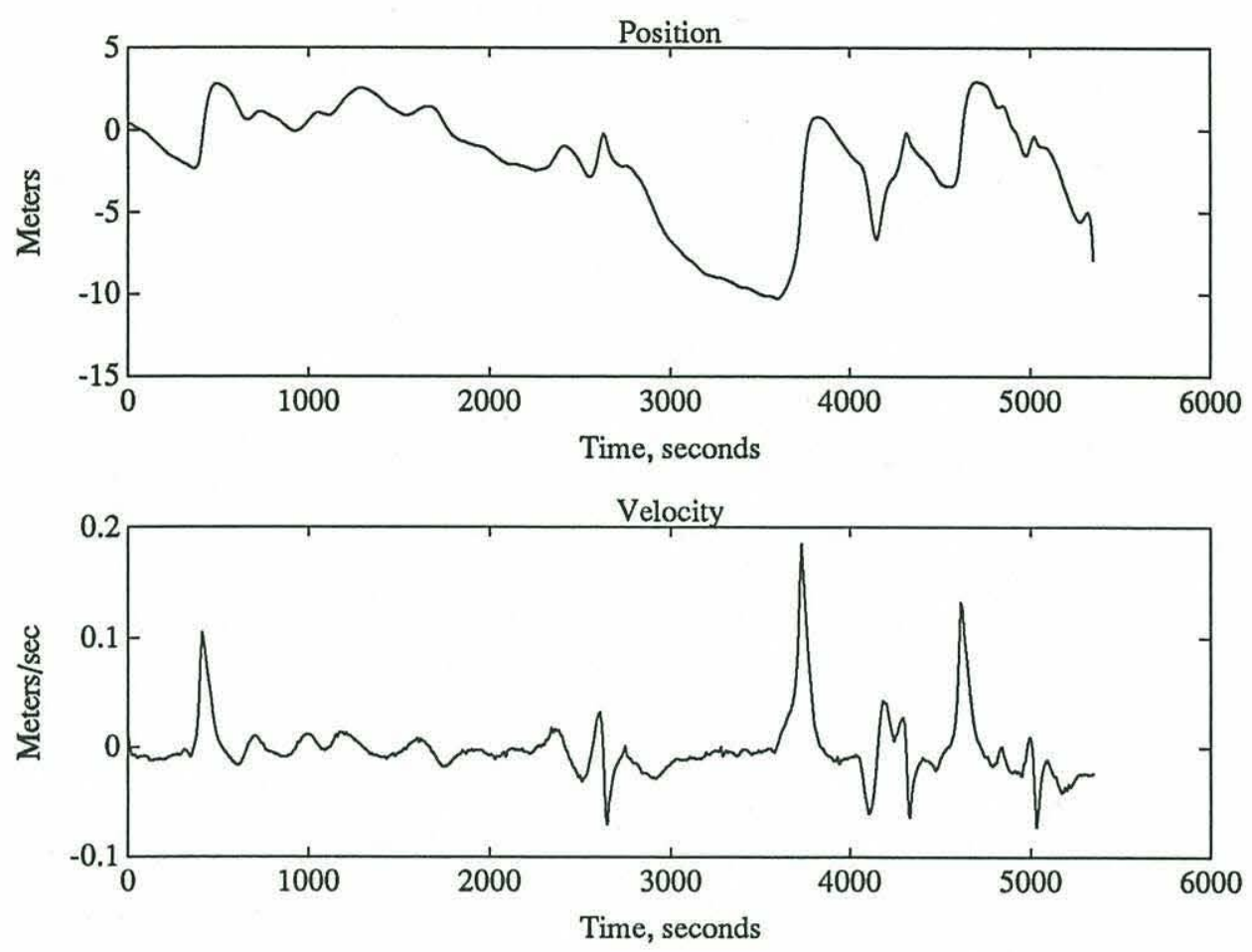

Figure 3-3: Simulated AUTEC Response Error

\begin{tabular}{|l|c|}
\hline Cable $_{f}$ & .08 \\
\hline Cable $C_{D}$ & 1.6 \\
\hline Cable Added Mass, $\frac{\mathrm{kg}}{\mathrm{m}}$ & .24 \\
\hline Vehicle $\mathrm{C}_{D}$ & .4 \\
\hline Vehicle Added Mass, $\mathrm{kg}$ & 45 \\
\hline
\end{tabular}

Table 3-II: "Tunable" Parameters for AUTEC Run 


\subsection{Tyrrhenian Sea Data}

The second data set of interest was obtained during August 1988 in the Tyrrhenian Sea. The physical parameters of note are given in Table 3-III. The important changes from the AUTEC parameters were the vehicle, now a camera sled named MANGUS, and the cable length of 2500 meters. Most notably, the ship motions here were chosen for frequency analysis. The use of a dynamic positioning system made it possible to effect rough sinusoidal trajectories in one dimension, by closing both the heading and east-west regulator loops, and then using a joystick to simply move the ship fore and aft along a longitudinal line. An actual top view of one of these maneuvers, with fixes available every ten seconds, is depicted in Figure 3-4. The fish position is offset due to a small current field predominantly southeast to northwest, and the inboard location of the ship transducer. It is clear from the time-series plot in Figure 3-5 that our intution about the cable acting as a filter is correct: the measurable amplitude attenuation and phase lag substantiate this.

A number of runs were made at different amplitudes and frequencies, and the best of them, in Bode Plot form in Figures 3-6 and 3-7, are overlaid on curves generated by the analytical model for the scenarios of the data. Clearly, the amplitude-dependence of the frequency response is suggestive of the quadratic nonlinearity to be expected in a system with fluid drag. Assuming that smooth curves drawn through the actual data points represent the physical system, in amplitude we see that the simulation error is limited to about a decibel for the low-frequency fifty-meter runs. At twenty-five meters amplitude, the error is much lower. Phase lag errors are small in fifty-meter case (under five degrees), but are larger for the twenty-five-meter case, approaching twenty degrees. These results confirm the overall viability of the analytical model for predicting real sea data in the frequency domain, especially since the parameters for the simulation are taken directly from those used in the AUTEC run--there was no tuning. 


\begin{tabular}{|l|c|}
\hline Cable Density, $\frac{\mathrm{kg}}{\mathrm{m}^{3}}$ & 4400 \\
\hline Cable Diameter, $\mathrm{m}$ & .0173 \\
\hline Cable Length, $\mathrm{m}$ & 2500 \\
\hline Young's Modulus, $\frac{\mathrm{N}}{\mathrm{m}^{2}}$ & $6.2 \times 10^{10}$ \\
\hline Vehicle Wt. in Water, $\mathrm{N}$ & 6000 \\
\hline Vehicle Mass, $\mathrm{kg}$ & 760 \\
\hline Vehicle Projected Area, $\mathrm{m}^{2}$ & .52 \\
\hline Water Density, $\frac{\mathrm{kg}}{\mathrm{m}^{3}}$ & 1025 \\
\hline Cable $\mathrm{C}_{\mathrm{f}}$ & .08 \\
\hline Cable $\mathrm{C}_{\mathrm{D}}$ & 1.6 \\
\hline Cable Added Mass, $\frac{\mathrm{kg}}{\mathrm{m}}$ & .24 \\
\hline Vehicle $\mathrm{C}_{\mathrm{D}}$ & 1.6 \\
\hline Vehicle Added Mass, $\mathrm{kg}$ & 545 \\
\hline
\end{tabular}

Table 3-III: Tyrrhenian Sea Parameters 


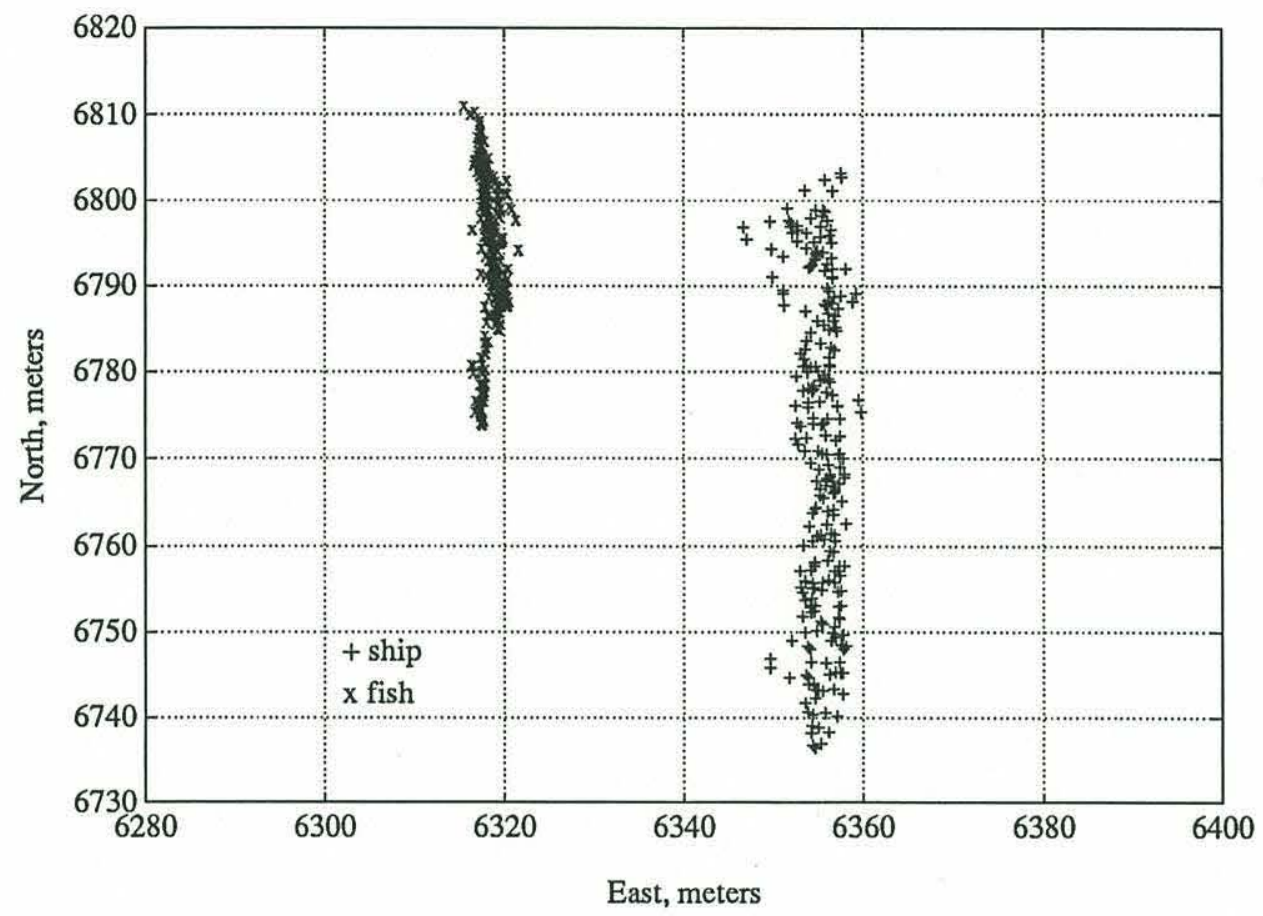

Figure 3-4: Top View of Typical Med Run

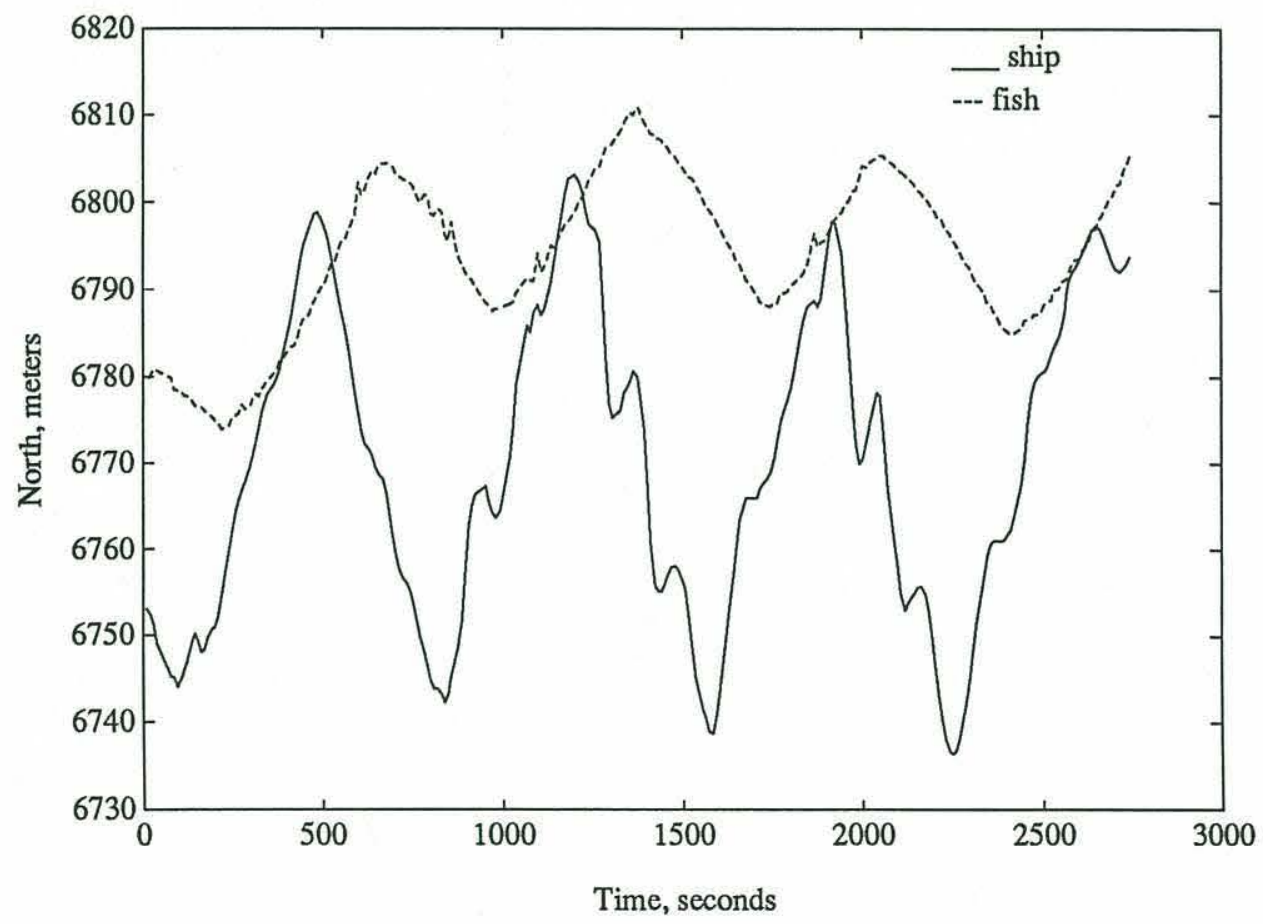

Figure 3-5: Typical Time Series for Med Runs 


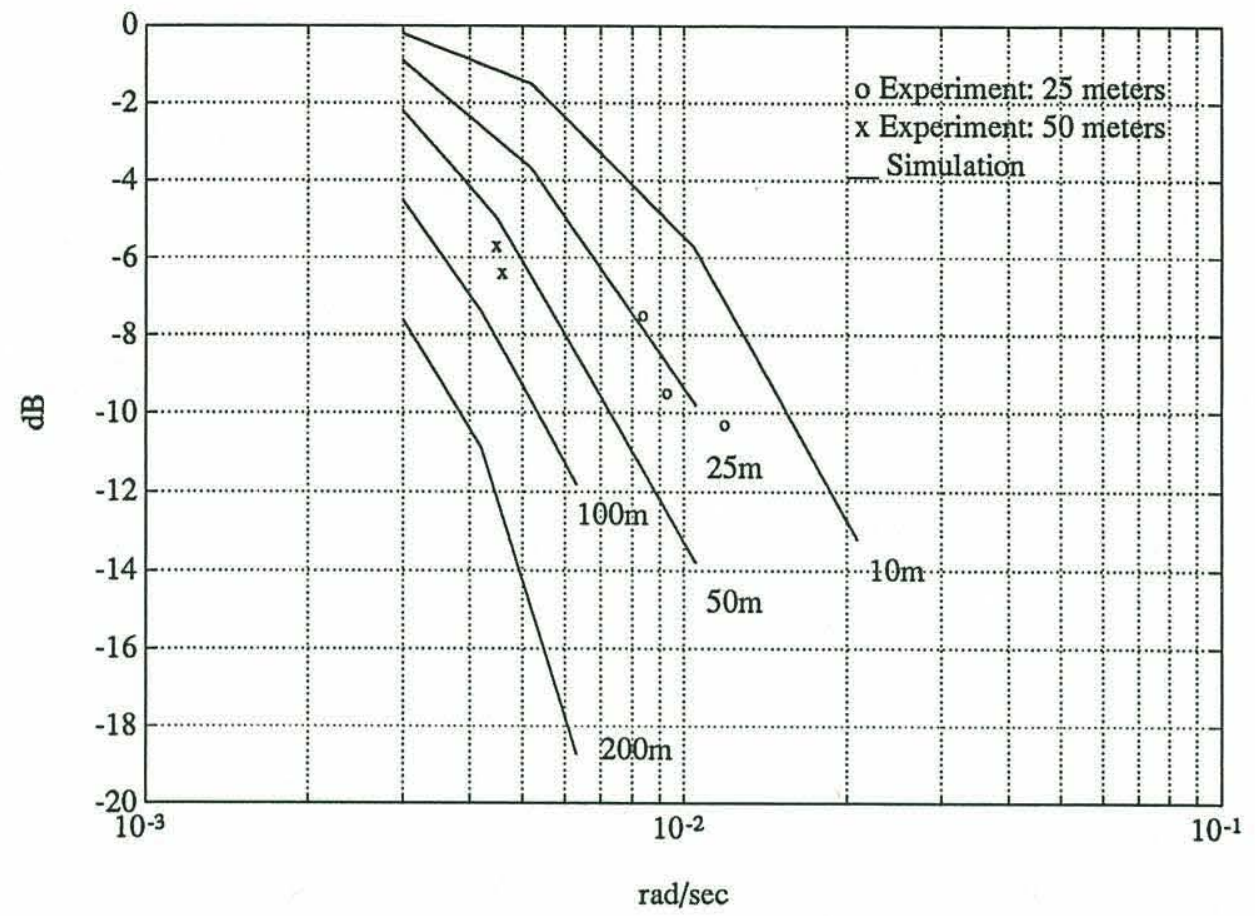

Figure 3-6: Magnitude Response; Simulated vs. Real

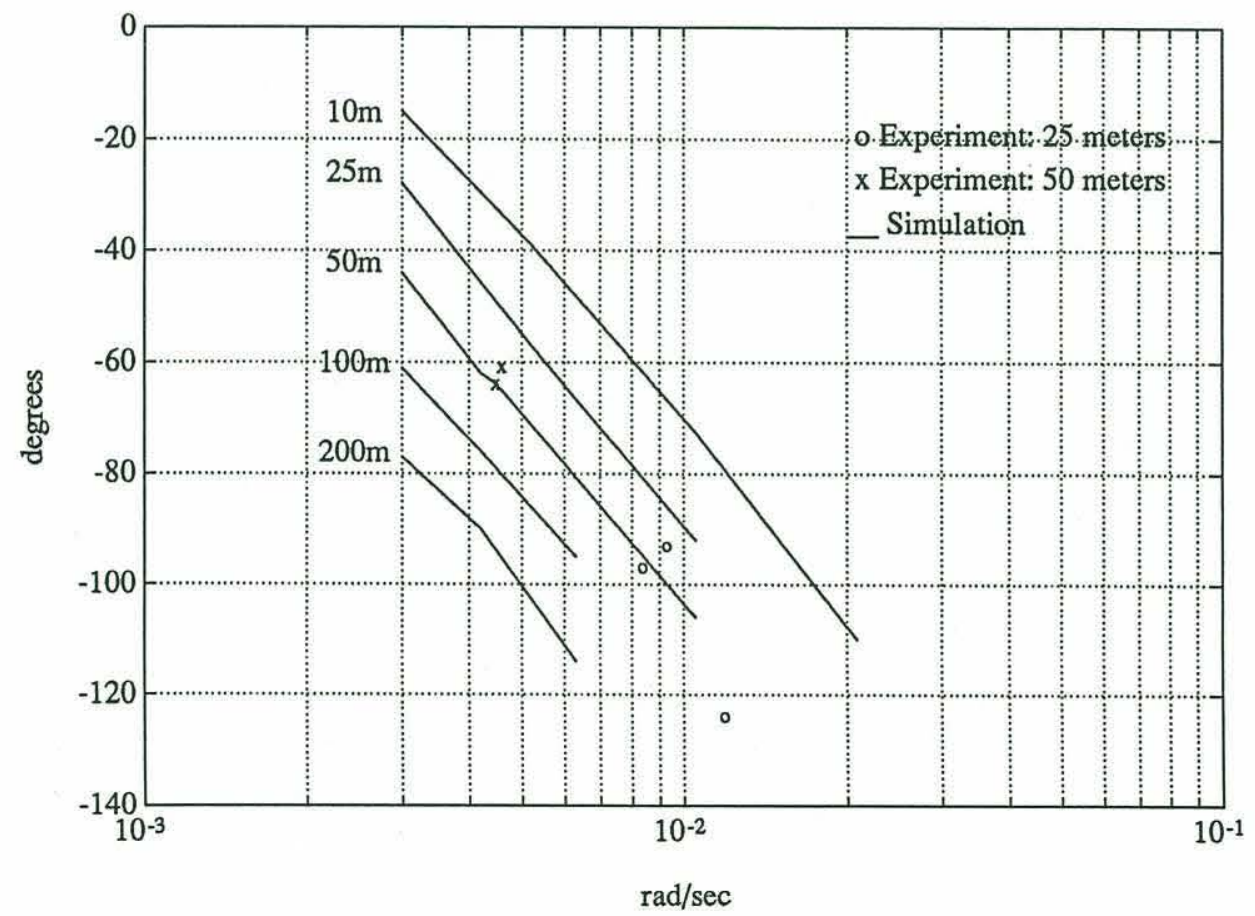

Figure 3-7: Phase Lag Response; Simulated vs. Real 


\subsection{Conclusions}

It has been shown that the analytical model can, without too much uncertainty, provide a good estimate of the system response to a one-dimensional ship trajectory. Two regimes were qualitatively considered: one in which significant deflections arise due to nonzero towing speeds, and another comprising smaller zero-mean motions which test the model's ability to simulate travelling waves. As stated previously, the real power of this model is evident when considering that all of its parameters are fundamental quantities, and that the only "tuning" was in adjustment of the cable $C_{d}$. This universality promises good simulation results for artificial systems, with a minimum of guesswork; in later chapters, it will be the backbone of the identification process.

Looking beyond this thesis, the capability for surge response prediction is not the only use for the model. In heave, the simplest model of a spring with a lumped mass and drag source is good only in a very few cases. More likely, the coupled pitch and heave dynamics of the vehicle are important, and the tangential drag of the cable may be significant. Most importantly, significant lateral deflections of the cable tend to couple the vertical and horizontal dynamics along its length; this is a particularly complex and important characteristic. With the model's ability to capture these effects, we have used

it, and are still using it, to address the issues of maximum cable tension and possible snap loads during operation. Future cruises should provide some data to verify this aspect of the model, and further facilitate understanding of these dynamics. 


\section{Chapter 4}

\section{Observations on Cable Behavior}

The Bode Plot of Chapter 3 showed the fundamental low-pass filtering characteristic of the cable dynamics, as did the AUTEC comparison presented there also. Knowing that the system is in fact governed by partial differential equations, the real dynamics are expected to be somewhat more complex than the Bode Plot alone would suggest; a linear model taken directly from that result would be valid only for a specific amplitude, and its performance might decay at frequencies far from the nominal one(s) chosen for identification. Although the inclusion of quadratic drag in nonlinear models can close the gap somewhat, it will be useful to clarify the real dynamics of the system. The purpose of this chapter is to elucidate a little more specifically these real dynamics.

\subsection{Time Delay}

A good first approximation to the cable problem is that of a taut string in a viscous medium. These dynamics are given by:

$$
m \frac{\partial^{2} q}{\partial t^{2}}=T \frac{\partial^{2} q}{\partial s^{2}}+\mathbf{F}_{d \mathbf{n}}
$$

as before where $m$ is the cable mass per unit length, $q$ is the lateral deflection, $T$ is the cable tension, $s$ is the Lagrangian cable coordinate, and $\mathbf{F}_{d \mathbf{n}}$ is the normal drag force per unit length. If the cable weight in water is zero, and the initial configuration of the cable is completely vertical and straight, the analytical model addresses exactly this form. A depth-dependent tension is easily added to the formulation.

The familiar travelling wave provides a solution to this problem, provided that the 
damping is "small". However, for the problem at hand, viscous forces are very strong, and what was a discrete wave packet in the inviscid case is now smeared over a much larger vertical length, and may travel quite slowly. In either case, the separation in a dynamic sense between the top and the bottom is real, and a pure time delay is inescapable. Figure 4-1 shows the velocity response of a MANGUS-like vehicle on various cable lengths to a (very nearly) step input in velocity of .5 meters/second.

The obvious trend is that longer cable lengths are subject to longer pure time delays; for the lengths in the figure, this delay may vary from around eight seconds to nearly thirty. In addition, once the vehicle has started to move, it is clear that the shorter cable approaches the steady state speed of .5 meters/second significantly faster than the longer lengths. We may be able to explain the first characteristic by noting the similarity in wave speed for the three configurations, which is $\sqrt{T / m}$. The pure delays based only on this wave speed are estimated roughly as 5.7, 10.2, and 20.2 seconds for the 500-, 1000-, and 2500-meter lengths, respectively. Differences in velocity after the pure delay are primarily due to the catenary "strength" underneath the ship; greater cable angles for shorter cables result in larger restoring forces. The nonminimum-phase behavior indicated by the 2500-meter response is an artificial byproduct of the spectral approximation to a partial differential equation, and is ignorable, insofar as runs made with a great many more spectral components will erase it, but give similar responses otherwise.

Extending these principles to a case of random excitation illustrates further the filtering behavior of the cable, and the effects of its length. In Figure 4-2 is superimposed the responses of the MANGUS vehicle on varying cable lengths, to a filtered and intentionally-biased random sequence (It is the same sequence used for identification in a later chapter). The conclusion is identical: a short cable posesses faster filter dynamics than a long one. 


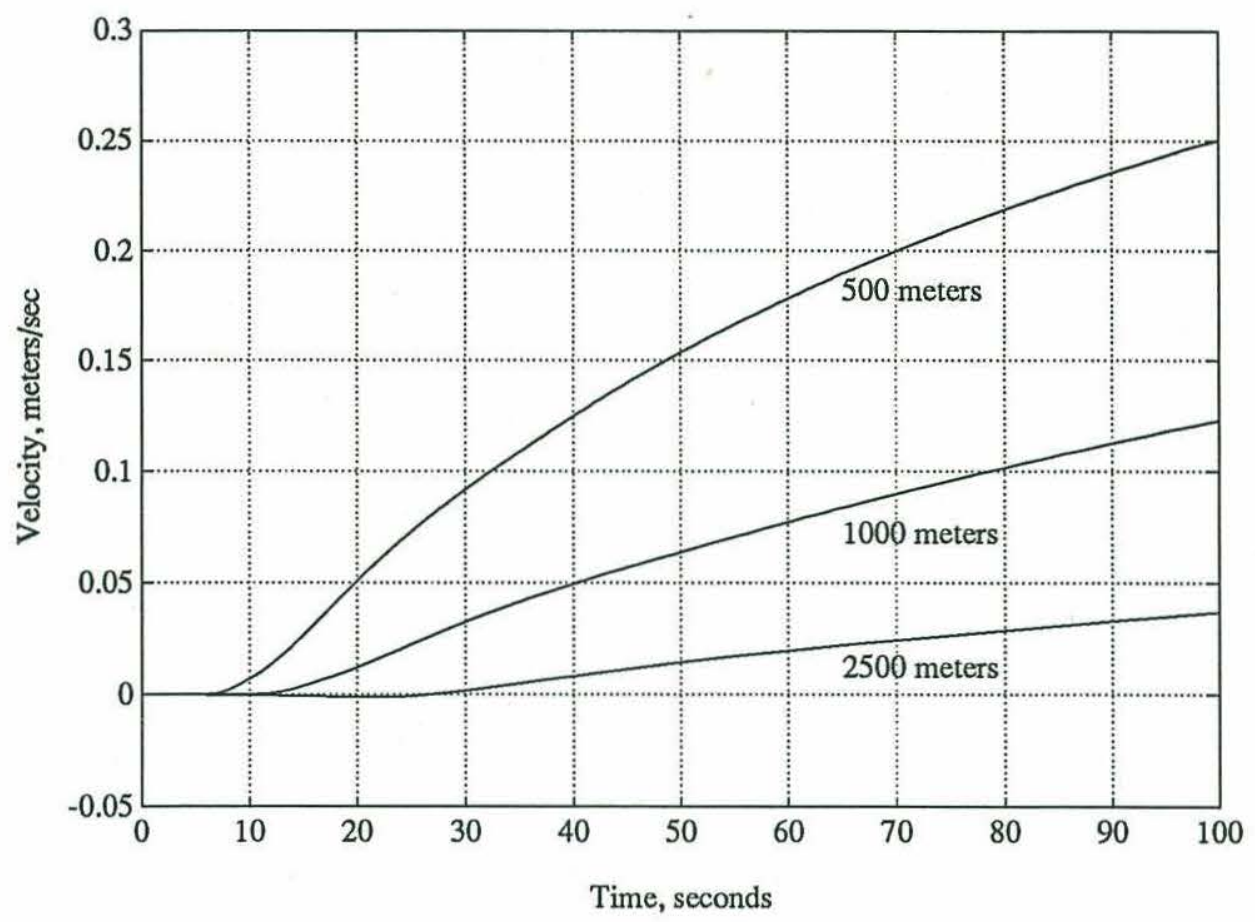

Figure 4-1: Simulated Step Response vs. Cable Length

In addition to the effects of cable length on response speed, we can make similar statements about the role played by vehicle weight. Figure 4-3 shows responses to the step of the previous example, with a 2500 -meter cable and varying in-water vehicle weights. In general, a heavier vehicle is seen to reduce the pure time delay, and to quicken the system's gravitation toward steady state. The pure delays approximated using the wave speed of above are 20.2, 17.7, and 15.8 seconds for weights of 6000 , 11000 , and $16000 \mathrm{~N}$, respectively; this agrees fairly well with the corner points of the figure. After the delay, although a larger in-water weight implies a larger bottom mass, and perhaps a slower velocity growth due to inertial effects, usually the change in catenary restoring force is much more important, and the response speeds up regardless. (Interestingly, a quick comparison of Figures 6-2 and 4-2 further exemplifies the effect of vehicle weight: the $19000-\mathrm{N}$ ball on 1200 meters of cable has a response quite similar to the 6000-N MANGUS vehicle on only 740 meters.) 


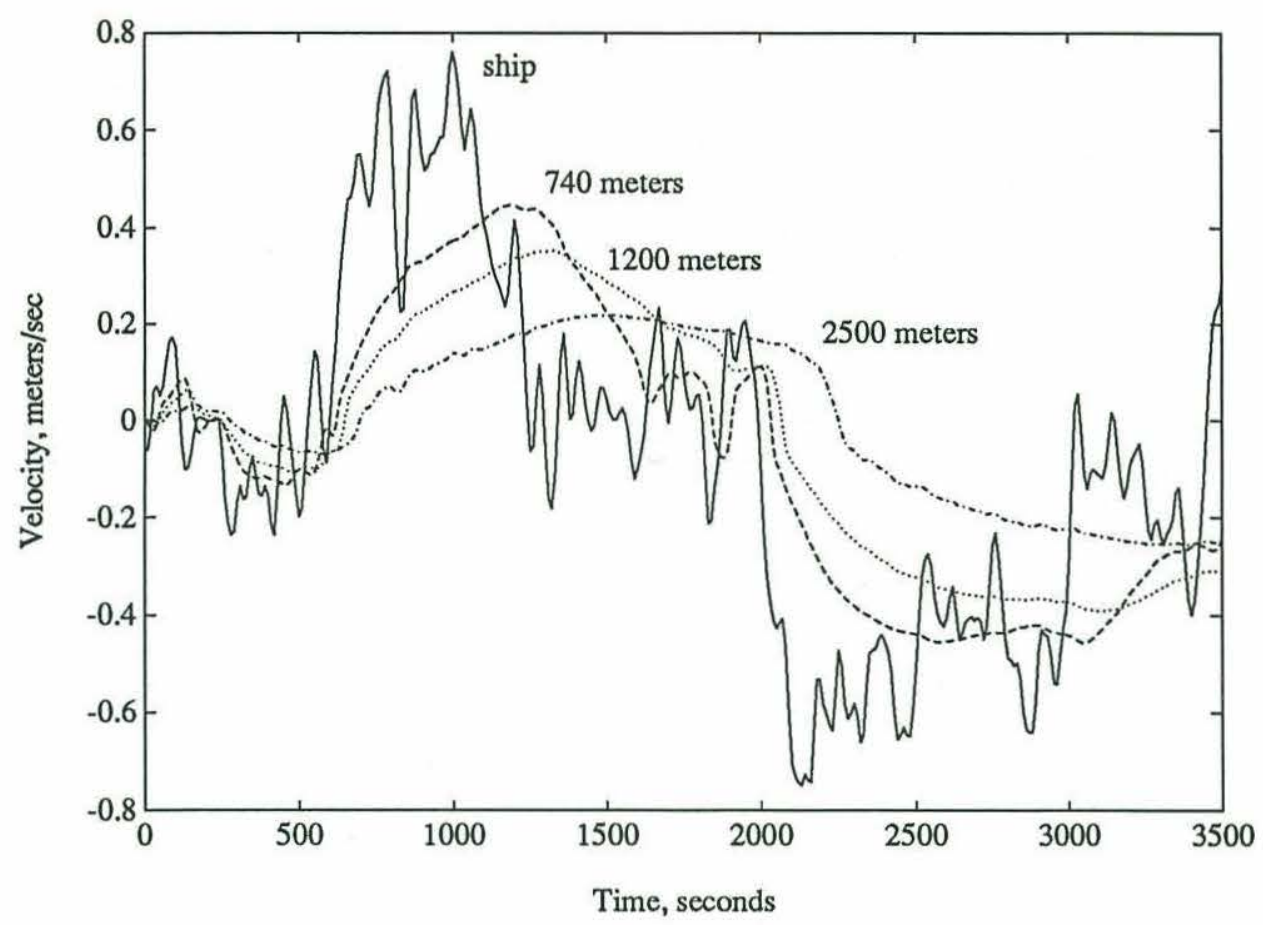

Figure 4-2: Effect of Cable Length on Simulated Vehicle Response

Thus, minimization of cable length is one strategy by which the ship-following capability of the system can be enhanced: unfortunately, this length is typically dictated by the operating depth and the necessity of being close to the seafloor, not by the desired horizontal dynamics. Increasing the system weight in water is the other avenue to consider; changes here are more readily effected through system design and assembly. However, there are hard size limits for all configurations, mainly due to deck-handling and deployment considerations. For the most part, the ARGO/JASON system weight has been maximized within these bounds. 


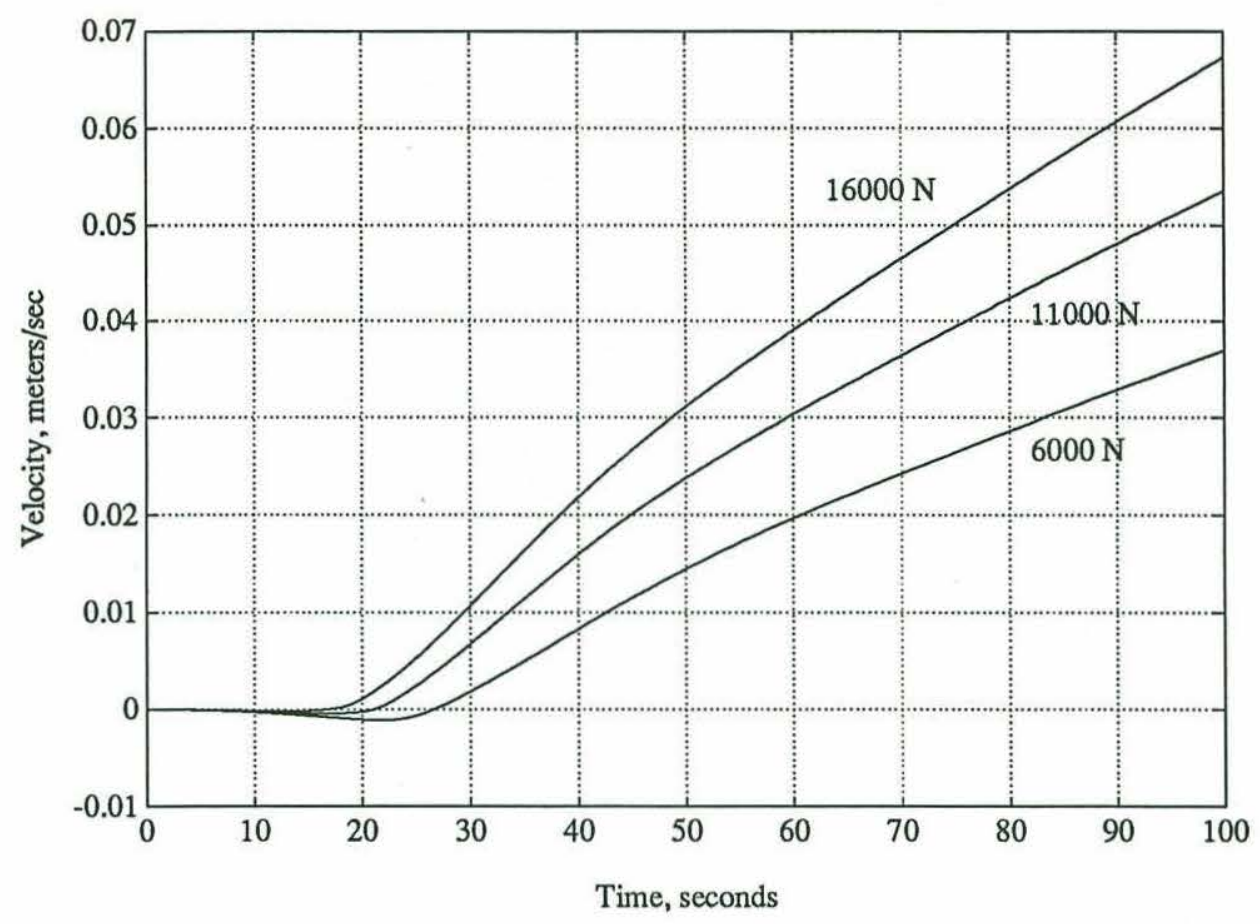

Figure 4-3: Effect of Vehicle Weight on Simulated Step Response

\subsection{Settling Time and Optimized Ship Motions}

It can be shown that significant improvements in system performance are obtainable through relatively simple controller tactics, emanating directly from a basic description of the dynamics. For example, consider a point-to-point maneuver in which the objective is only to move the vehicle from its current location to a new one as quickly as possible. For the ARGO/JASON system, this is a standard exercise during exploration operations, in which JASON may move randomly between different sites of interest on the seafloor.

The simplistic, conservative approach to the problem is to move the ship over the new location and wait for the vehicle to settle underneath; this is illustrated in Figure 4-4 for a 100 -meter excursion and a ship velocity of .5 meters/second. Here, our view is from the 
side, and snapshots of the cable shape are taken every twenty seconds. As before, the pure time delay and formation of the catenary are the important factors in startup at the vehicle. The figure also shows the cable evolution after the ship has stopped; here is one of the fundamental frustrations encountered when executing point-to-point trajectories in this manner. The final state shown is fully ten minutes after the start of the trek, and six-and-a-half minutes after the ship has stopped over the desired location, yet the vehicle is still twenty-seven meters from the goal, and slowing. Clearly, a good deal more settling time is needed in this case to get the vehicle sufficiently close to the goal. In general, for a long cable length, these waits may become excessive (thirty minutes or more) if the strict point-to-point ship path is maintained.

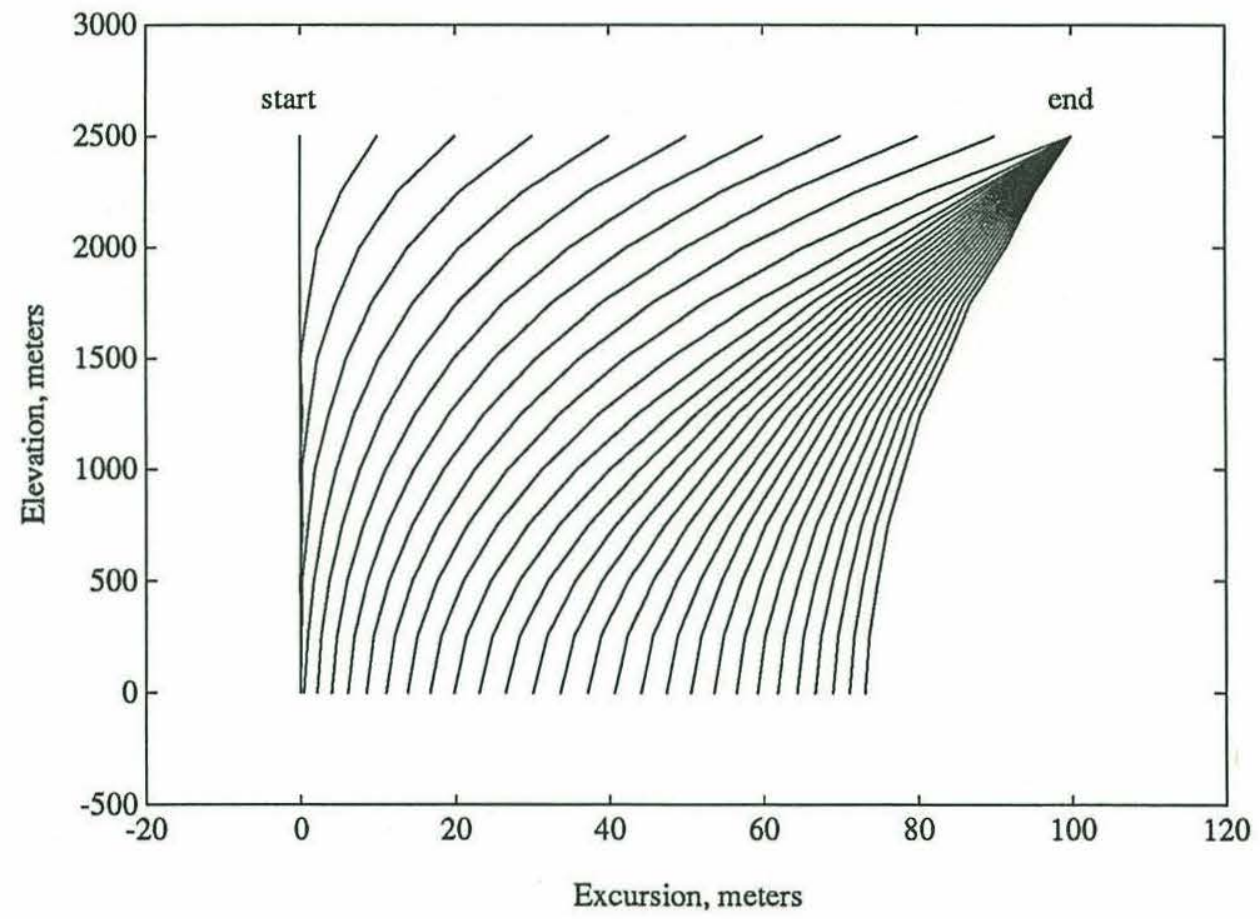

Figure 4-4: Cable Evolution in Direct Point-to-Point Maneuver

Fortunately, we can now take advantage of a system model for this problem, and investigate alternative ship trajectories. An obvious choice for a first method is to 
overshoot the final location with the ship, and then retrace the track backwards. This will allow us to cut short the slow falling of the catenary. An extension of the above example is shown in Figure 4-5, where for a 100-meter excursion, the ship overshoots the goal by another 100 meters, and then comes directly back to it. The catenary has been actively eliminated in this maneuver, and after the same ten minutes of elapsed time the vehicle is to within seven meters of the goal.

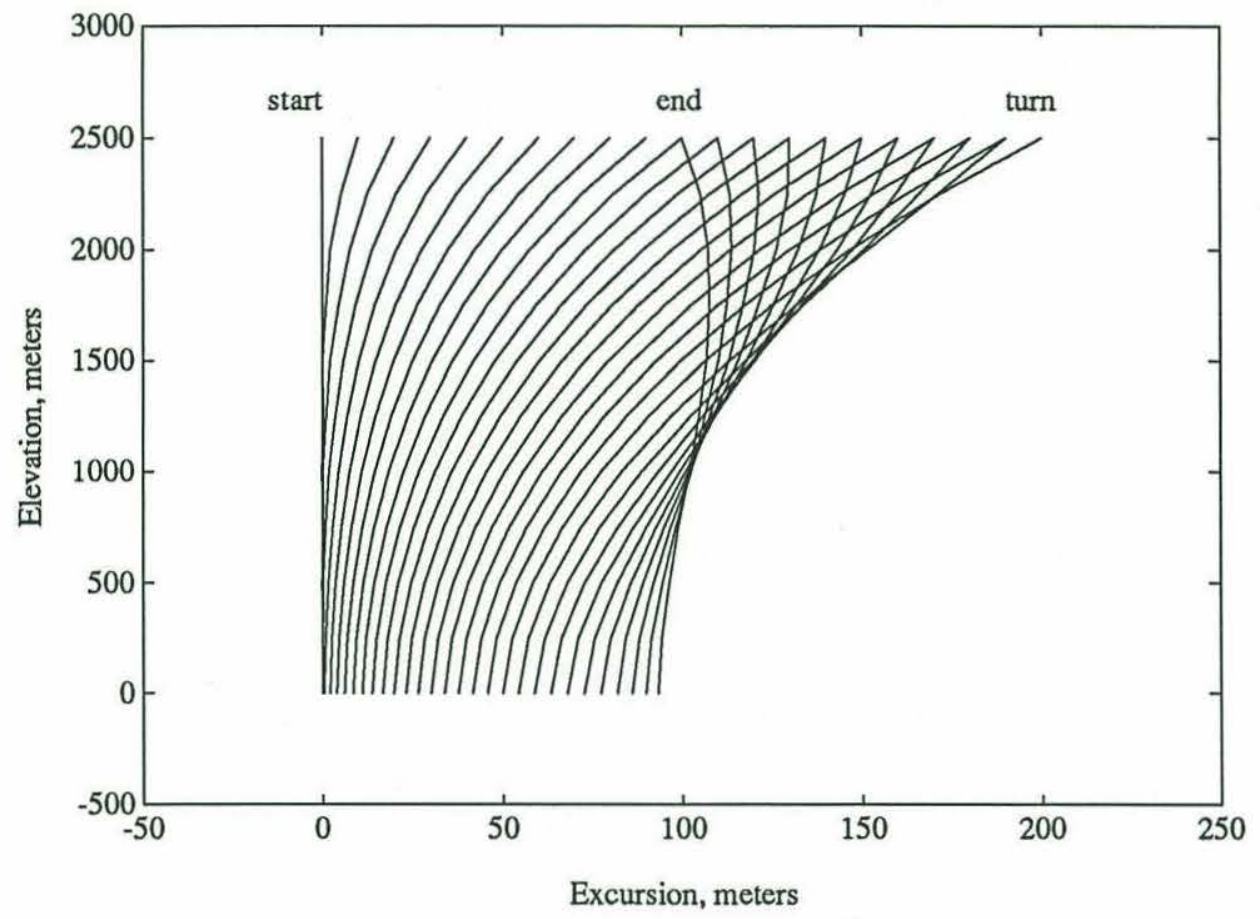

Figure 4-5: Smart Point-to-Point Maneuver

While this example is by no means the only answer to the problem of optimal vehicle placement, or the best one, it does illustrate a tangible capability to enhance the performance of the system during typical maneuvers. In fact, this overshoot/retreat rule was utilized in an open-loop sense during a recent cruise; a significant reduction in settling time was observed. In the future, these ship tracks will ideally be dictated by the controller itself, provided that the system model is able to correctly predict the behavior, 
$-56-$

and that the controller is "smart" enough. 


\section{Chapter 5}

\section{The Identification Method}

As pointed out in Chapter 1, we find that the analytical spectral model has several significant shortcomings. Namely, to propagate the system requires a large computer budget, and only the fastest processors in a dedicated integration loop can achieve realtime solutions. Furthermore, complete control of such a system (in the modern controls sense) would require an infinite number of states. We now seek a condensation of this elegant solution, at the cost of physically-based parameters. That is, we must search for our parameters now, whereas for the analytical model, it was sufficient to specify the basic physical quantities.

\subsection{Analytical Model Use in Identification}

The primary goal in the sequel is to establish that the spectral model can generate inputoutput sets which may be used a priori to create parameterized dynamic models that adequately describe the actual physical system, without requiring any real sea data. An overall identification plan for this is described in Figure 5-1. Given knowledge of the basic physical parameters (length, diameters, drag coefficients, masses, etc.), the first step is to use the analytical model to find the time series response to a typical ship input. This input is governed in velocity by the limits recognized for a particular operation, normally under one knot. Its frequency content is bounded by the bandwidth of the dynamic positioning system and the ship's response to waves. Further, in light of the cable shapes encountered in Chapter 4, it is prudent to include some regions of zero-mean speed as well as some of a steady-state tow speed.

For the purposes of a priori identification, the above analytical model results are taken as 


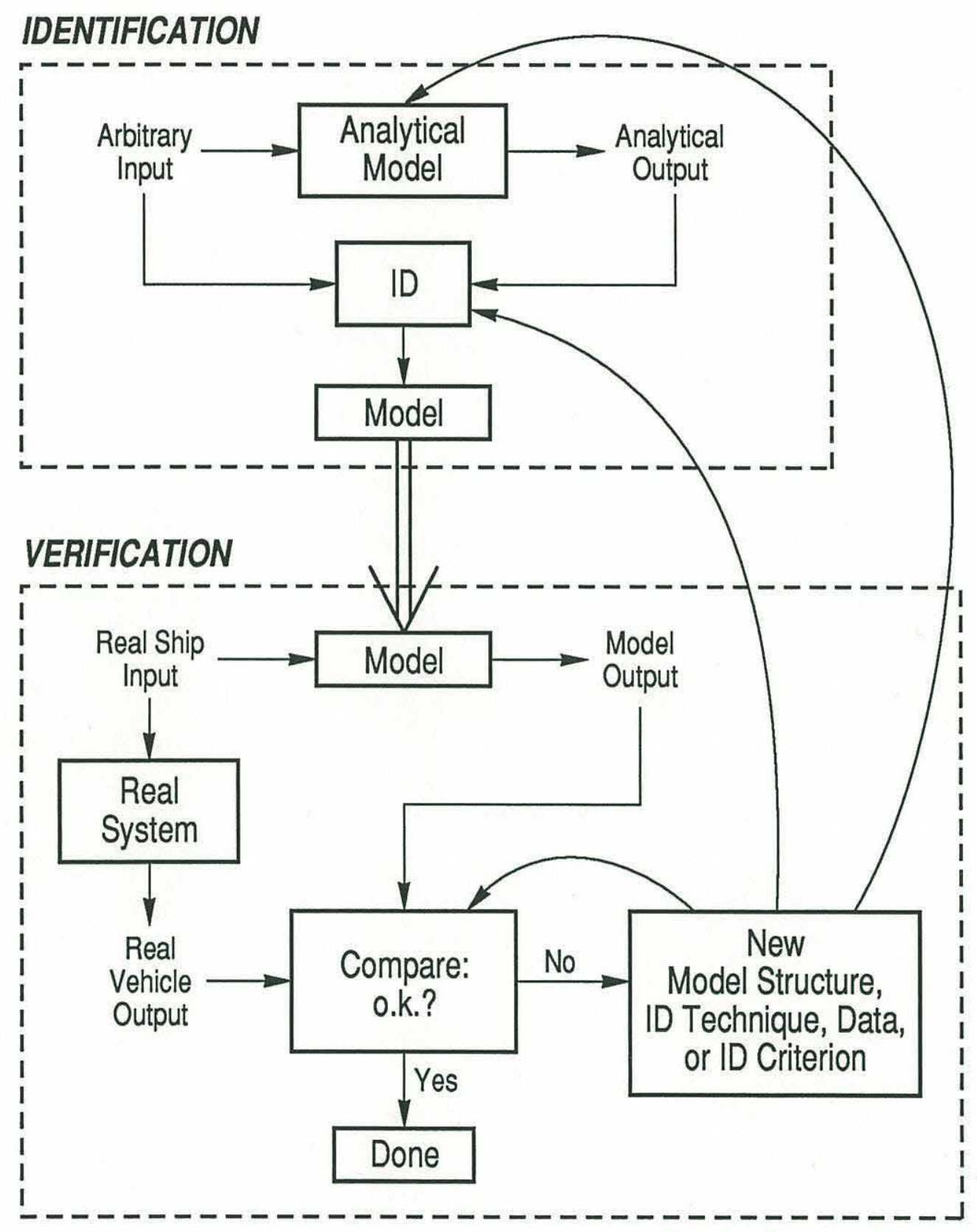

Figure 5-1: Overall Identification and Verification Method

truth. While real data could be used in identification, the advantages of the analytical 
"truth" are that it is noise-free, and, as stated earlier, systems can be identified for which there is no real data yet. From this, once a model form has been specified, and a method by which to identify the parameters has been adopted, the parameterized form is developed. A later section in this chapter will address the current technique.

Verification of the identified parametric model is obtained by applying input from real sea data. Propagation of the short model is relatively quick, and we can directly compare this result with the real response of the vehicle in the ocean. A measure of the "goodness of fit" is taken, and a decision is made as to whether or not the result is satisfactory. In the event that the identification has failed, new model structures, data sets, or new identification techniques or criterion are all reasonable modifications. Parts of the whole process in Figure 5-1 are repeated as necessary.

\subsection{Model Identification Method}

The literature on system identification and parameter estimation is replete with various methods for accomplishing a task such as ours. Due to the nature of the system, however, the linear standards such as step responses, frequency analysis, and ARMAX models have had little success, as one would expect. Batch processing least-squares techniques are difficult unless good measures of all states and their derivatives are available. Instrumental variables applied to the basic least-squares technique can solve some of this, but have not been looked at in detail. On a similar note, an Extended Kalman Filter for state and parameter estimation has been used with some success for time-varying models, but those results are outside the scope of this work. Some insight into these techniques is given in [Ljung 87] and [Gelb 79]. 


\subsubsection{Learning Model Method}

Learning Model Methods are described in [Sage 71], and are most simply described as minimizations of the error in the output variable in an open-loop simulation sense, based on iterations in which more and more is learned about the system. For the current application, the problem is to minimize the overall simulation error in the vehicle state given by the model, which is driven by an arbitrary input series. A flow graph for this learning process is shown in Figure 5-2.

This method is extremely flexible; any system which can be simulated ideally can be identified. In addition, it is particularly well-suited to systems corrupted by noise due to sensors or to a differentiation process. Noise in the input is, in general, filtered by the dynamics of the model and, conveniently, noise in the output of the real system can be filtered using simple techniques, only as necessary for comparison with model output. One disadvantage of the learning-model approach is that a full simulation is required for the computation of each error measure; thus, an iterative search technique may take some time to converge. The error measure is here taken as the uniformly-weighted root-meansquared error.

In the simulations, there are no corrections when measurements are available, and thus, for continuous systems integration accuracy may become an issue. The problem of global error is further exacerbated in higher-order systems, which may have stiff modes. The adaptive stepsize Classical Runge-Kutta integrator is described in detail in [Press 87], and is ideal for this application. In essence, the stepsize is controlled to maintain (at least) the desired accuracy for all states. 


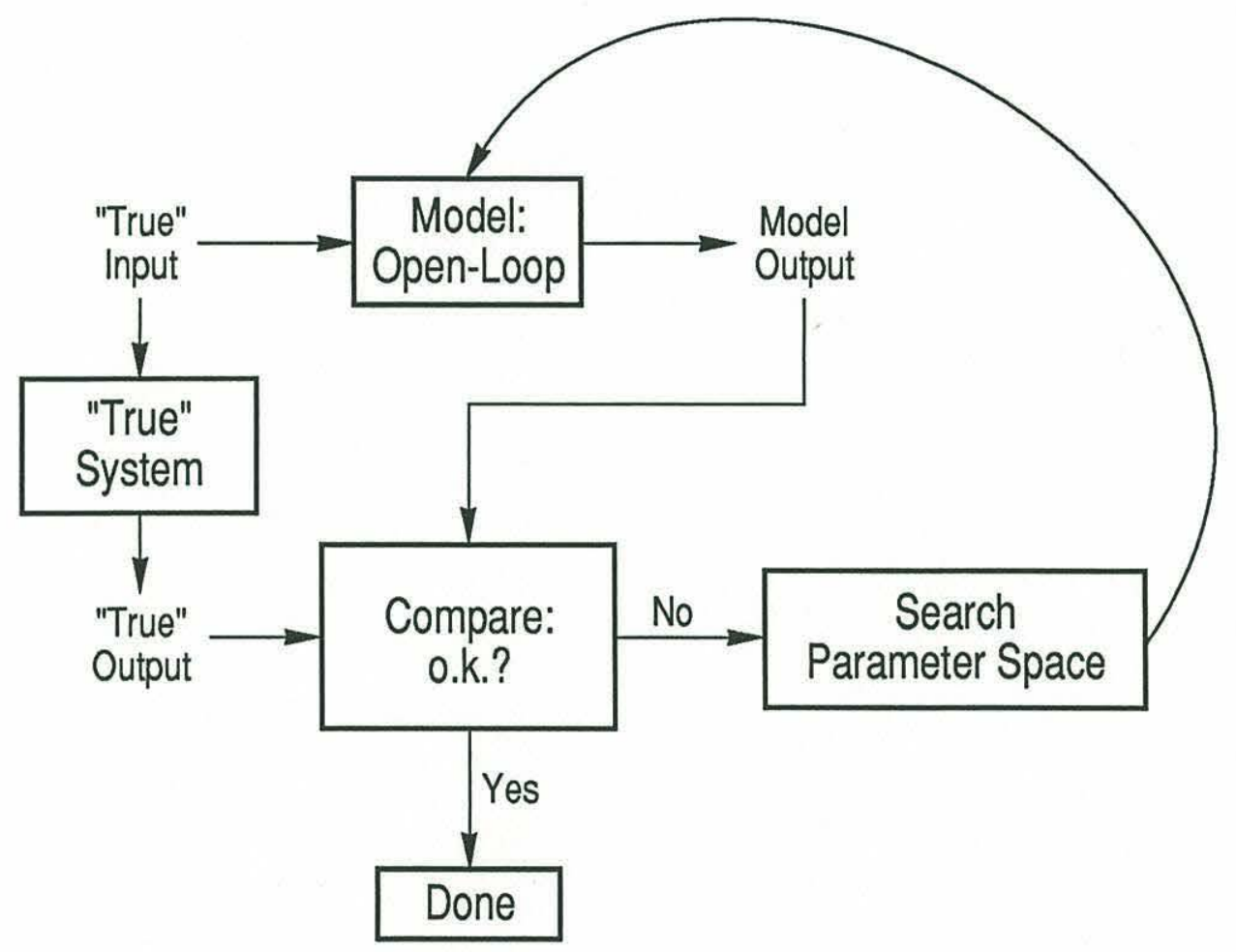

Figure 5-2: Learning-Model Method Flow Graph

\subsubsection{Nelder-Meade Simplex Algorithm}

The search for the minimum rms error in a simulation made from $\mathbf{n}$ guessed parameters is a search for the minimum of a certain function in $\mathbf{n}$-dimensional space. Conventional gradient methods are not easily applicable here because they require evaluation of the function's derivatives, something we are not able to produce. Here, we have chosen the Downhill Simplex method of Nelder and Meade; it is described in [Press 87]. A simplex is a geometric entity in the $\mathbf{n}$-dimensional space, consisting of $\mathbf{n}+1$ vertices. For instance, a search in two parameters implies a triangular simplex (see Figure 5-3(a). 
Through a series of carefully worked-out steps, the ameoba-like simplex is supposed to move in the downhill direction, eventually ending up at the (sometimes local) minimum. Interestingly, the simplex can stretch out to get across "smooth terrain" quickly, yet can shrink to nearly infinitesimal size to get through the narrowest of "canyons". The four possible steps are shown in Figures 5-3b) through 5-3e). They are: b) a reflection away from the highest point, c) an expansion away from the highest point, d) a contraction from the highest point, or e) a contraction toward the lowest point.

This pattern requires no evaluations other than that of the function itself. As a result it is generally quite robust, although expensive in evaluation time. In this work, the "nelder" function provided with the PC-MATLAB [The Math Works 88] environment was used, and a restart performed upon completion to ensure that the search had not ended on a local minimum.

We are now ready to specify the model structures of interest, and to carry out the identifications as detailed above. Criteria for the success of the models have not been rigidly defined; graphical comparisons of the models provide some distinguishable merits, but emphasis is given to the rms errors in position and velocity. 
$-63-$

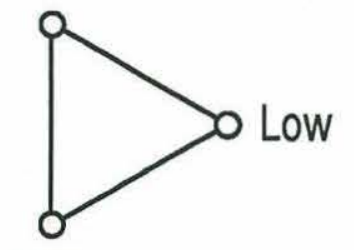

High

a)

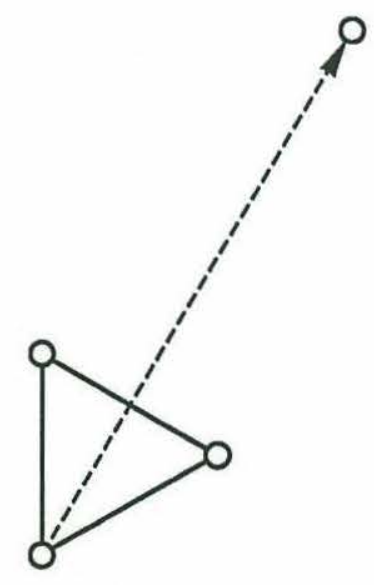

c)

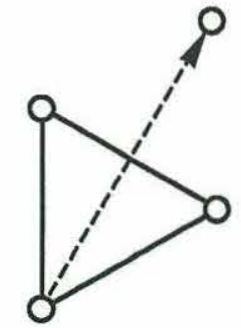

b)

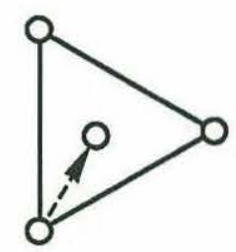

d)

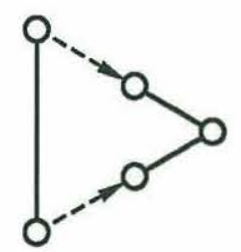

e)

Figure 5-3: Nelder-Meade Simplex Algorithm 


\section{Chapter 6}

\section{Model Forms and Results}

Emphasis was placed in Chapter 3 on the ability of the analytical model to simulate real sea data with a minimum of parameter tuning. The current chapter utilizes the model to create parameterized forms suitable for control with the available methodologies; we seek sets of differential equations with possibly quadratic drag and explicit time delays. Verification of these short models is achieved by subjecting them to actual ship inputs, and comparing the results with the actual vehicle responses.

\subsection{Ship Trajectory for Identification}

For simplicity, a single fictitious ship trajectory has been used to drive the analytical model in all three scenarios. The input here is shaped by two real-world parameters; velocity amplitude and bandwidth. The amplitude is generally bounded to something under one knot, a typical upper operating limit for deep deployments. The input's frequency content can be determined from the spectrum of an actual ship track; Figure 6-2 shows the spectrum from a 2500-meter Tyrrhenian Sea run, as an example. Based upon this graph and similar consideration of other data sets, a cutoff for the ship was chosen at .06 radians/second. This cutoff likely encompasses higher-frequency wave and wind effects, at bandwidths beyond the capabilities of a typical dynamic-positioning system.

In this work, the final input was created by first generating a random series of velocities with normal distribution. Since nonzero speed ranges are suspected to be an important component of the identification input (because the catenary restoring forces are not linearly related to the deflection), arbitrary biases were then added to parts of the random 
sequence. Next, a second-order Butterworth Filter was used to exert the bandwidth limit on the random sequence, and finally, the entire set was scaled as necessary to match the imposed velocity limits. The velocity series used for identification is shown in Figure $6-2$, along with the simulated responses of the the three systems of interest.

As noted in Chapter 4, the fact that the heavier steel sphere on 1200 meters of cable has a response very similar to the MANGUS vehicle on 740 meters evidences the utility of a greater vehicle weight in water; we have made the cable look shorter and have sped up its dynamics.

\subsection{Real Sea Data for Verification}

For the real data sets prescribed in the previous chapter, runs were used from the Tyrrhenian Sea with MANGUS on 740 meters of cable, the same AUTEC run that was used to verify the analytical model (steel ball and 1200 meters of cable), and one of the

runs from the Tyrrhenian Sea with MANGUS on 2500 meters of cable. The velocity and position records for these cases are shown in Figures 6-3 through 6-8.

These three runs cover a variety of scenarios, apart from the differences in vehicle shape and weight, and cable length. First, the 740-meter run consists of starts and stops in one direction only, with velocities of a single sign--these are variations about a tow speed of approximately .25 meters/second. The AUTEC data is richer in frequency content, and contains especially sharp transients, as well as regions of steady speed. Finally, the Tyrrhenian Sea data with 2500 meters of cable has a small transient at the start, but for the most part has only several distinct frequencies and amplitudes. Although an abundant frequency content is usually desirable, data such as this may involve the wave-carrying properties of the cable more than the other cases; that attribute of the physical system is significant. 
$-66-$

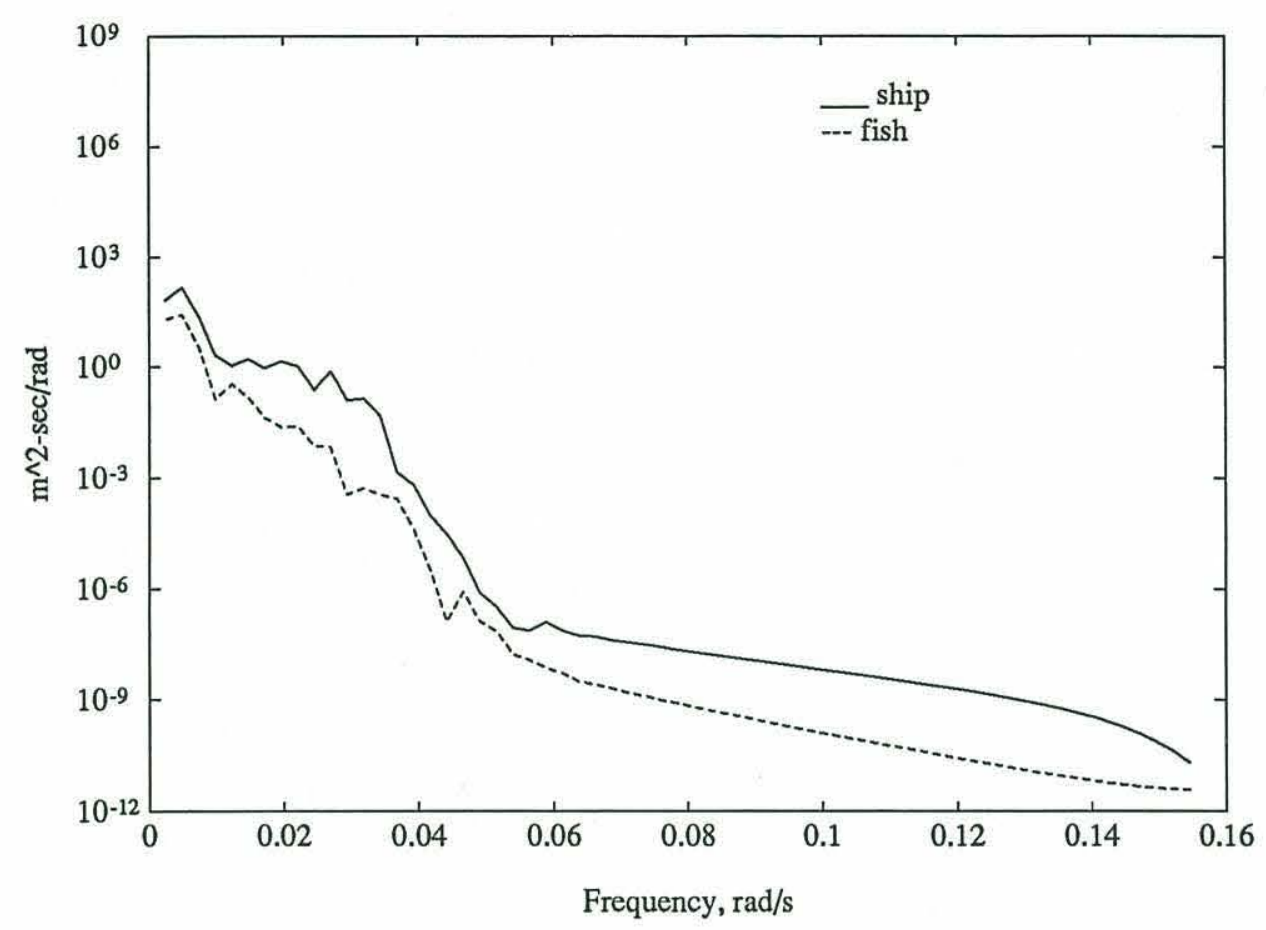

Figure 6-1: Input/Output Spectrum for a 2500-meter Experiment

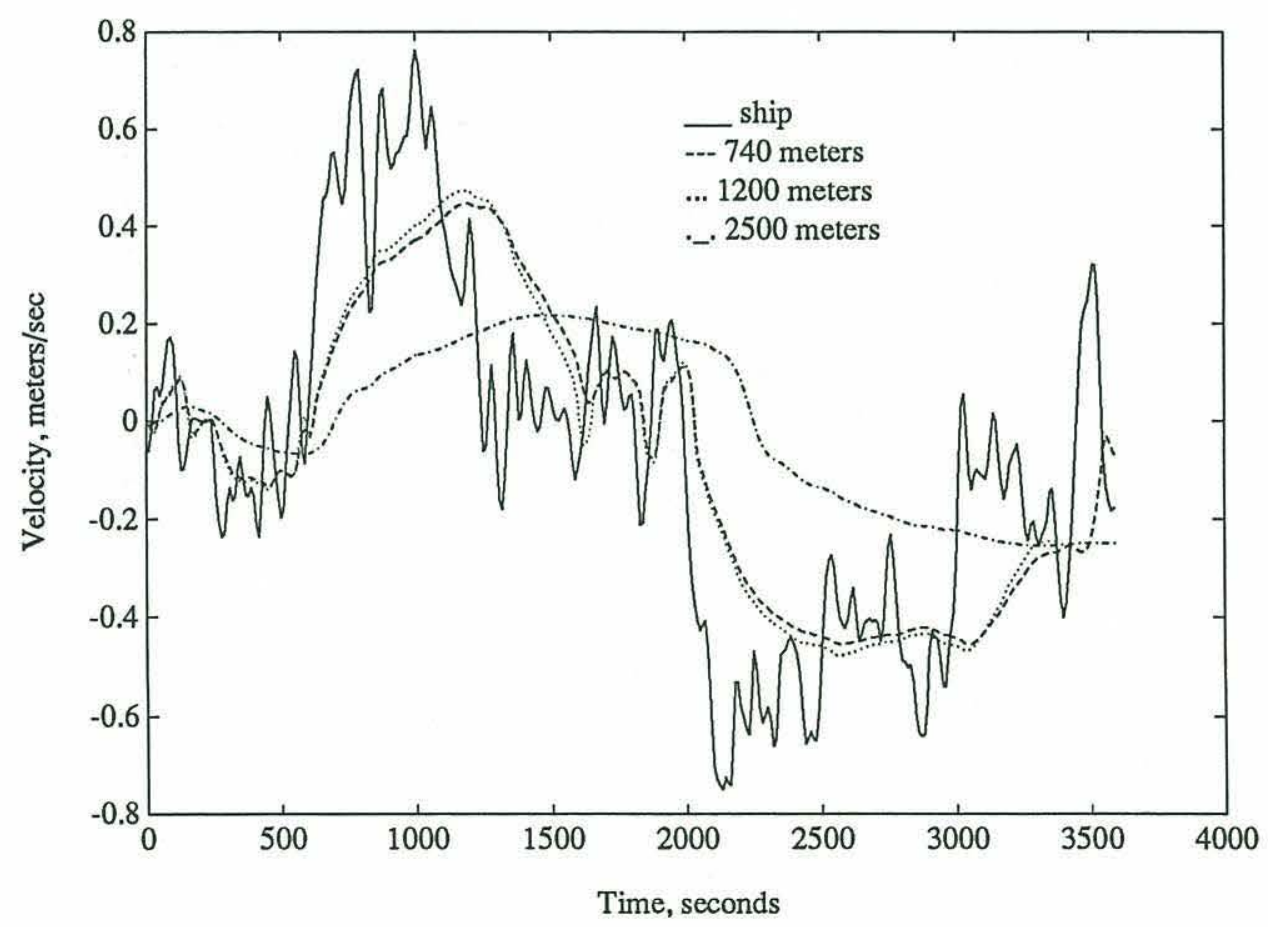

Figure 6-2: Identification Ship Velocity Series and Responses 
$-67-$

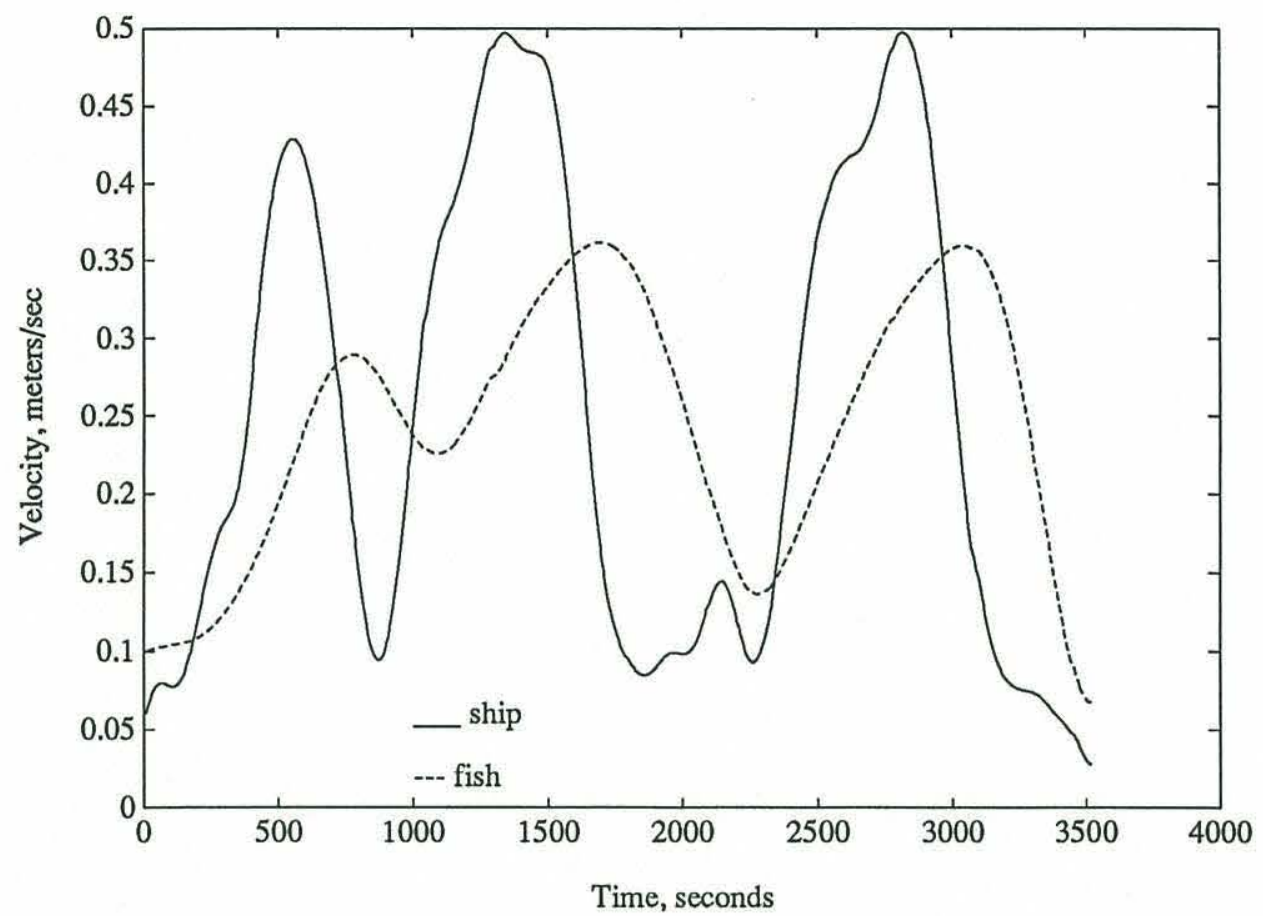

Figure 6-3: 740-meter Experimental Data: Velocity

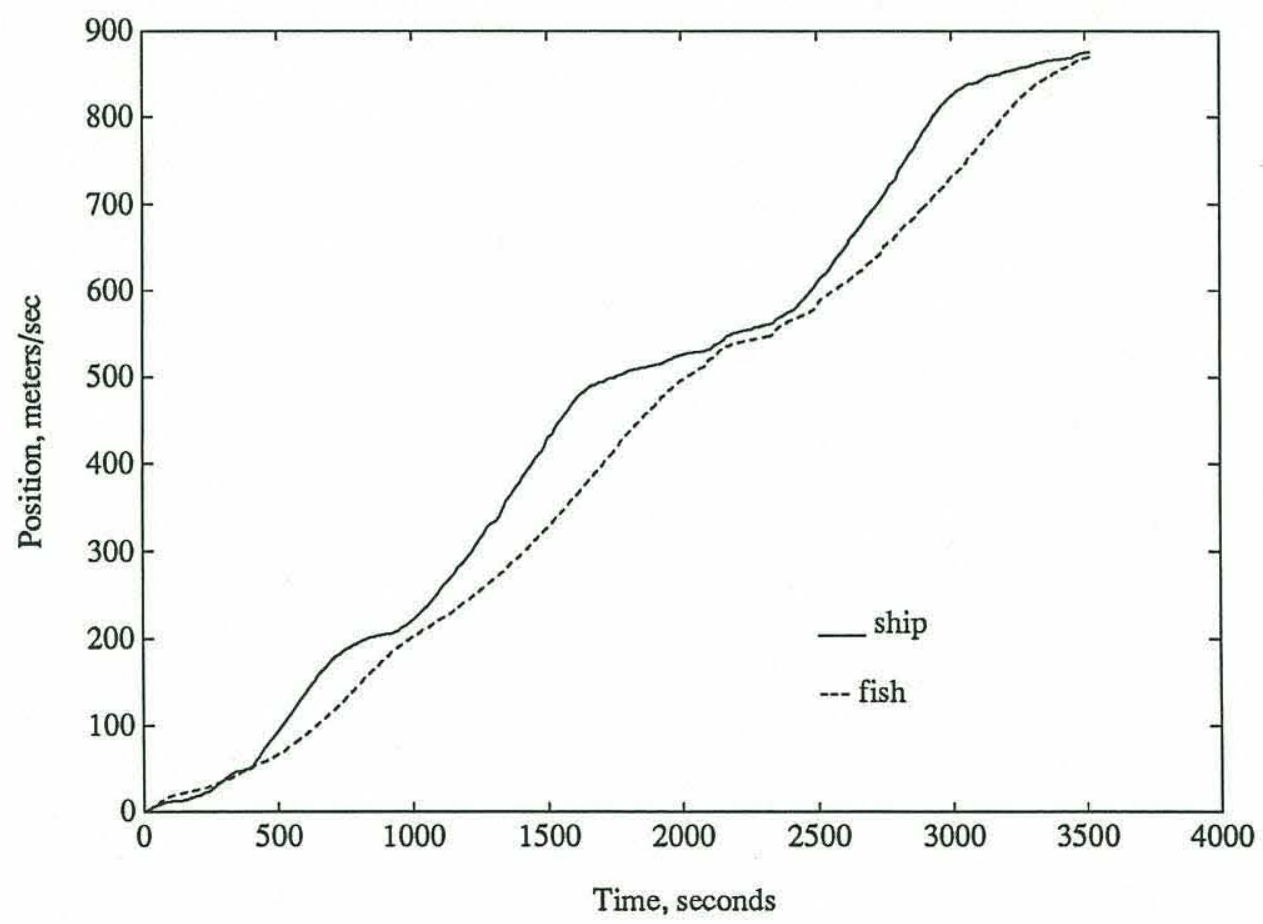

Figure 6-4: 740-meter Experimental Data: Position 
$-68-$

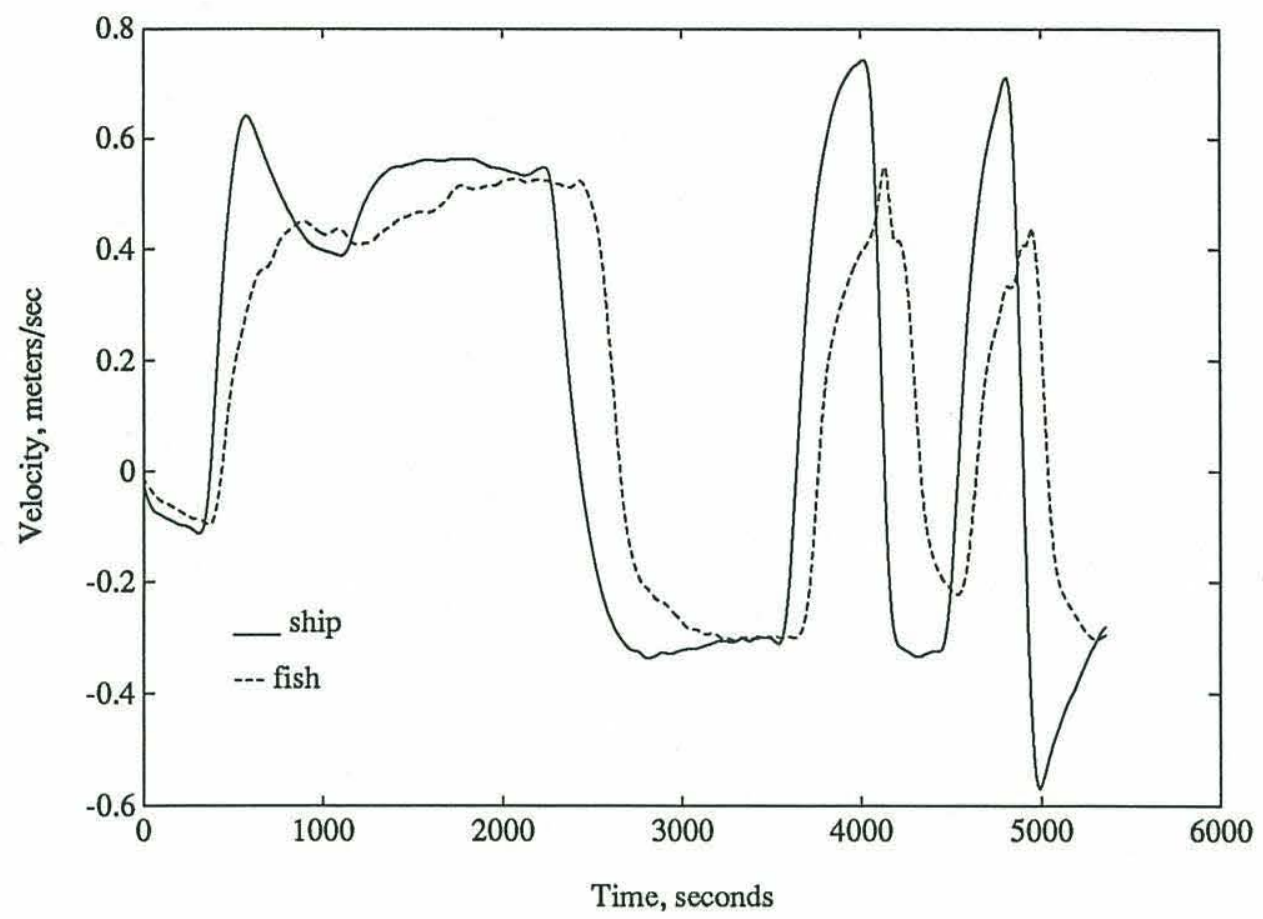

Figure 6-5: 1200-meter Experimental Data: Velocity

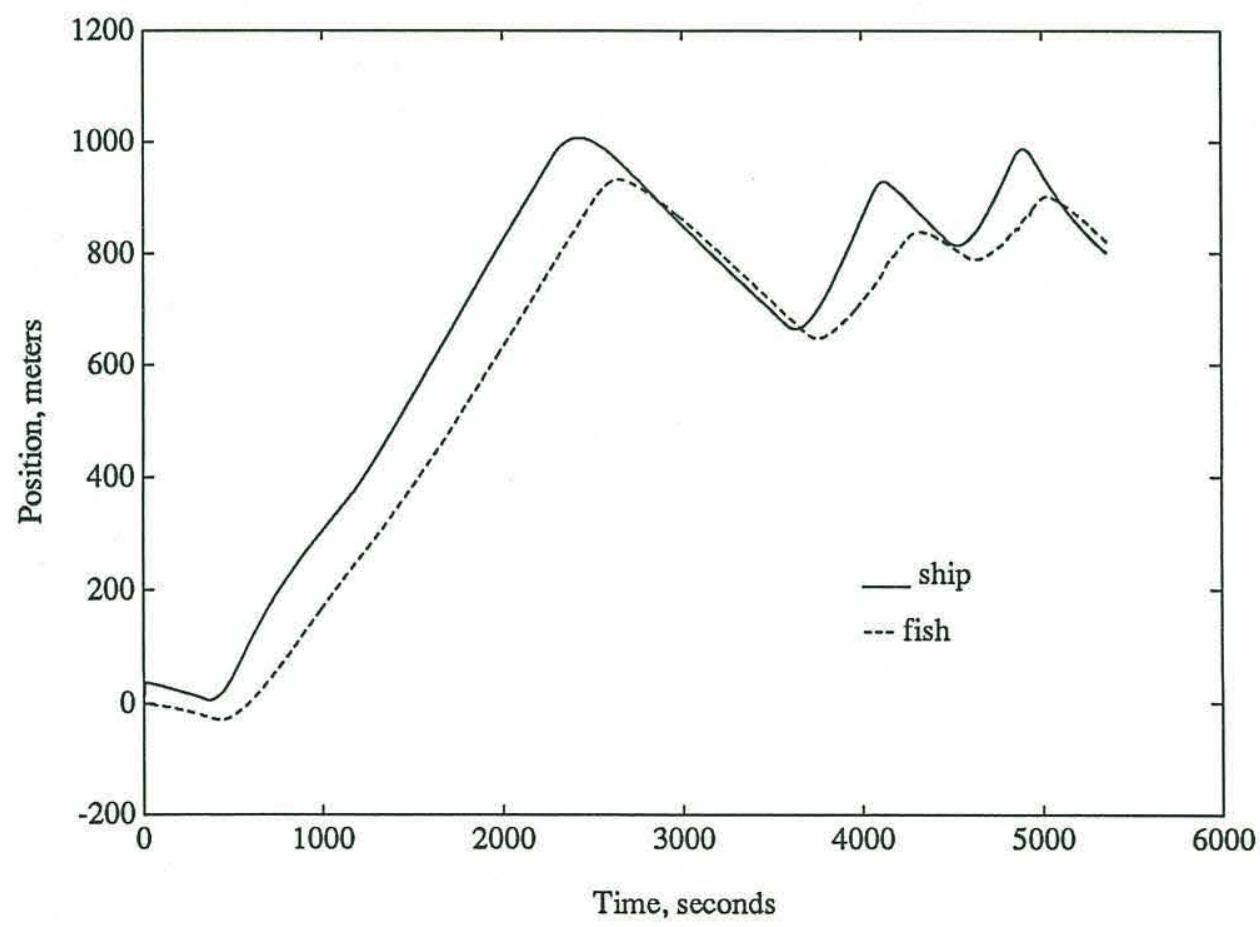

Figure 6-6: 1200-meter Experimental Data: Position 
$-69-$

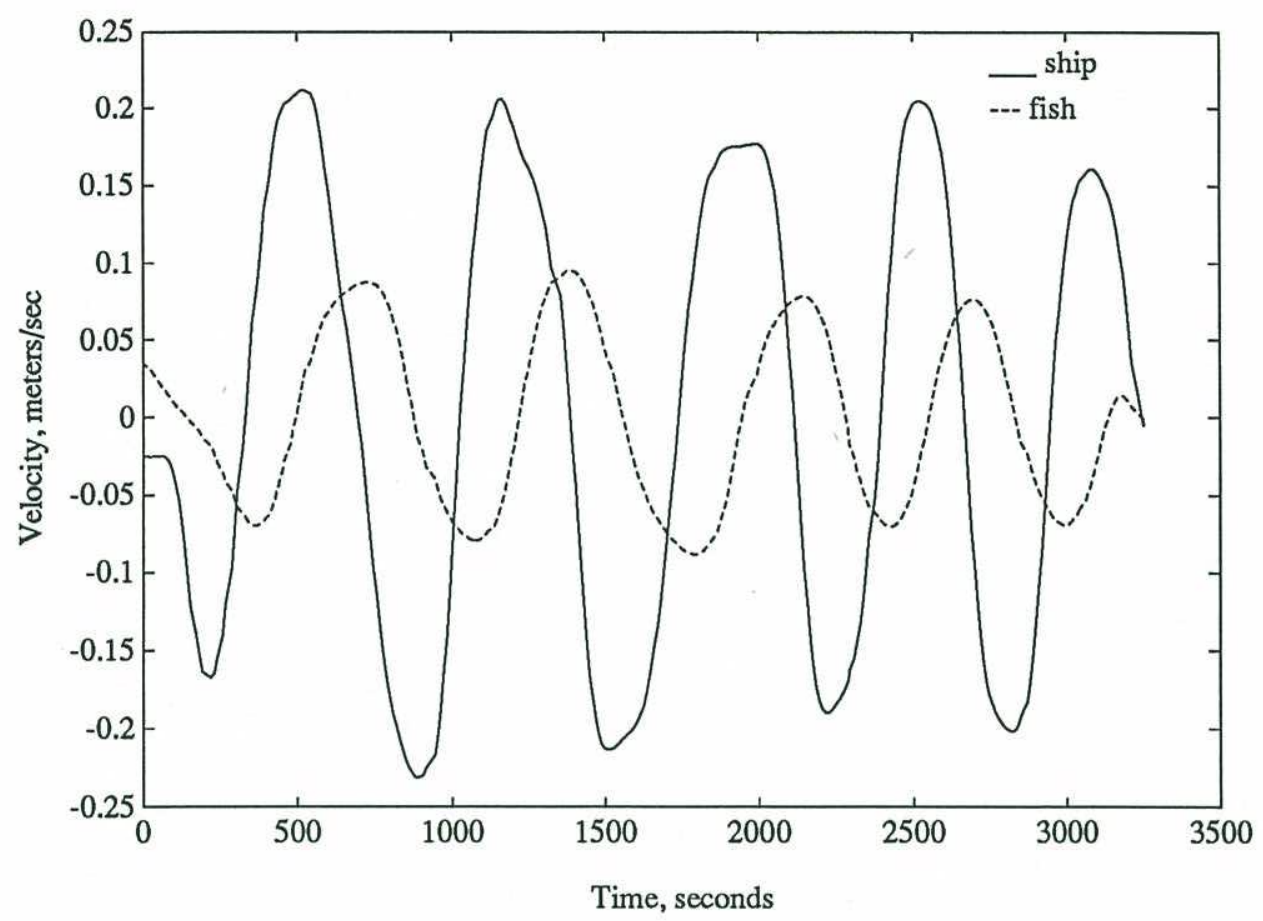

Figure 6-7: 2500-meter Experimental Data: Velocity

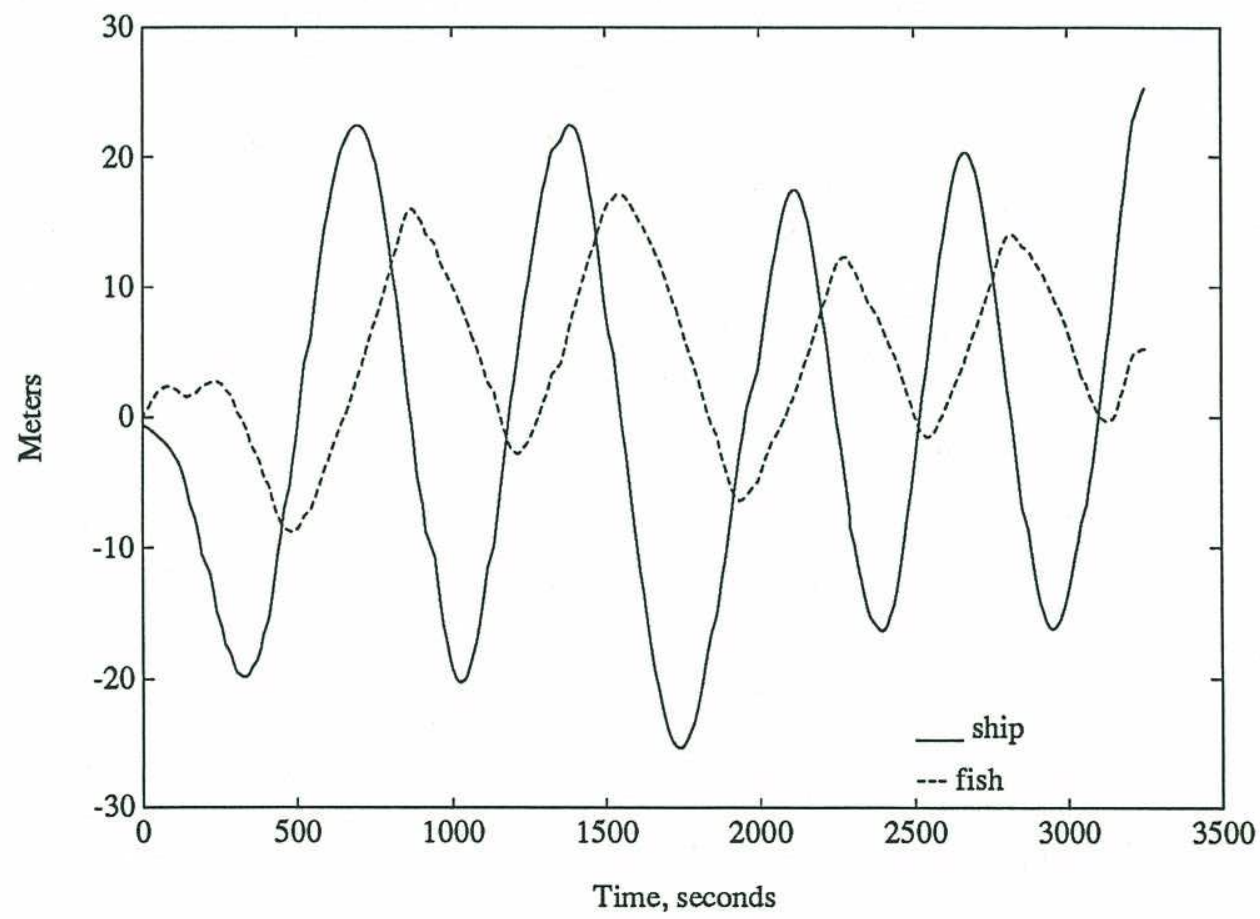

Figure 6-8: 2500-meter Experimental Data: Position 


\subsection{Second-Order Models}

Perhaps the simplest model form to begin with is that of the mass-spring-damper system. The physical equivalent of this system is shown in Figure 6-9, and the equations of motion for the linear and nonlinear drag case are:

$$
\frac{d^{2} x_{v e h}}{d t^{2}}=-\alpha\left(x_{v e h}-x_{\text {ship }}(t-\tau)\right)-\beta \frac{d x_{v e h}}{d t}
$$

and

$$
\frac{d^{2} x_{v e h}}{d t^{2}}=-\alpha\left(x_{v e h}-x_{\text {ship }}(t-\tau)\right)-\beta_{n} \frac{d x_{v e h}}{d t}\left|\frac{d x_{v e h}}{d t}\right|
$$

Here, $\alpha$ is the "spring constant", $\beta$ and $\beta_{n}$ are the linear and nonlinear drag multipliers, and $\tau$ is the time delay as found in Chapter 4, and applied explicitly.

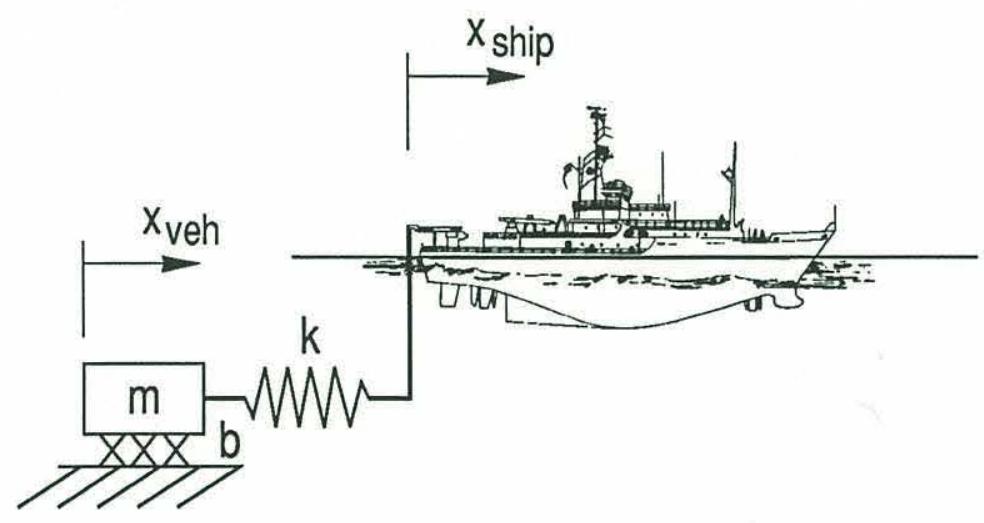

Figure 6-9: Second-Order System 


\subsection{Higher-Order Models}

A further sophistication is the decomposition of the cable into discrete points, each of which is a distinct mass and drag source; intrinsically, this form should be able to model effects such as time delays and lateral waves, which were unnatural with the secondorder models above. At the same time, if the order can be kept within reason, its candidacy as a controller model will be ensured. An $\mathbf{2 n}$-dimensioned system is shown in Figure 6-10; it should be noted that the vehicle dynamics are an integral part of the model regardless of the order, and that they alone constitute two states.

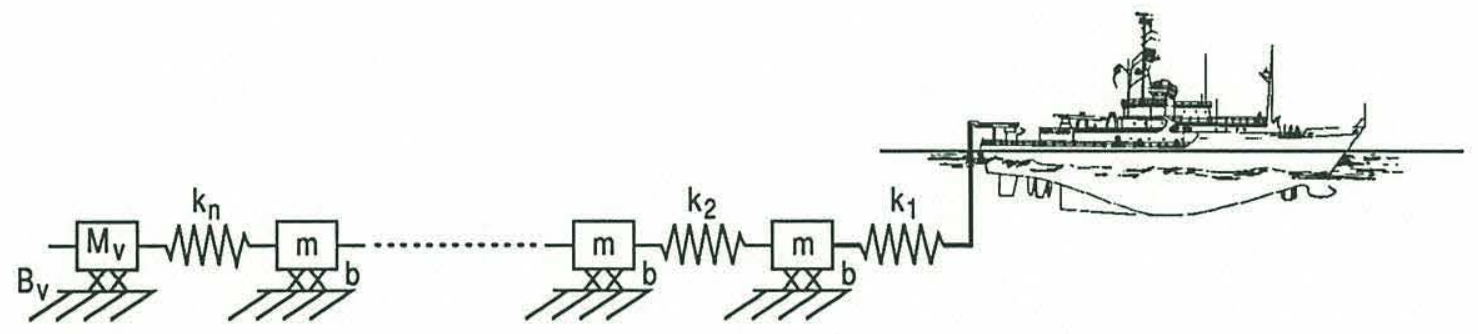

Figure 6-10: Higher-Ordered Cable Model

Tension is not assumed constant along the cable but is determined locally by the weight

of the vehicle and cable in water. Thus, if the deflections are small compared to $d s$, and the small angle assumption holds, then the individual spring constants are given by:

$$
k_{i}=\frac{T_{i}}{d s}
$$


Through bond-graph modelling of the linear system, the following form is realized:

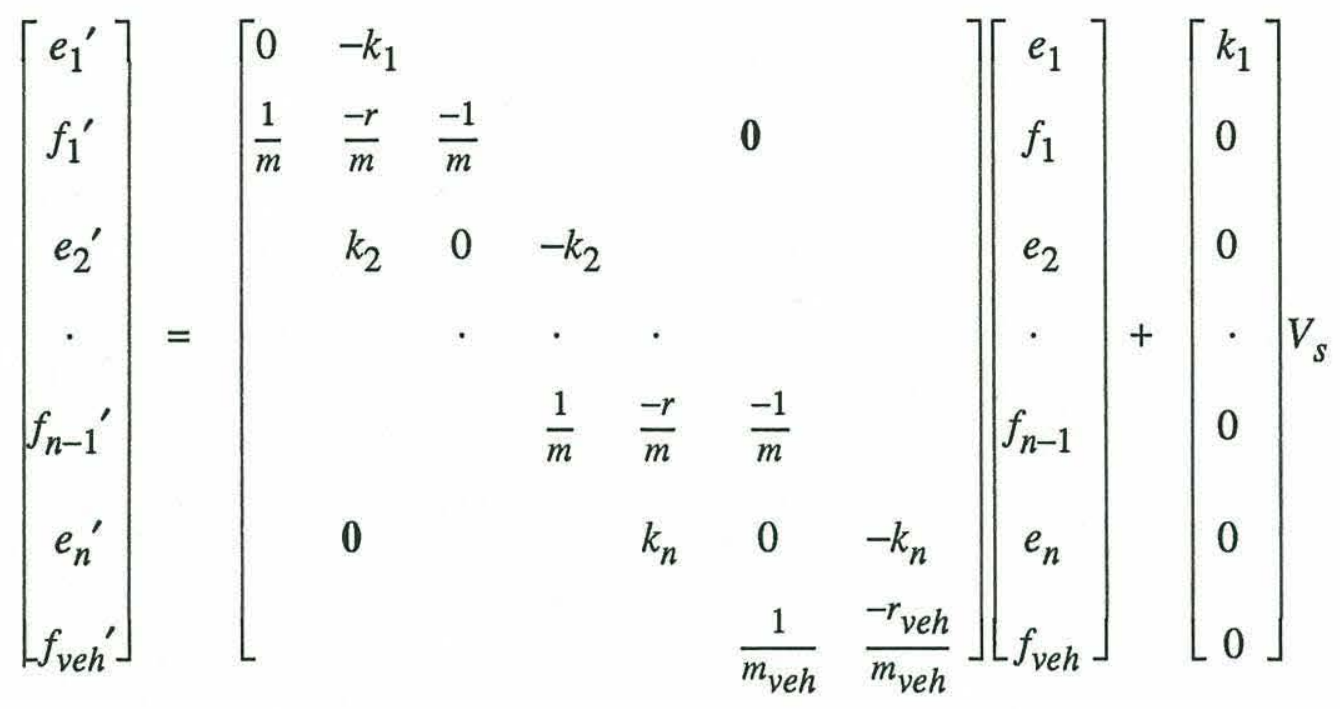

Inclusion of quadratic drag requires replacement of the terms $r$ above with $r_{n}\left|f_{i}\right|$, where $r_{n}$ is now the quadratic drag multiplier.

The second-order forms required two parameters. The new form will require only four, regardless of the number of links. Since the primary unknowns have to do with the drag and mass characteristics of the vehicle and cable, and the cable is assumed to have constant size and density, the unknowns are: 1) cable drag per node, 2) cable mass per node, 3) vehicle drag, and 4) vehicle mass. Intuitively, for increasing linkage resolution, the identified parameters of the link-based models approach those created by simply sectioning the continuous cable quantities (The solution approaches the finite-difference result). Thus, we should expect that educated initial guesses will not be too far off the final best-fit values; this nicety will be discussed in a later section.

The second-order forms were unable to model the pure time delay explicitly, but here the configuration of the approximated physical system can provide this capability, and it does not need to be specified. 


\subsection{Model Performances}

The criterion for fits between analytical model results and the above parameterized forms is taken to be minimization of the root-mean-squared velocity error, accepting the analytical results as "exact". However, we are also interested in the position error. Because states are typically unknown at the start of experimental data sets, the errors are computed only after approximate convergence of the simulation and actual velocity records. Of the higher-order models outlined, results are presented for the four-link case (eighth-order system), a choice which promises sufficient resolution, but still has a reasonable chance of realtime propagation. As mentioned in Chapter 5, integration of a high-ordered continuous-time system is best achieved through an adaptive-stepsize routine to satisfy accuracy requirements for the stiffest modes; these algorithms are quite expensive in processing time.

Four separate cases were considered for identification and verification: linear and nonlinear second-order forms with pure time delays, and linear and nonlinear eighthorder forms, from a four-link configuration. While it is recognized that the drag term is guaranteed to be quadratic, the attractive possibility that linear forms could suffice, giving much faster discrete-time propagation algorithms, warrants at least a casual glance at them. The Figures 6-15 through 6-26 in the Appendix give time records for the velocity and position errors, both for the artificial identification runs, and the verification runs from real sea data. More succintly, Tables 6-I and 6-II summarize the root-meansquared errors in velocity and position for all cases examined. Due to the diverse nature of each different input/output sequence, comparisons are made along rows, not columns.

As expected in the presence of quadratic drag, the nonlinear forms are clearly superior to the linear ones in all cases. The ratio of second-order nonlinear error to the minimum linear error ranges between about $63 \%$ to $110 \%$. The performance of the eighth-order 
nonlinear model is somewhat better; this ratio varies between $20 \%$ and $53 \%$. Thus, implementation of the nonlinear model with this data in general provides two to five times the modelling accuracy afforded by the linear models. At the same time, these results imply that significant gains are made by tolerating the computational inefficiencies associated with the higher-ordered models.

It is interesting to note that higher-order linear models are not necessarily better than lower-order ones; this stems from the fundamental inability for the linear drag term to describe the real process. Speculation on Table 6-I in fact shows that the second- and eighth-order linear models share about the same error characteristics. With this in mind, it can be recommended that if a linear model must be used, there is little to be obtained by increasing the order beyond two.

Comparison of the nonlinear second- and eighth-order forms is more definitive. The ratio of second-order to eighth-order model errors is between $22 \%$ and $72 \%$; in general, there is a factor of two or three to be gained in performance by using the higher-order form. The advantage of the link model is most visible in the results for the experimental 2500-meter run; in a regime where relatively fast oscillations are projected down the cable according to the modified wave equation (2.22), the ability of the model to exhibit some "travelling" wave behavior (that is, to approximate a differential equation in space and time) yields a significant improvement in accuracy. Again, however, the issue of computing speed is to be kept in mind, since the eighth-order form is subject to more stringent integration dynamics than the simpler model. This difference has been seen to account for several orders of magnitude in runtime. 


\begin{tabular}{|c|c|c|c|c|}
\hline & $\begin{array}{l}\text { Linear } \\
\text { Second- } \\
\text { Order }\end{array}$ & $\begin{array}{l}\text { Linear } \\
\text { Eighth- } \\
\text { Order }\end{array}$ & $\begin{array}{l}\text { Nonlinear } \\
\text { Second- } \\
\text { Order }\end{array}$ & $\begin{array}{l}\text { Nonlinear } \\
\text { Eighth- } \\
\text { Order }\end{array}$ \\
\hline 740-m Identification & .048 & .061 & .031 & .017 \\
\hline 740-m Verification & .051 & .049 & .036 & .026 \\
\hline 1200-m Identification & .051 & .059 & .032 & .011 \\
\hline 1200-m Verification & .070 & .058 & .064 & .026 \\
\hline 2500-m Identification & .030 & .034 & .027 & .006 \\
\hline 2500-m Verification & .047 & .051 & .045 & .014 \\
\hline
\end{tabular}

Table 6-I: RMS Velocity Error, m/s

\subsection{Massless Models}

One is inclined to suspect that inertial elements in the above models might be negligible, given the extremely small accelerations and apparent dominance of drag and tension terms. Such a shortened approach yields systems with half the states of the inertial models; the immediate numerical benefits are significant. This possibility will be briefly examined here, for the cases of single-ordered and fourth-ordered nonlinear models, applied to the 1200-meter identification and verification (AUTEC) runs.

The first-order equation is simply written as a contraction of (6.2): 


\begin{tabular}{|c|c|c|c|c|}
\hline & $\begin{array}{l}\text { Linear } \\
\text { Second- } \\
\text { Order }\end{array}$ & $\begin{array}{l}\text { Linear } \\
\text { Eighth- } \\
\text { Order }\end{array}$ & $\begin{array}{l}\text { Nonlinear } \\
\text { Second- } \\
\text { Order }\end{array}$ & $\begin{array}{l}\text { Nonlinear } \\
\text { Eighth- } \\
\text { Order }\end{array}$ \\
\hline 740-m Identification & 11.5 & 9.1 & 5.6 & 4.0 \\
\hline 740-m Verification & 20.2 & 20.6 & 8.8 & 6.7 \\
\hline 1200-m Identification & 9.6 & 7.8 & 4.5 & 1.6 \\
\hline 1200-m Verification & 13.3 & 13.6 & 8.6 & 4.6 \\
\hline 2500-m Identification & 14.6 & 10.3 & 11.0 & 3.7 \\
\hline 2500-m Verification & 2.4 & 3.9 & 2.2 & 1.5 \\
\hline
\end{tabular}

Table 6-II: RMS Position Error, $\mathrm{m} / \mathrm{s}$

$$
0=-\alpha\left(x_{v e h}-x_{\text {ship }}(t-\tau)-\beta \frac{d x_{v e h}}{d t}\left|\frac{d x_{v e h}}{d t}\right|\right.
$$

or, combining the unknowns into one:

$$
\frac{d x_{v e h}}{d t}=\sqrt{\left|\gamma\left(x_{v e h}-x_{\text {ship }}(t-\tau)\right)\right|} \operatorname{sgn}\left(-\gamma\left(x_{\text {veh }}-x_{\text {ship }}(t-\tau)\right)\right)
$$

This form now only has one parameter, and the time delay can still be included explicitly. 
A higher-order link model can be found from bond graphing techniques to be:

$$
\left[\begin{array}{c}
e_{1}^{\prime} \\
e_{2}^{\prime} \\
\cdot \\
e_{n-1} \\
e_{n}{ }^{\prime}
\end{array}\right]=\left[\begin{array}{cccc}
\frac{-k_{1}}{b} & \frac{k_{1}}{b} & & 0 \\
\frac{k_{2}}{b} & \frac{-2 k_{2}}{b} & \frac{k_{2}}{b} & \\
\cdot & \cdot & \cdot \\
& \frac{k_{n-1}}{b} \frac{-2 k_{n-1}}{b} & \frac{k_{n-1}}{b} \\
0 & & \frac{k_{n}}{b} & \frac{-k_{n}}{b}-\frac{k_{n}}{B_{v e h}}
\end{array}\right]\left[\begin{array}{l}
e_{1} \\
e_{2} \\
\cdot \\
e_{n-1} \\
e_{n}
\end{array}\right]+\left[\begin{array}{l}
k_{1} \\
0 \\
\cdot \\
0 \\
0
\end{array}\right] V_{\text {ship }}
$$

where $b=\gamma_{n}\left|f_{i}\right|$ if $\gamma_{n}$ is the quadratic drag multiplier. This model requires only two parameters, the drag characteristics of the cable and of the vehicle.

Figures 6-11 and 6-12 show the error associated with the massless models compared to that of the second- and eighth-order forms of the previous section, for the 1200-meter identification run. This comparison is made again for the 1200-meter experimental run in Figures 6-13 and 6-14. In both cases, the inertia-free model is seen to give results extremely similar to those of the more complex case. The rms-velocity error for identification is .0336 meters/second for the first-order model, and .01039 meters/second for the fourth-order form. Similarly, the rms-velocity error for the experiment is .0501 meters/second for the first-order model, and .0263 meters/second for the eighth-order form. Comparison of these results with Table 6-I is quite favorable, and position error comparisons follow the same trends.

The good performance of the reduced-order models is fortunate, for significant identification time reductions are realized, due to several reasons. First, not only are half of the states nonexistent, but in addition the stiff modes have slowed down or disappeared, allowing the integrator to take larger steps with confidence. The 
computation speed improvement due to dropping the inertial terms has typically been at least an order of magnitude for the same input stream. Thus, each "learning step" simulation has been significantly shortened. Further, with the reduction of the parameter vector to two elements instead of four (in the four-link case), it also takes far fewer steps during the parameter search to find the minimum error. Thus, for these reasons, as well as the more esoteric implications of an excessively large parameter vector, the reduced forms are far preferred for their performance.

\subsection{Relationship of Link Model Parameters to Real Quantities}

As stated before, in a model which discretizes the cable into segments, initial parameter guesses can be made by sectioning the continuous quantities of the cable, and the goodness of those first estimates should improve for higher resolution. For the four-link system we have been working with, it is interesting to note the correlation between first guesses obtained in this manner, and the final parameters found by the identification algorithm. Table 6-III presents this data, for all three of the identified runs at 740, 1200, and 2500 meters. The mapped continuous quantities are based on a simple lumping of the cable drag and mass properties along the cable length.

Discrepancies between continuous quantities and values found through identification are not to be regarded as troublesome errors in any real sense, since the continuous and discrete-length systems are physically very different, and the performance of the models has been shown to be good. However, some correlations can be noted, providing insight into the identification results. If we can take the continuous quantity mapped values as "truth", the most interesting trend of the table is that the eighth-order models have greatly reduced the importance of vehicle drag and inertia, and, in fact, placed some of these forces onto the cable's upper links through increased cable drag and effective mass coefficients. On the contrary, the inertia-free models possess consistently low cable drag 
$-79-$
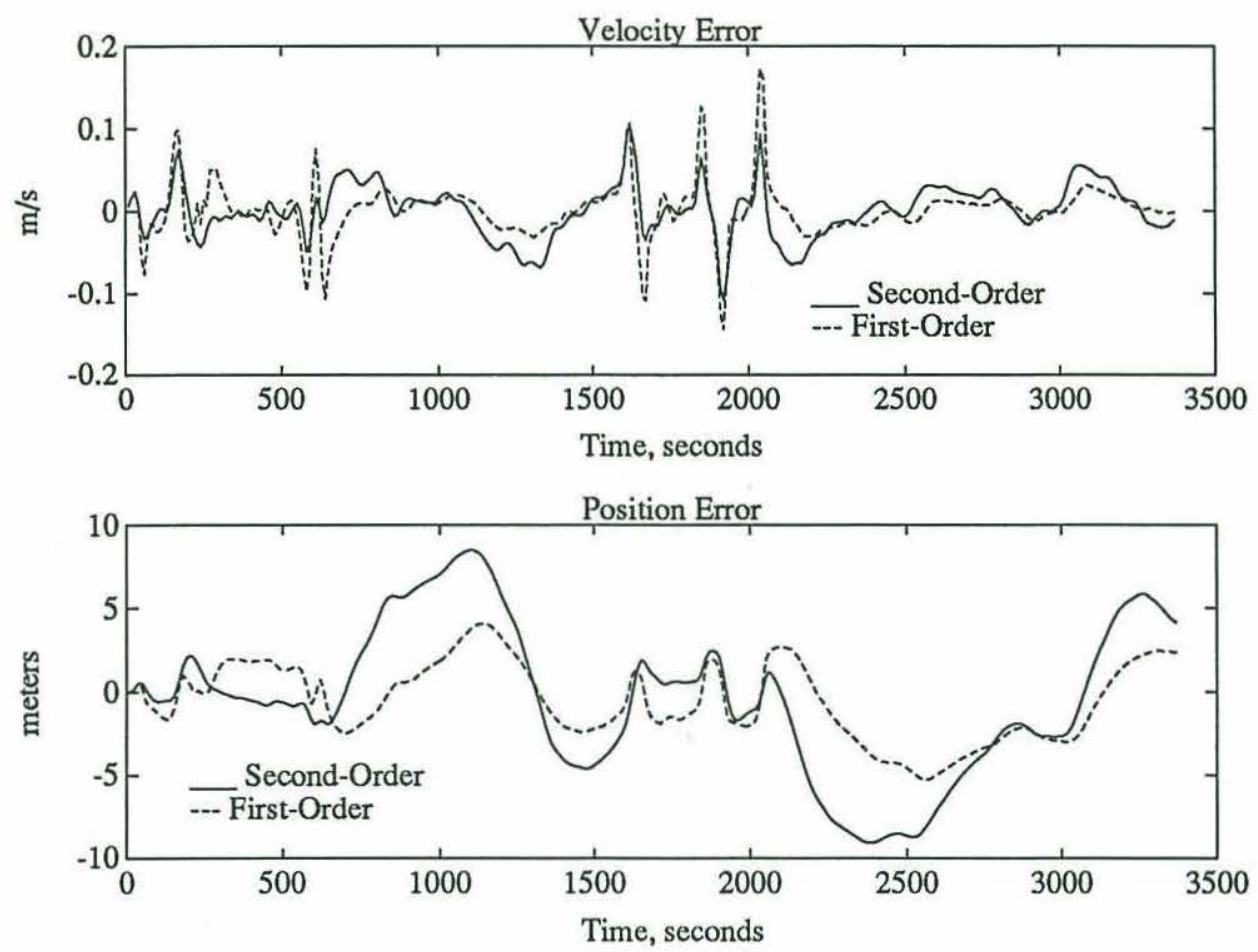

Figure 6-11: First- vs. Second-Order Models: Identification Run
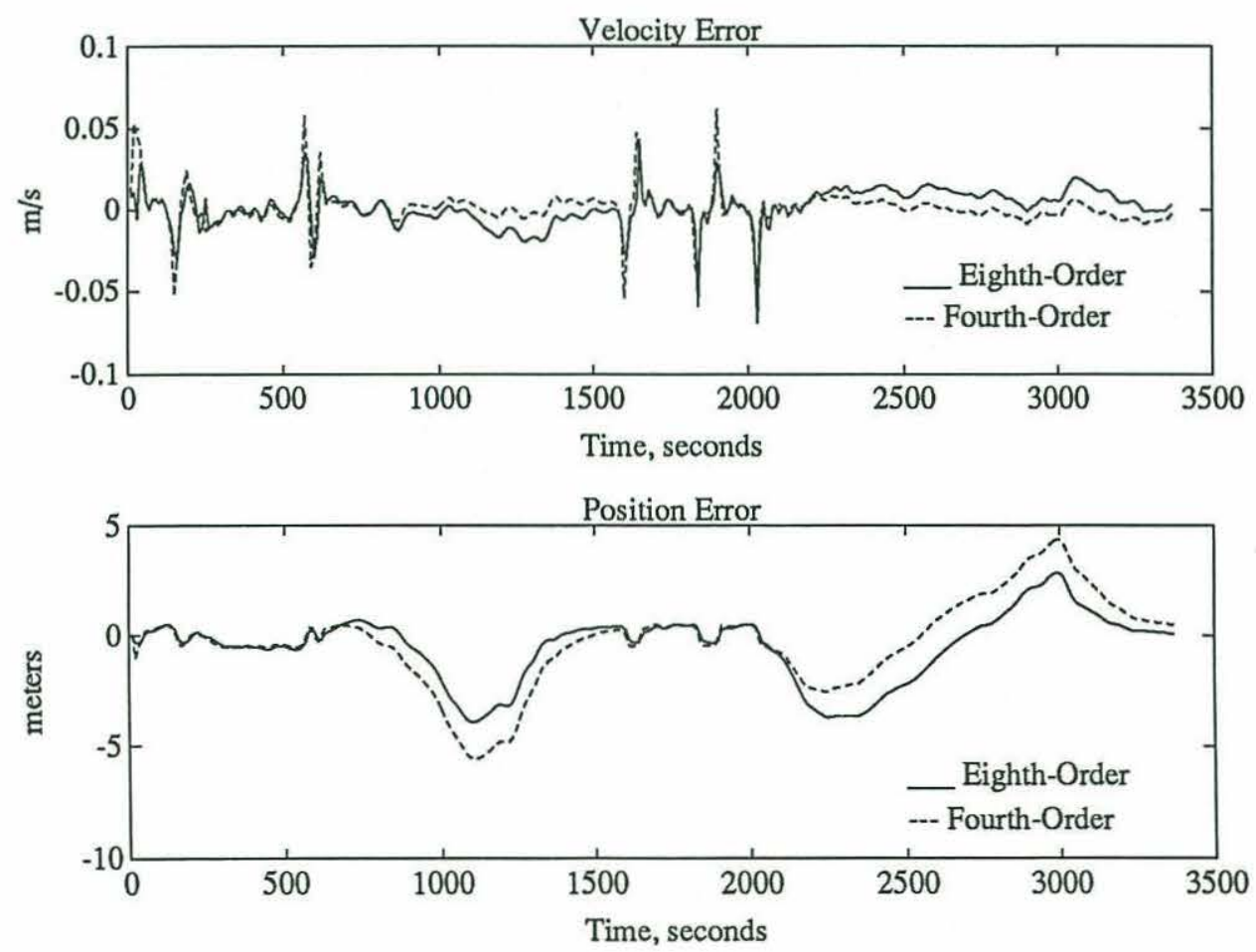

Figure 6-12: Fourth- vs. Eighth-Order Models: Identification Run 
$-80-$
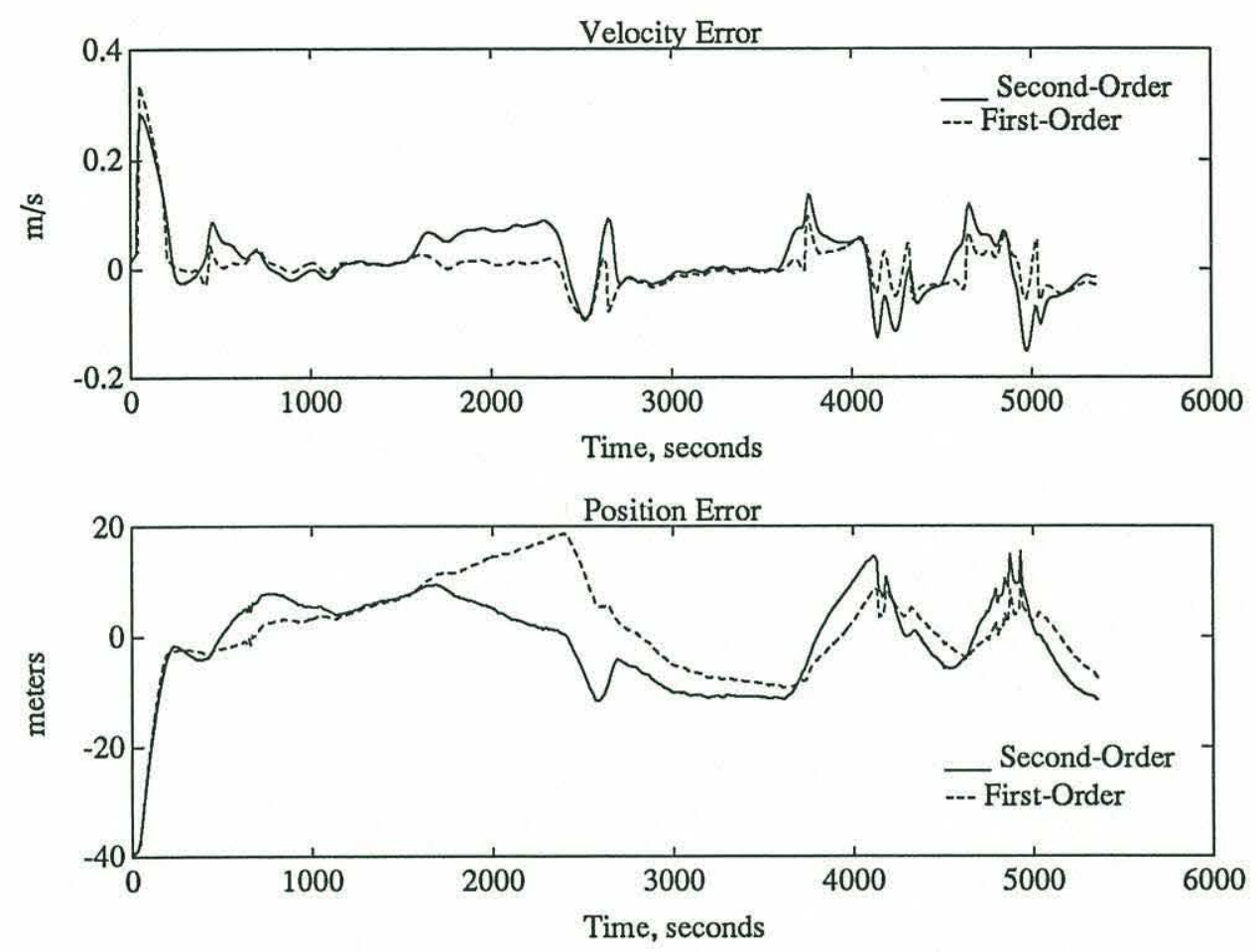

Figure 6-13: First- vs. Second-Order Models: Experimental Run
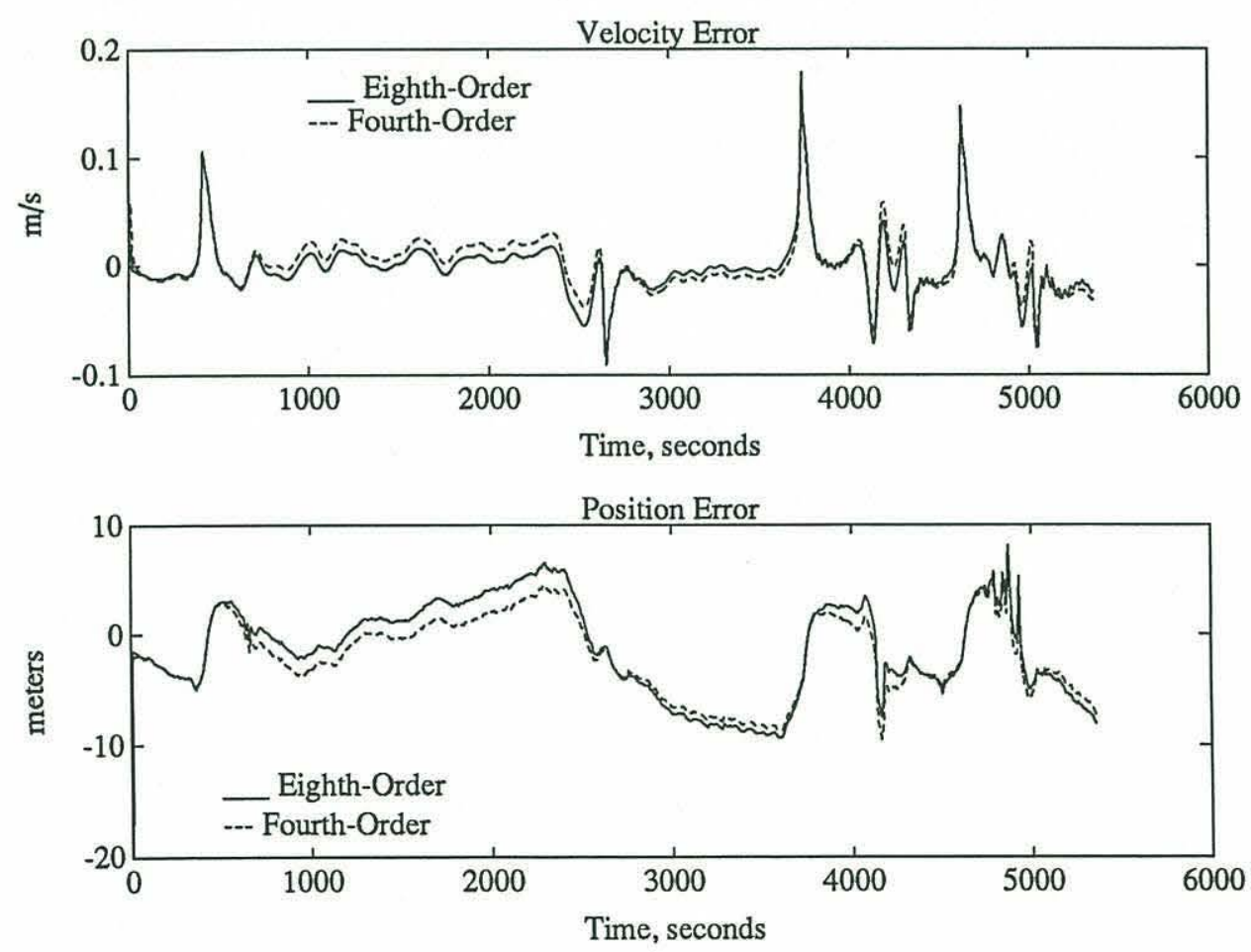

Figure 6-14: Fourth- vs. Eighth-Order Models: Experimental Run 


\begin{tabular}{|c|c|c|c|c|}
\hline & & $\begin{array}{l}\text { Continuous- } \\
\text { Quantity } \\
\text { Mapping }\end{array}$ & $\begin{array}{l}\text { Nonlinear } \\
\text { Elghth- } \\
\text { Order }\end{array}$ & $\begin{array}{l}\text { Nonlinear } \\
\text { Fourth- } \\
\text { Order }\end{array}$ \\
\hline \multirow{4}{*}{$740 \mathrm{~m}$} & Cable $C_{D},\left(\frac{N}{m}\right)\left(\frac{s^{2}}{m^{2}}\right)$ & 13.84 & 24.80 & 10.73 \\
\hline & Cable Eff. Mass, $\frac{\mathrm{kg}}{\mathrm{m}}$ & 1.275 & 3.742 & - \\
\hline & Vehicle $C_{D},(N)\left(\frac{s^{2}}{m^{2}}\right)$ & 416 & .0021 & 3803 \\
\hline & Vehicle Eff. Mass, kg & 1305 & 171 & 一 \\
\hline \multirow{4}{*}{$1200 \mathrm{~m}$} & Cable $C_{D},\left(\frac{N}{m}\right)\left(\frac{s^{2}}{m^{2}}\right)$ & 13.84 & 18.57 & 13.71 \\
\hline & Cable Eff. Mass, $\frac{\mathrm{kg}}{\mathrm{m}}$ & 1.275 & 3.138 & - \\
\hline & Vehicle $C_{D},(N)\left(\frac{\mathrm{s}^{2}}{\mathrm{~m}^{2}}\right)$ & 110 & .0018 & 4469 \\
\hline & Vehicle Eff. Mass, kg & 2285 & 101 & - \\
\hline \multirow{4}{*}{$2500 \mathrm{~m}$} & Cable $C_{D},\left(\frac{N}{m}\right)\left(\frac{s^{2}}{m^{2}}\right)$ & 13.84 & 27.31 & 13.02 \\
\hline & Cable Eff. Mass, $\frac{\mathrm{kg}}{\mathrm{m}}$ & 1.275 & 4.609 & - \\
\hline & Vehicle $C_{D},(N)\left(\frac{\mathrm{s}^{2}}{\mathrm{~m}^{2}}\right)$ & 416 & .0023 & 9887 \\
\hline & Vehicle Eff. Mass, kg & 1305 & 146 & 一 \\
\hline
\end{tabular}

Table 6-III: Comparison of Initial Parameter Estimates to Identified Parameters

terms, apparently putting more stress on drag at the vehicle. A short sensitivity analysis has shown the performance of the eighth-order model is quite robust to changes in vehicle properties, and extremely vulnerable to changes in cable drag and mass 
coefficients. For the fourth-order form, the effects of parameter tuning on the cable and vehicle are of the same order.

The best correlation between a continuous quantity and its counterpart from identification clearly is for the cable drag coefficient of the cable. In all three cases, the continuous value of $13.48 \frac{N-\sec ^{2}}{\mathrm{~m}^{3}}$ is reasonably maintained within about $23 \%$ for the inertia-free models, and within a factor of two for the eighth-order model with masses. By attempting to "track" some of the lost and gained vehicle drag effect onto the cable, we can account for varying parts of the discrepancy; most of them are not significant, however. 


\begin{tabular}{|c|c|c|}
\hline \multicolumn{3}{|c|}{ Lost Vehicle } \\
\hline & Drag Effect & Cable Drag \\
\hline & to Cable & Discrepancy \\
\hline $740 \mathrm{~m}$ - 8th-order & +.560 & +10.96 \\
\hline $1200 \mathrm{~m}-8$ th-order & +.092 & +4.73 \\
\hline $2500 m$ - 8th-order & +.166 & +13.47 \\
\hline $740 m$ - 4th-order & -4.57 & $-3.11 *$ \\
\hline $1200 \mathrm{~m}$ - 4th-order & -3.63 & -.13 \\
\hline $2500 \mathrm{~m}-4$ th-order & -3.78 & -.82 \\
\hline
\end{tabular}

A similar effort to trace the inertial effects for the eighth-order from the vehicle to the cable gives slightly more encouraging results:

\section{Lost Vehicle \\ Inertial Effect \\ $\underline{\text { to Cable Mass }}$ \\ Cable Mass \\ $\underline{\text { Discrepancy }}$}

$740 \mathrm{~m}$

$1200 \mathrm{~m}$

$2500 \mathrm{~m}$
$+1.53$

$+1.82$

$+.46$
$+2.46$

+1.86 *

$+3.34$ 
Suprisingly, a similar calculation for the nonlinear second-order models shows that the relation between initial guesses and the final identified values is by no means worse than in the higher-ordered case:

$\begin{array}{lcc} & \begin{array}{c}\text { Predicted } \\ \mathrm{b} / \mathrm{k}\end{array} & \begin{array}{c}\text { Identified } \\ \mathrm{b} / \mathrm{k}\end{array} \\ 740 \mathrm{~m} & 459 & 571 \\ 1200 \mathrm{~m} & 425 & 500 \\ 2500 \mathrm{~m} & 2778 & 4833\end{array}$

Here, the spring constant is based on midpoint tension, and the drag is taken to include the vehicle plus the lower half of the cable. Mass terms are neglected since they have been found unimportant in an earlier section.

This unexpected comparison of low- and high- order parameter correlations to physical quantities is contrary to our previous intuitions concerning the physical relevance of final identified paramet; we had expected that the parameters would become more meaningful for increasing resolution. In addition, we are still at a loss to explain why the fourth- and eighth-order model identifications perform unique allocations of mass and drag between the links and the vehicle.

Clearly, the constraint to two and four parameters for the four-link models still allows for some nonuniqueness: that is, we have no guarantee that the identified parameters found in Table 6-III are the right values, or the only values, for which the model response is accurate. The evolution of a single identified parameter, $b / k$, for the one-link case, is more comprehensible. Nonetheless, given that the objective is to maximize performance of the models, the inertia-free nonlinear four-link model is the best among all those 
$-85-$

considered in this work. An efficient and sensible identification result is the penalty in this case, but it can be argued that better model structures might be able to identify the systems from a more physically-motivated viewpoint.

\subsection{Appendix}

The following pages contain the error time series from which Tables 6-I and 6-II were created. They clearly show the superiority of the nonlinear forms and, on occasion, the advantages of the higher-order models. 
$-86-$
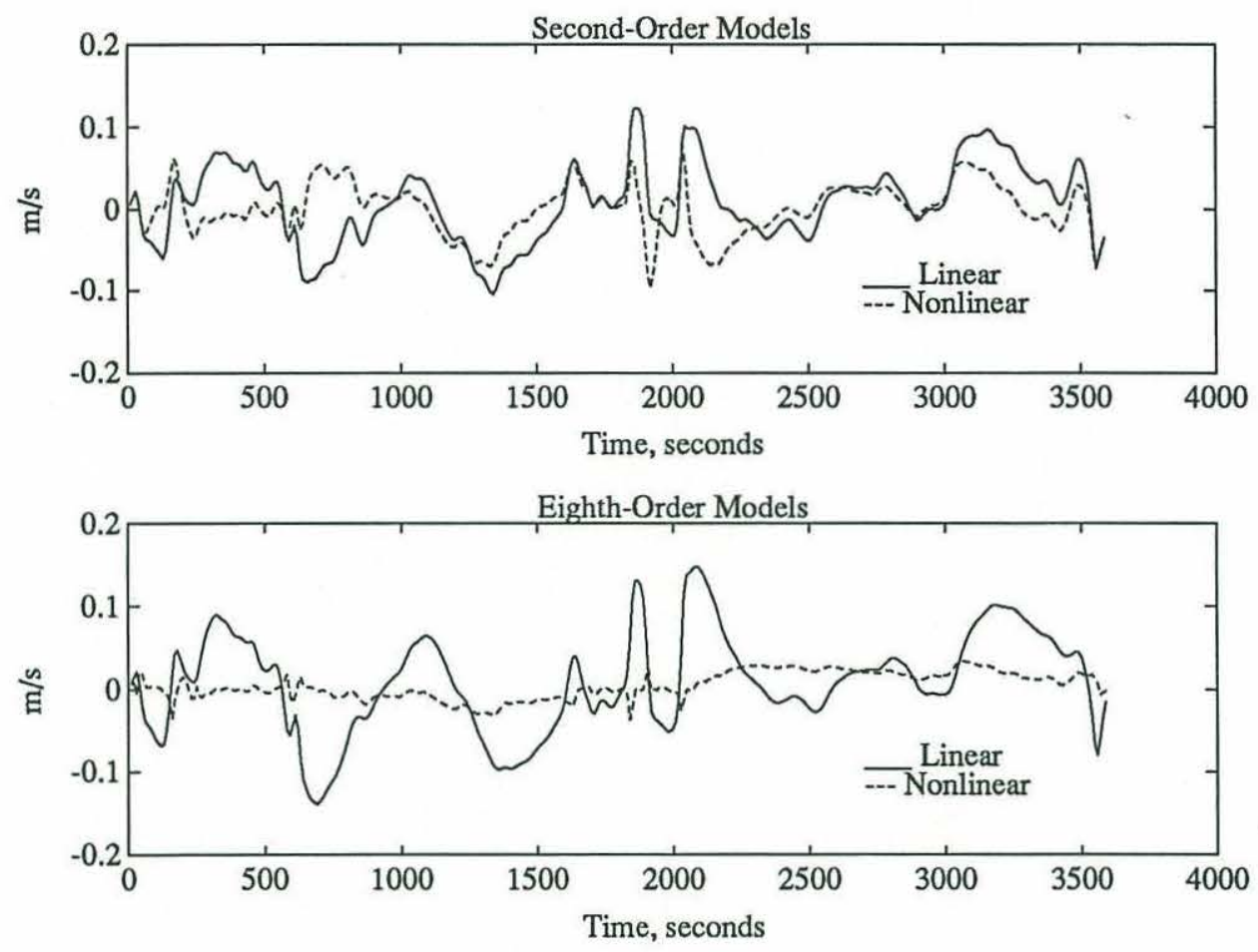

Figure 6-15: Identification Run for 740 Meters: Velocity Error
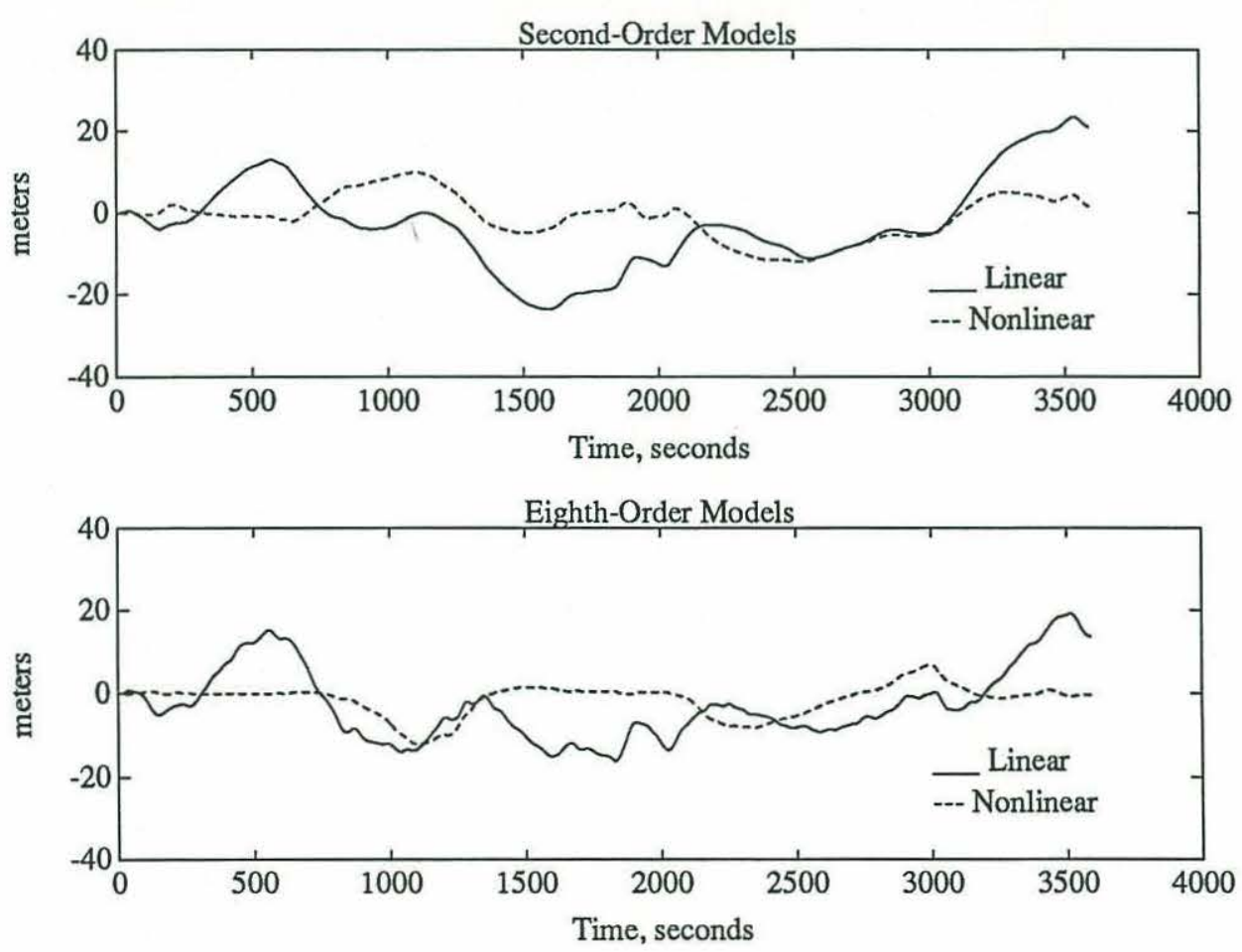

Figure 6-16: Identification Run for 740 Meters: Position Error 
$-87-$
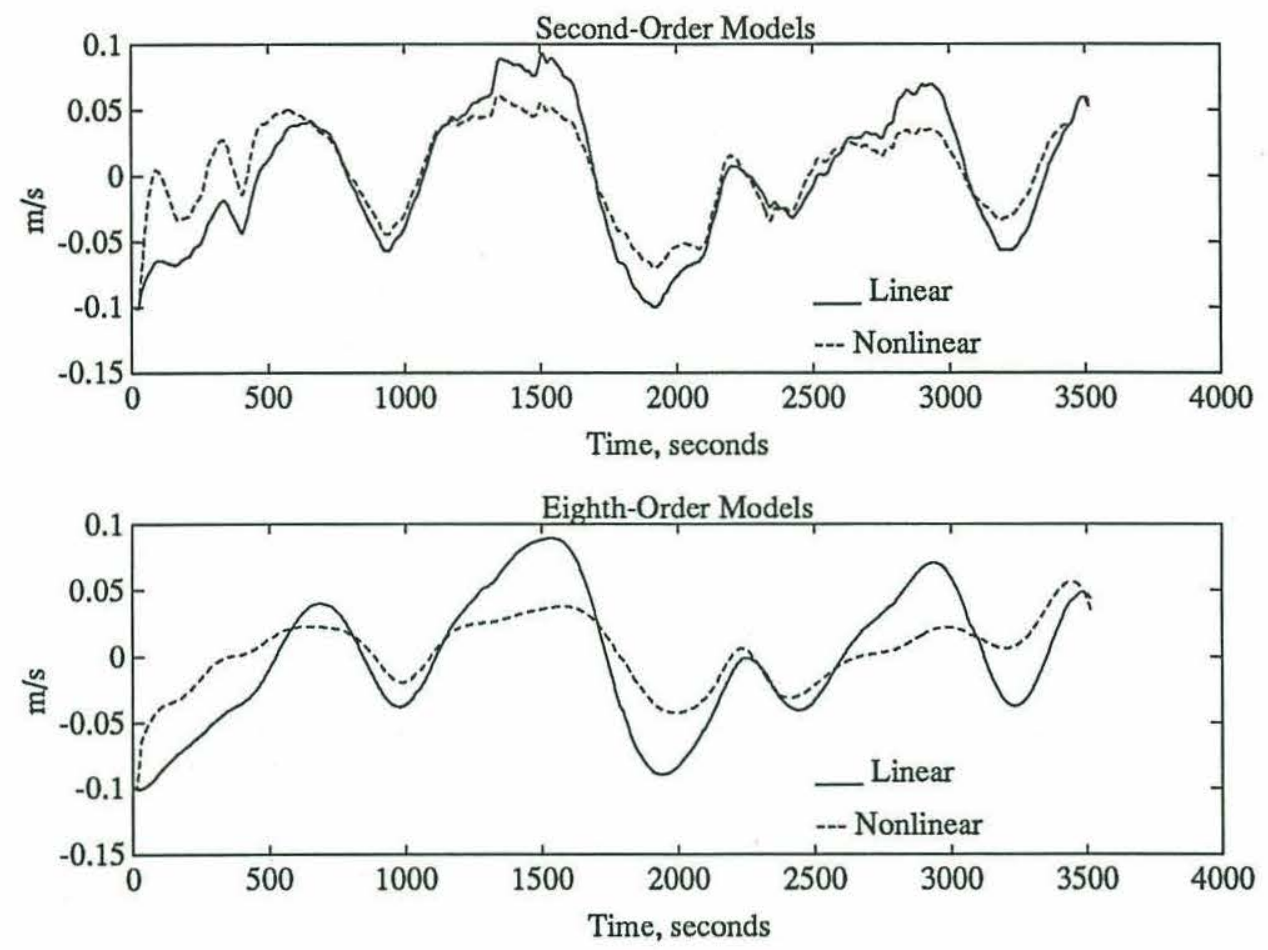

Figure 6-17: Verification Run for 740 Meters: Velocity Error
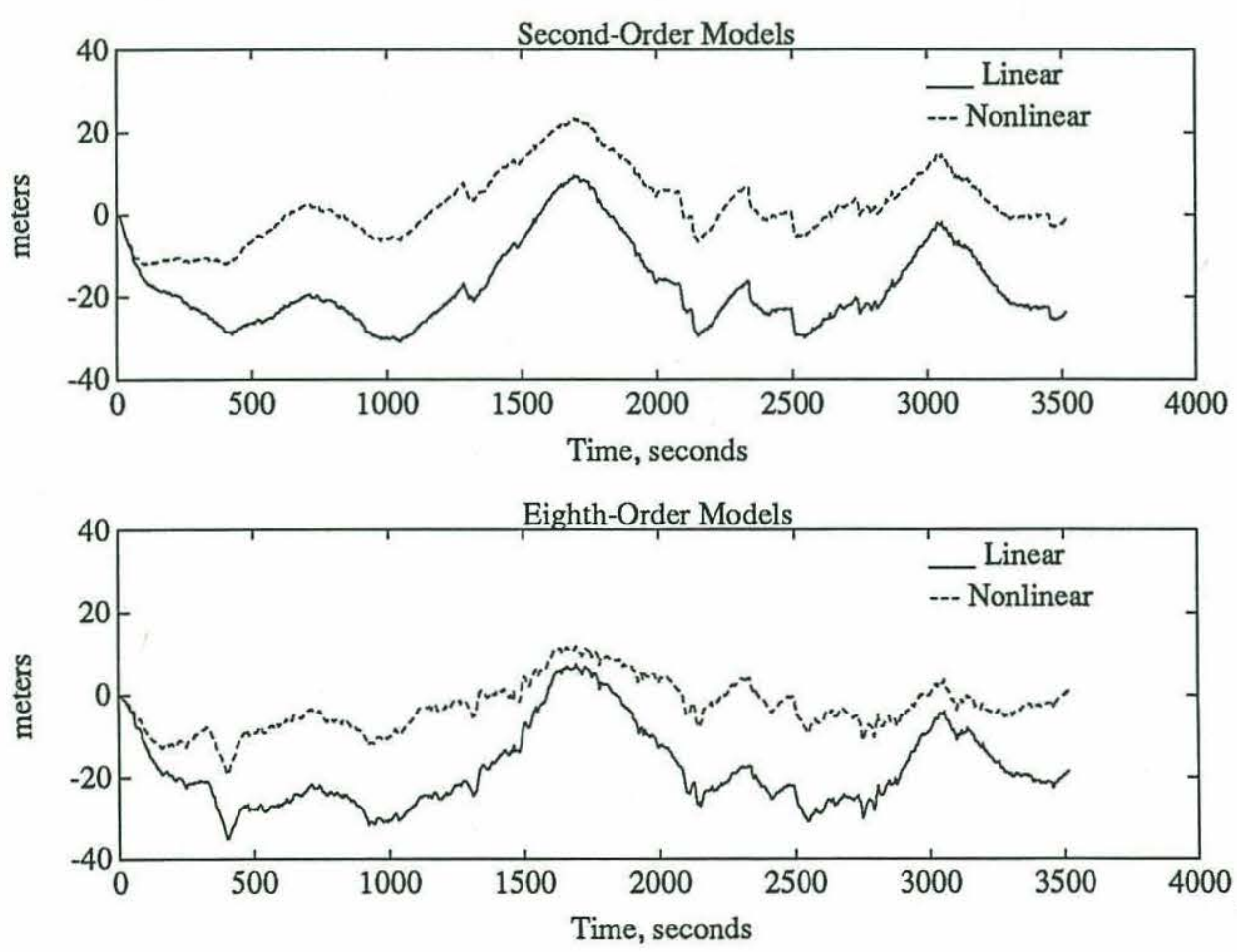

Figure 6-18: Verification Run for 740 Meters: Position Error 
$-88-$
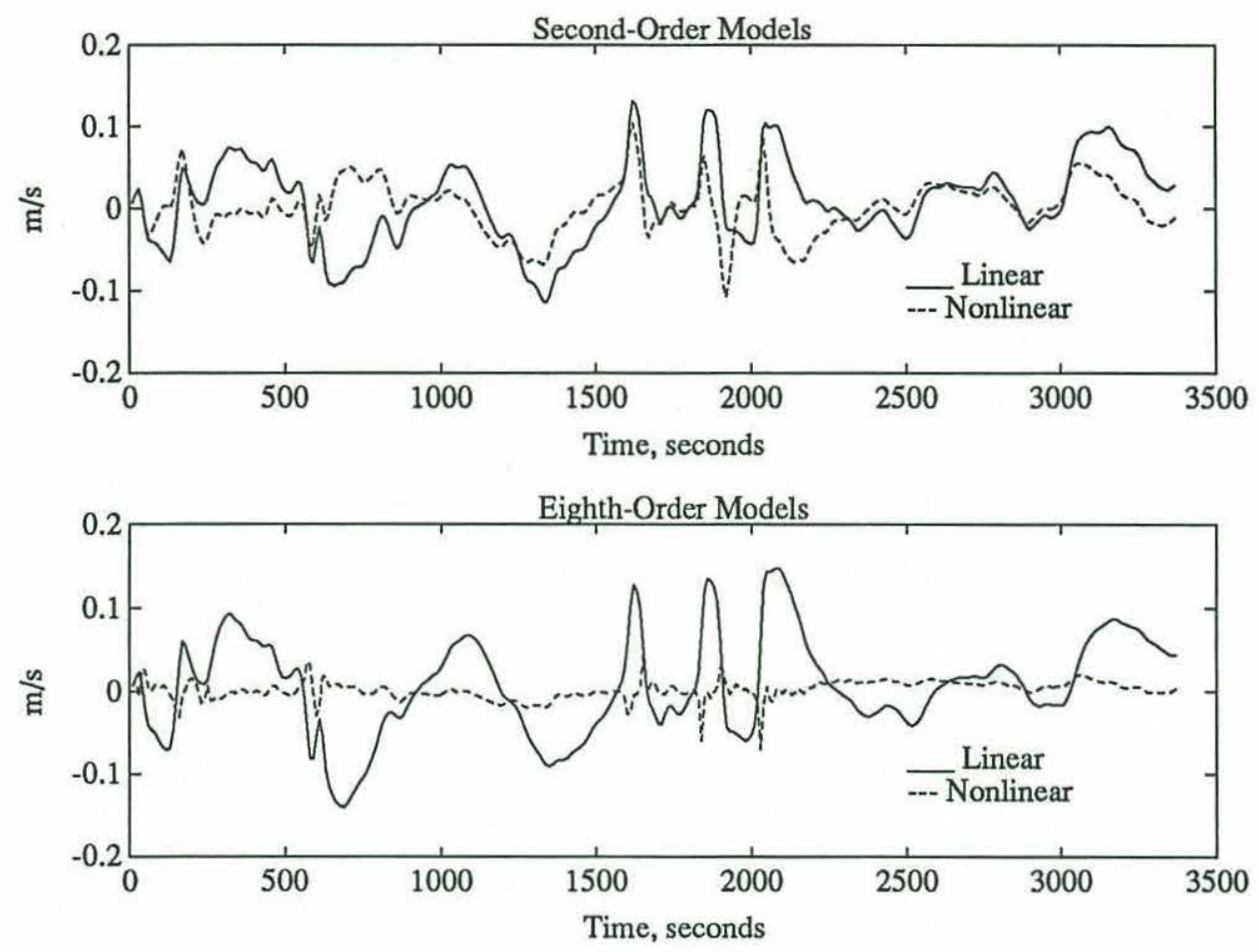

Figure 6-19: Identification Run for 1200 Meters: Velocity Error
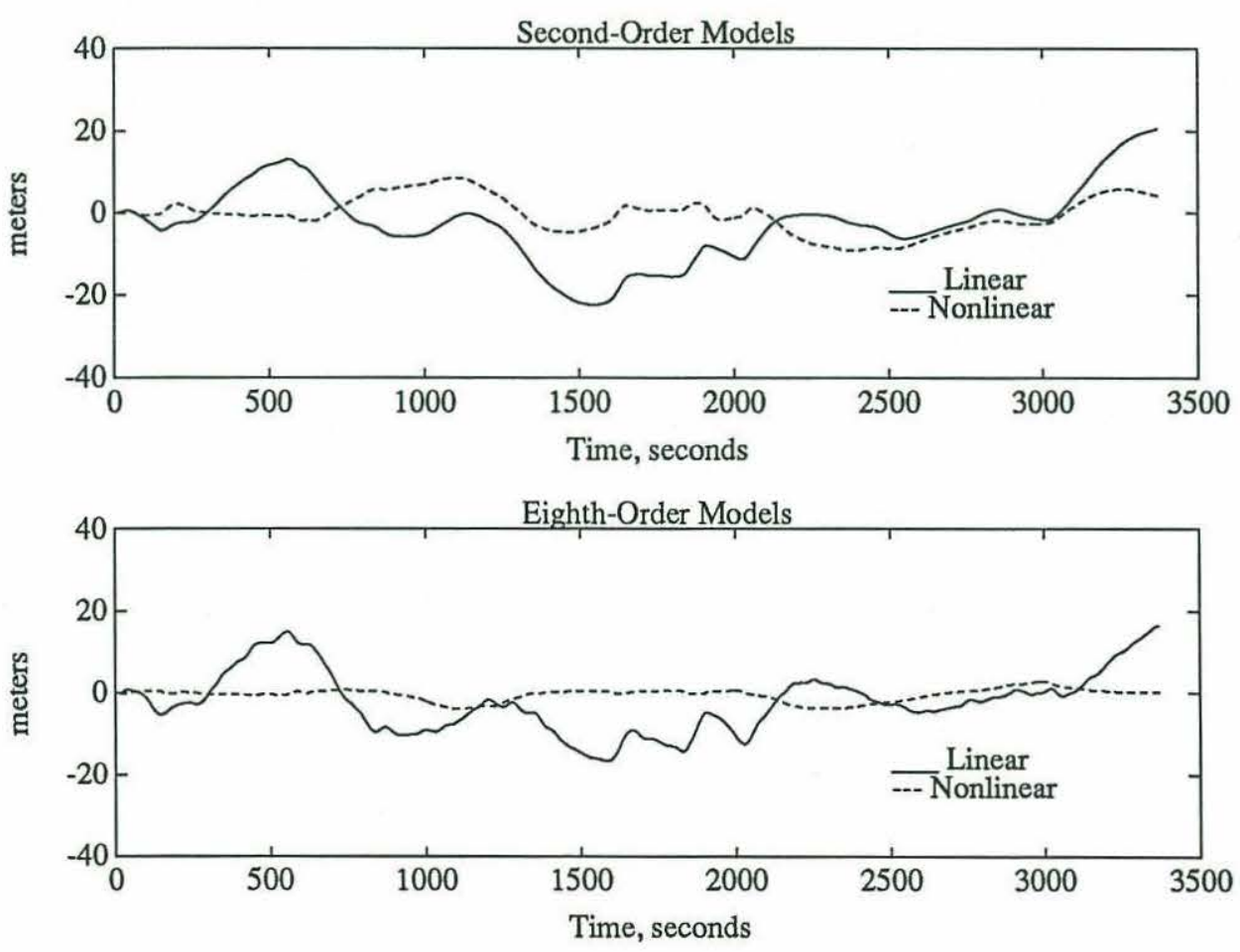

Figure 6-20: Identification Run for 1200 Meters: Position Error 
$-89-$
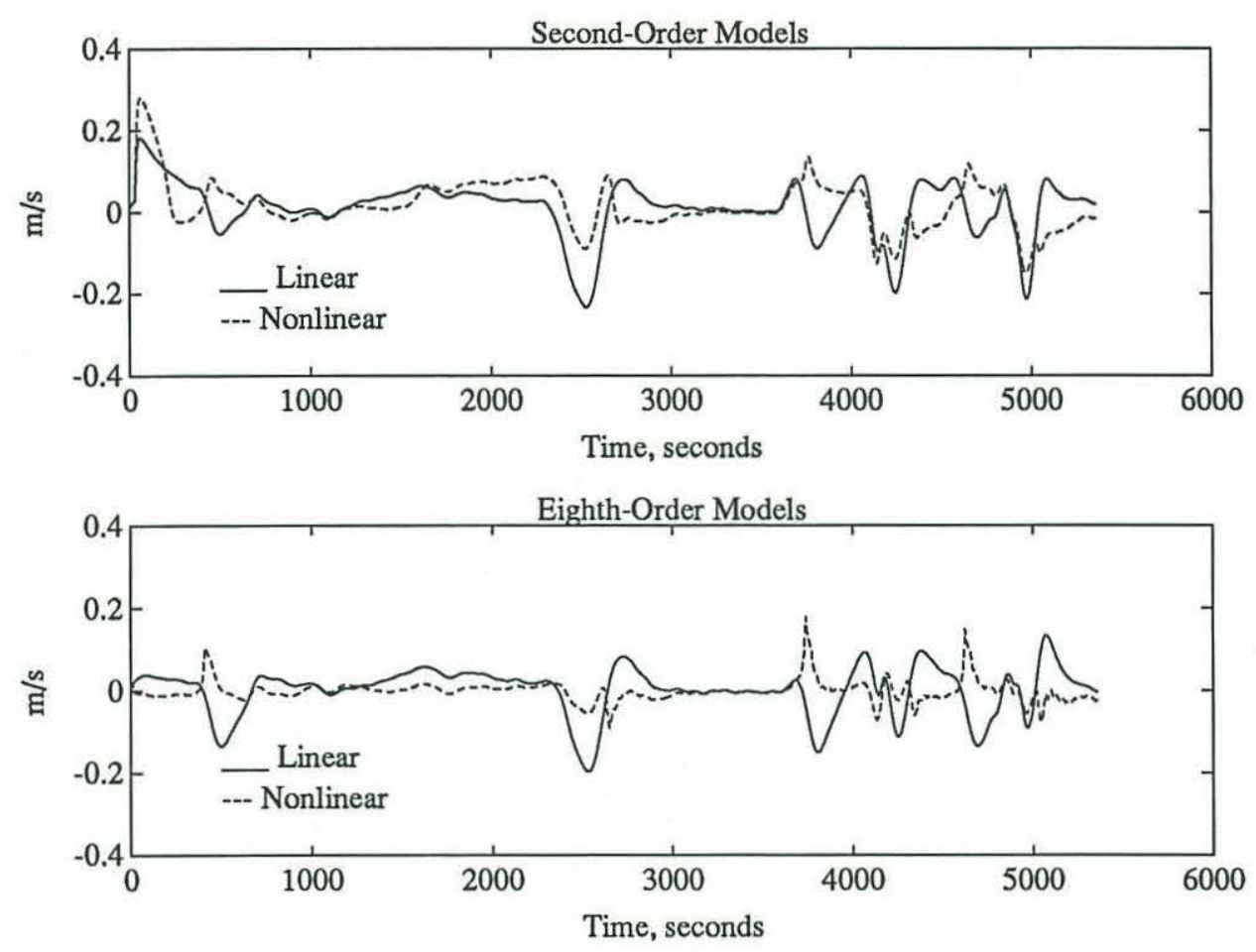

Figure 6-21: Verification Run for 1200 Meters: Velocity Error
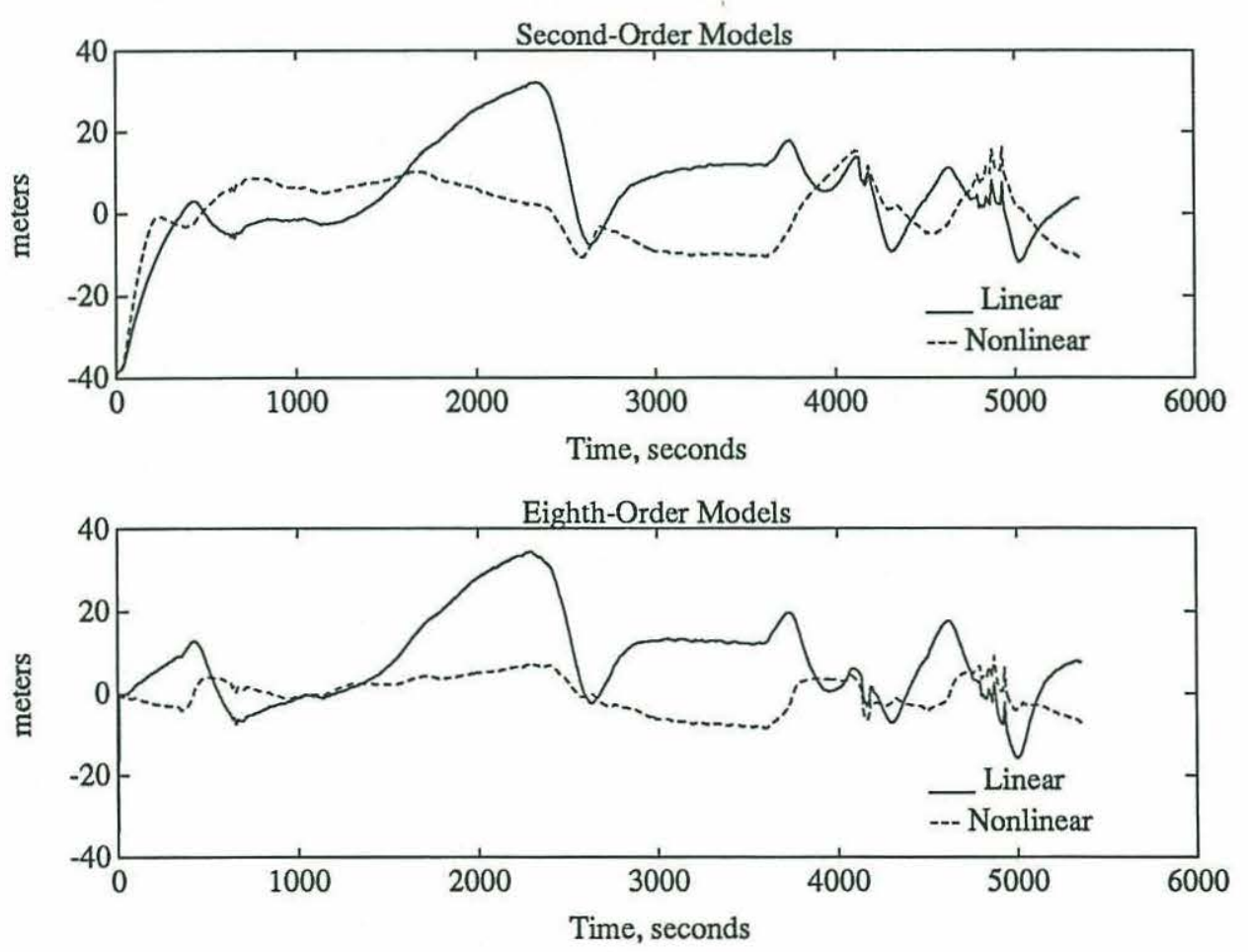

Figure 6-22: Verification Run for 1200 Meters: Position Error 

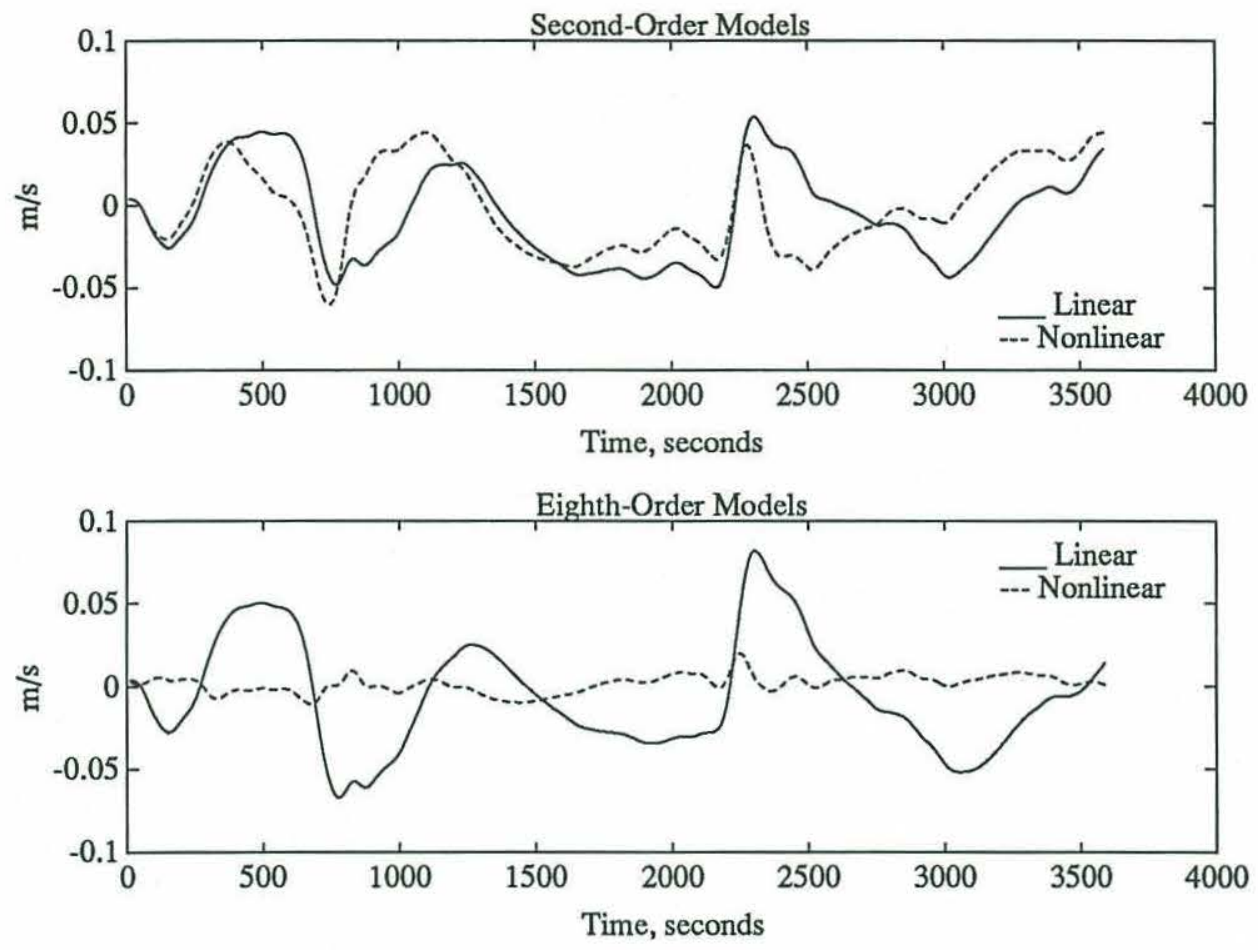

Figure 6-23: Identification Run for 2500 Meters: Velocity Error
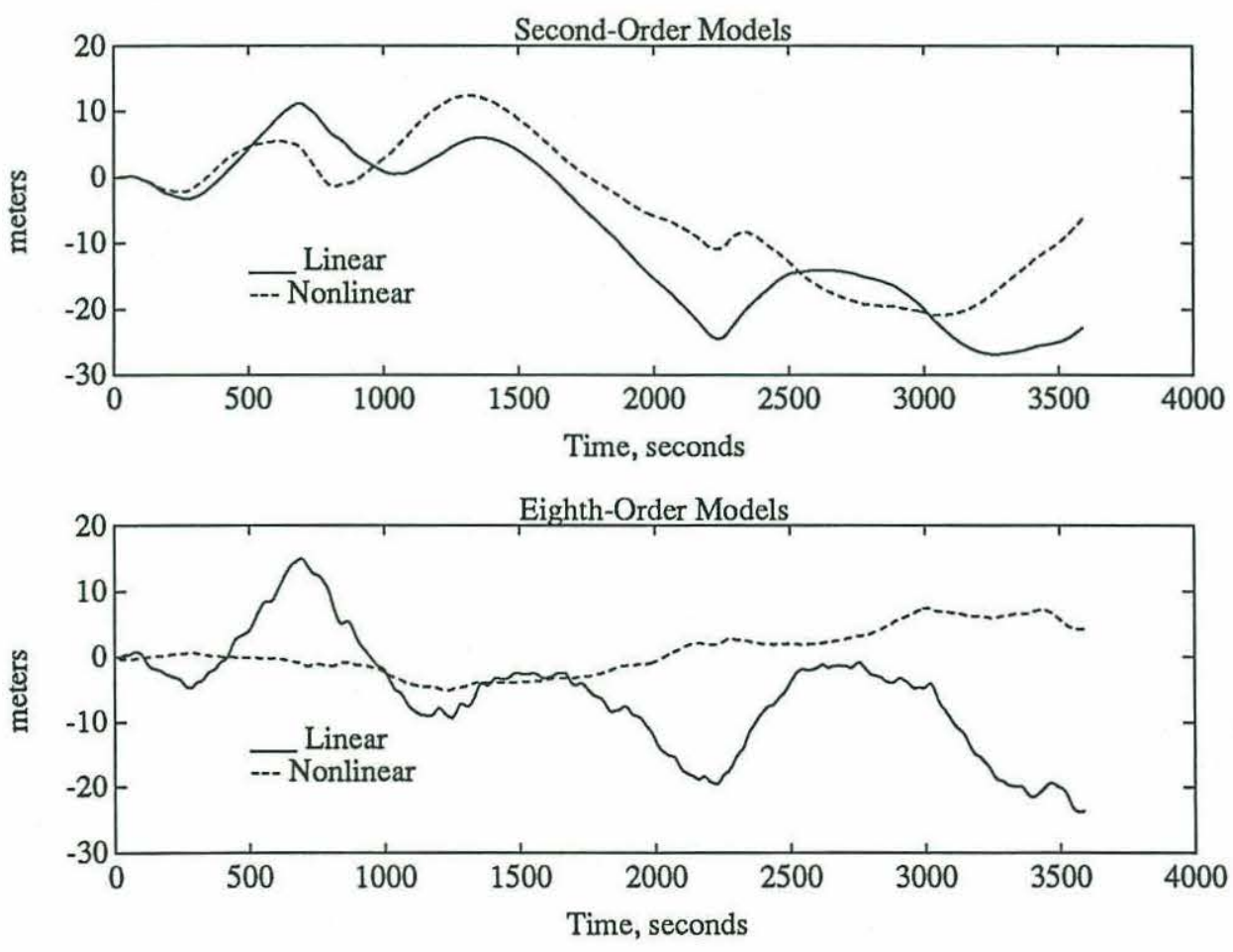

Figure 6-24: Identification Run for 2500 Meters: Position Error 
$-91-$
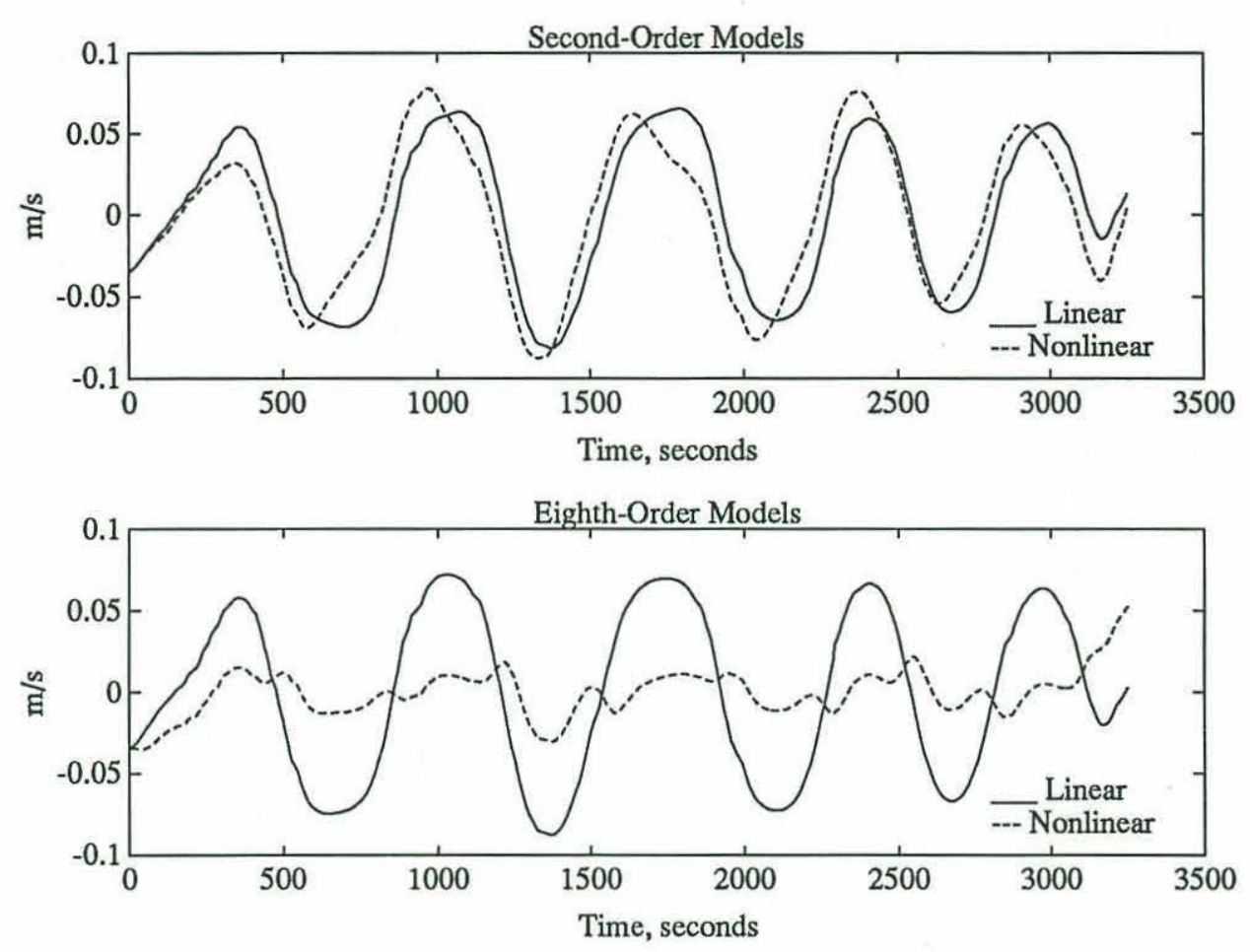

Figure 6-25: Verification Run for 2500 Meters: Velocity Error
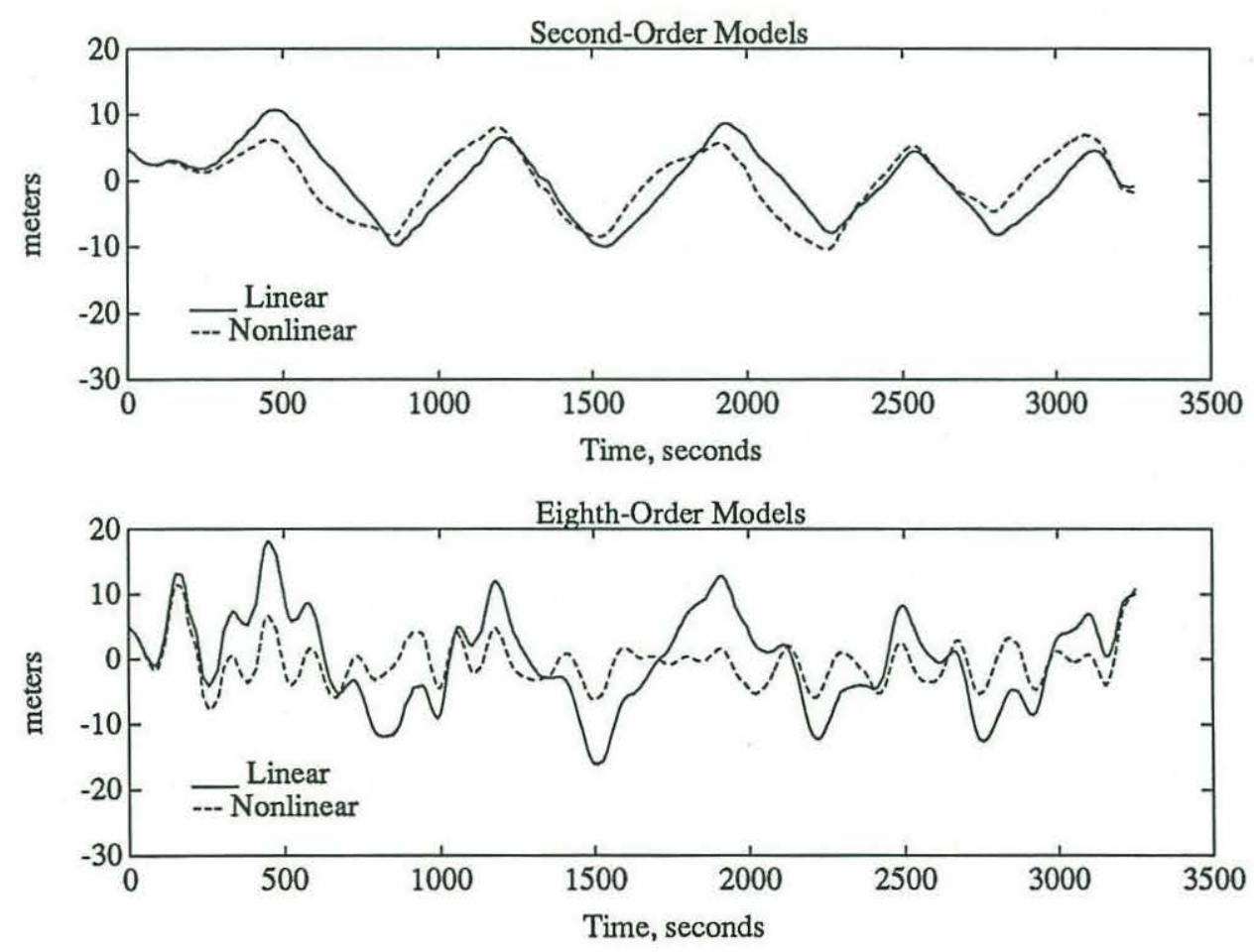

Figure 6-26: Verification Run for 2500 Meters: Position Error 


\section{Chapter 7}

\section{Conclusions and Recommendations}

In summary, the objective of this work was to develop a procedure for creating parameterized dynamic models of cable/vehicle systems like ARGO/JASON, that could be used in existing controller structures to improve the system performance. The first step was to gain some understanding of the real system; toward this end, an analytical model with known, physically-based parameters was described in Chapter 2, and qualitatively verified with actual sea data in Chapter 3. This verification took place in two regimes, one with rich frequency content and nonzero tow speeds, and another based solely on frequency-response measurements. In both instances, the analytical model provided excellent simulation results, with a minimum of parameter tuning.

With the insight of the analytical model, a very general discussion of several important system characteristics was given in Chapter 4; these included the pure time delay, and the formation and dissolution of the catenary. Further, an example showed how even a crude understanding of the system could be used to specify a practical and efficient ship trajectory for a point-to-point maneuver.

Chapter 5 presented the overall scheme for identification and verification; in Chapter 6 , the process was carried out for a number of parametric forms. The analytical model served as the basis for the identification loop, driven by a fictitious input shaped only by the bandwidth limits of the ship and a typical maximum velocity. Its output was taken as truth for the purposes of identification, providing a tool that could be used to characterize systems for which there was no real data available. This ability is seminal to the practical implementation of the results of this work: good models can be created a priori, for onshore controller design and immediate use at the site. 
Actual sea data was utilized concurrently to verify the parameterized models. Three distinct runs with unique dynamic characters formed the verification set, and all indications are that the best parametric models can respond to a variety of input scenarios consistently. Of the forms considered, higher-ordered multi-link nonlinear forms were shown to have the smallest simulation errors, and overall, neglecting inertial effects had little effect on the models' performances. Velocity plots for the verification runs are given in Figures 7-1, 7-2, and 7-3. Again, these curves represent responses from models created with the analytical model and a fictitious ship trajectory--no real data was used.

Finally, it was illustrated that the identification mechanism is not at all straightforward; the process in some instances gave inconsistent allocations of forces along the cable and at the vehicle, and there was evidence that the final solutions were not unique. Even in the worst cases of parameter deterioration, however, good performance was maintained. Although from these results the quality of the parameters appears to be fairly unimportant, further work on this issue is needed to establish true control over the identification process.

Implications of the error bounds specified in Chapter 6 can be of a general nature only, in light of the lack of experience in closed-loop vehicle trajectory control. Parameter uncertainty can be handled in many cases by robust or adaptive control methodologies (see, for example, [Slotine 89]). Given their suitability for this type of problem, these techniques will likely be pursued in the near future, and implemented in an outer control loop. From this robust perspective, the structure of the model and the identified parameters as a starting parameter vector for the adaptation are the important outcomes of the current work. In addition, the Smith Predictor [Ioannides 79] may take some significant role in improving control of the vehicle, by addressing explicitly the presence of a pure delay on the input. 


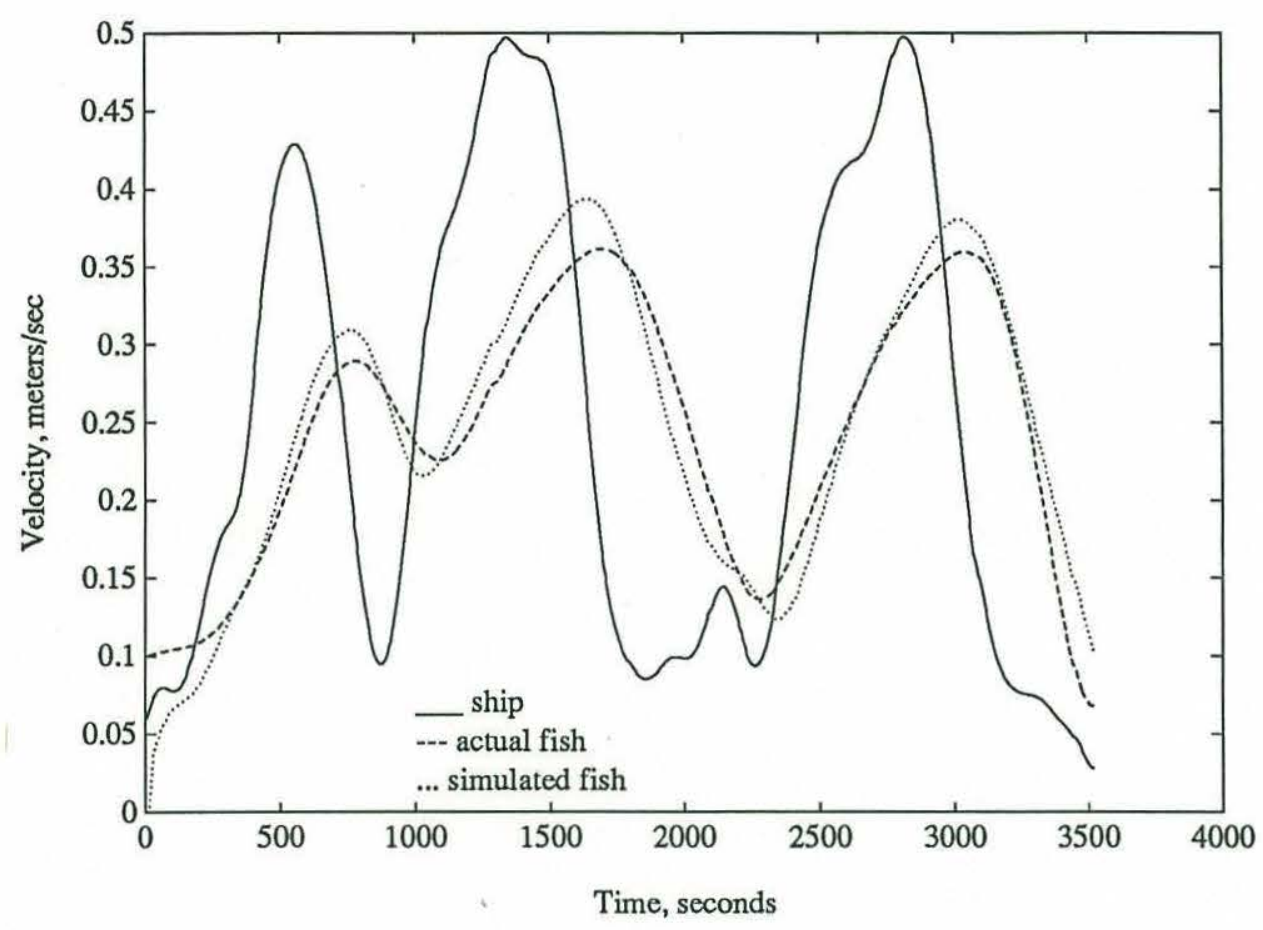

Figure 7-1: Actual and Simulated Fish Response--740 meters

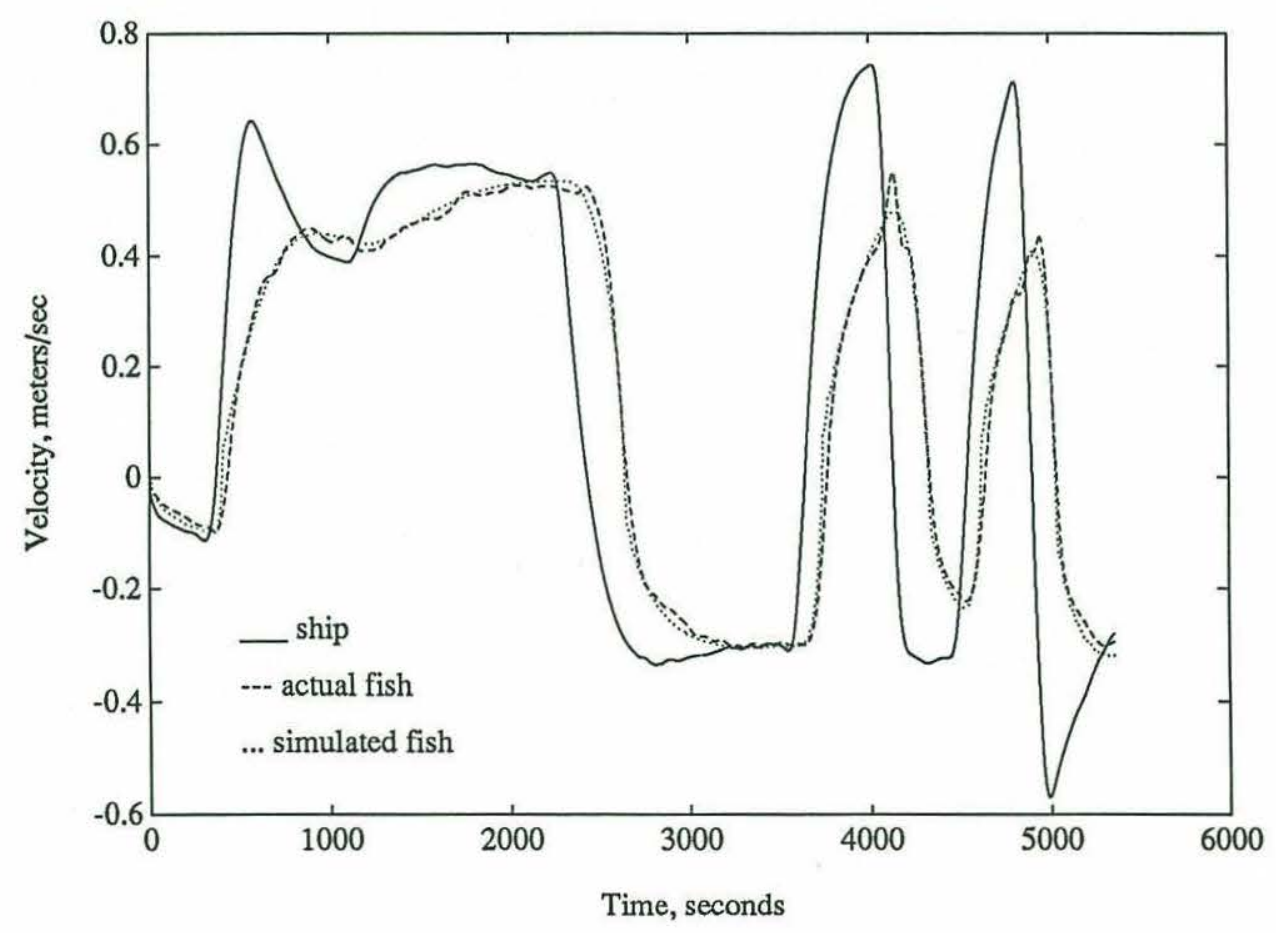

Figure 7-2: Actual and Simulated Fish Response--1200 meters 


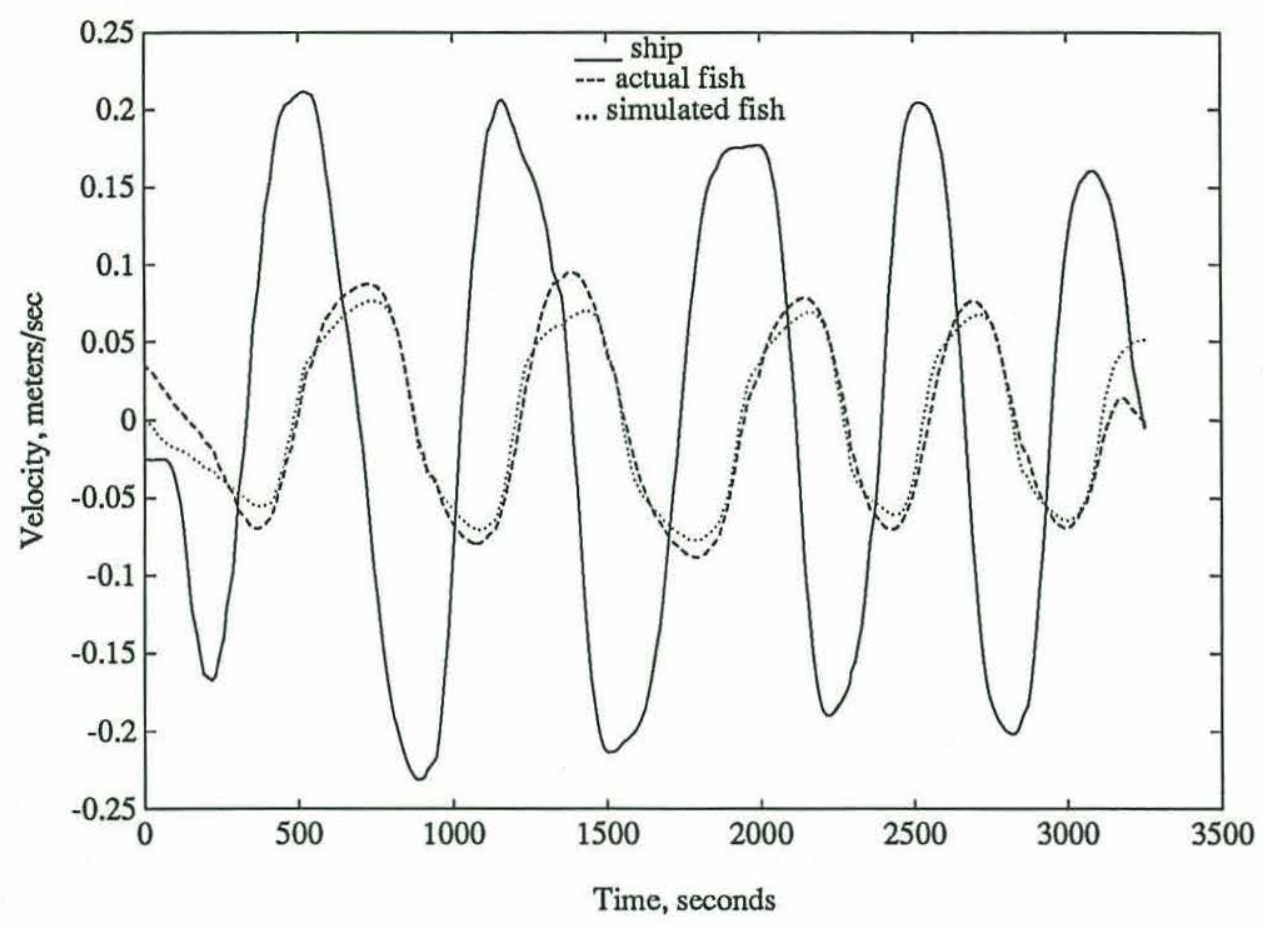

Figure 7-3: Actual and Simulated Fish Response--2500 meters

Other areas of future investigation should include further exploitation of the analytical model of Chapter 2; its capability for modelling heave and pitch motions of the vehicle, in the presence of significant lateral deflections of the cable, may transcend its treatment of horizontal motions. Investigation of snap-loading and otherwise high dynamic tensions can be addressed with this tool, and heave compensation schemes examined. A data set confirming the accuracy of the model with respect to these heave motions is essential to pursuing these issues. Also, extension of the modelling principles presented there to address out-of-plane motions is a straightforward task, one which has been accomplished in rough form, but has yet to be verified. Accurate modelling of the threedimensional responses can find a place in the control schemes as well, and will afford the operator an ability to cover a wider range of operational scenarios. Finally, the basic dynamic relations presented in Chapter 2 are applicable to a variety of cable problems, 
-96-

including taut cables which are not vertically oriented, and slack tethers between free bodies. 


\section{References}

[Gelb 79] Arthur Gelb (editor). Applied Optimal Estimation.

MIT Press, Cambridge, Massachusetts, 1979.

[Hildebrand 76] Francis B. Hildebrand.

Advanced Calculus for Applications, Second Ed.

Prentice-Hall, Inc., Englewood Cliffs, New Jersey, 1976.

[Ioannides 79] A. C. Ioannides, G. J. Rogers, and V. Latham.

Stability Limits of a Smith Controller in Simple Systems Containing a Time Delay.

Int. J. Control 29(4):557-563, 1979.

[Ljung 87] Lennart Ljung.

System Identification: Theory for the User.

Prentice-Hall, Inc., Englewood Cliffs, New Jersey, 1987.

[Press 87] William H. Press, Brian P. Flannery, Saul A. Teukolsky, and William

T. Vetterling.

Numerical Recipes: The Art of Scientific Computing.

Cambridge University Press, New York, 1987.

[Sage 71] Andrew P. Sage and James L. Melsa.

System Identification.

Academic Press, New York, 1971.

[Slotine 89] Jean-Jacques E. Slotine, Massachusetts Institute of Technology.

Putting Physics in Control: The Example in Robotics.

1989.

To be published in the I.E.E.E. Control Systems Magazine.

[The Math Works 88]

PC-MATLAB Software.

1988

The MathWorks, Inc., South Natick, Massachusetts.

[Triantafyllou 86] M. S. Triantafyllou, A. Bliek, J. Burgess, and H. Shin. Mooring Dynamics for Offshore Applications, Part 1,.

Technical Report NA81AA-D00069, Sea Grant College Program, MIT, Cambridge, Massachusetts, 1986.

[Triantafyllou 87] M. S. Triantafyllou, Massachusetts Institute of Technology. Notes for Cable Dynamics: WHOI.

April 1987. 